\title{
ON THE THEORY OF ANISOTROPIC FLAT ELASTICITY
}

\author{
A. P. Soldatov
}

UDC 517.9

\begin{abstract}
For the Lamé system from the flat anisotropic theory of elasticity, we introduce generalized double-layer potentials in connection with the function-theory approach. These potentials are built both for the translation vector (the solution of the Lamé system) and for the adjoint vector functions describing the stress tensor. The integral representation of these solutions is obtained using the potentials. As a corollary, the first and the second boundary-value problems in various spaces (Hölder, Hardy, and the class of functions just continuous in a closed domain) are reduced to the equivalent system of the Fredholm boundary equations in corresponding spaces. Note that such an approach was developed in $[19,20]$ for common second-order elliptic systems with constant (higher-order only) coefficients. However, due to important applications, it makes sense to consider this approach in detail directly for the Lamé system. To illustrate these results, in the last two sections we consider the Dirichlet problem with piecewise-constant Lamé coefficients when contact conditions are given on the boundary between two media. This problem is reduced to the equivalent system of the Fredholm boundary equations. The smoothness of kernels of the obtained integral operators is investigated in detail depending on the smoothness of the boundary contours.
\end{abstract}

\section{CONTENTS}

1. Lamé System . . . . . . . . . . . . . . . . . . . . . . . . . . . . . . 484

2. Representation of Solutions of the Lamé System . . . . . . . . . . . . . . . . . . . 490

3. Analytic Functions According to Douglis . . . . . . . . . . . . . . . . . . . . 497

4. The Riemann-Hilbert Problem . . . . . . . . . . . . . . . . . . . . . . . 504

5. First and Second Boundary-Value Problem for the Lamé System . . . . . . . . . . . . 509

6. Double Layer Potentials . . . . . . . . . . . . . . . . . . . . . . . . . . . 513

7. Integral Representations of Double Layer Potentials _ . . . . . . . . . . . . . . 517

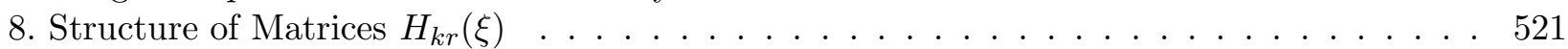

9. Dirichlet Problem in a Piecewise Homogeneous Medium . . . . . . . . . . . . . . . . 526

10. Regularity of Matrix Kernels of Integral Operators . . . . . . . . . . . . . . . . . 530

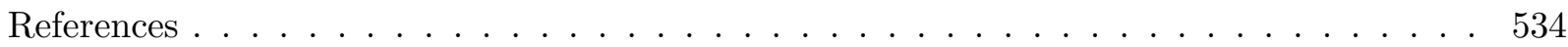

\section{Lamé System}

Consider the Lamé system $[10,11]$

$$
a_{11} \frac{\partial^{2} u}{\partial x^{2}}+\left(a_{12}+a_{21}\right) \frac{\partial^{2} u}{\partial y \partial y}+a_{22} \frac{\partial^{2} u}{\partial y^{2}}=0
$$

Translated from Sovremennaya Matematika. Fundamental'nye Napravleniya (Contemporary Mathematics. Fundamental Directions), Vol. 60, Proceedings of the Seventh International Conference on Differential and Functional Differential Equations and International Workshop "Spatio-Temporal Dynamical Systems" (Moscow, Russia, 22-29 August, 2014). Part 3, 2016. 
with constant coefficients

$$
\begin{array}{ll}
a_{11}=\left(\begin{array}{ll}
\alpha_{1} & \alpha_{6} \\
\alpha_{6} & \alpha_{3}
\end{array}\right), & a_{12}=\left(\begin{array}{ll}
\alpha_{6} & \alpha_{4} \\
\alpha_{3} & \alpha_{5}
\end{array}\right), \\
a_{21}=\left(\begin{array}{ll}
\alpha_{6} & \alpha_{3} \\
\alpha_{4} & \alpha_{5}
\end{array}\right), & a_{22}=\left(\begin{array}{ll}
\alpha_{3} & \alpha_{5} \\
\alpha_{5} & \alpha_{2}
\end{array}\right) .
\end{array}
$$

The elements $\alpha_{j}$ of matrix coefficients, called elasticity moduli, obey the requirement of positive definiteness of the matrix

$$
\alpha=\left(\begin{array}{lll}
\alpha_{1} & \alpha_{4} & \alpha_{6} \\
\alpha_{4} & \alpha_{2} & \alpha_{5} \\
\alpha_{6} & \alpha_{5} & \alpha_{3}
\end{array}\right)
$$

It is convenient to introduce in addition to this matrix its adjugate $\beta=\alpha^{*}$, written in the same form:

$$
\beta=\left(\begin{array}{ccc}
\beta_{1} & \beta_{4} & \beta_{6} \\
\beta_{4} & \beta_{2} & \beta_{5} \\
\beta_{6} & \beta_{5} & \beta_{3}
\end{array}\right), \quad \begin{array}{cc}
\beta_{1}=\alpha_{2} \alpha_{3}-\alpha_{5}^{2} & \beta_{2}=\alpha_{1} \alpha_{3}-\alpha_{6}^{2}, \\
\beta_{3}=\alpha_{1} \alpha_{2}-\alpha_{4}^{2} & \beta_{4}=\alpha_{5} \alpha_{6}-\alpha_{3} \alpha_{4}, \\
\beta_{5}=\alpha_{4} \alpha_{6}-\alpha_{1} \alpha_{5}, & \beta_{6}=\alpha_{4} \alpha_{5}-\alpha_{2} \alpha_{6} .
\end{array}
$$

Then the Sylvester criterium of positive definiteness of the matrix $\alpha$ can be expressed by the inequalities $\operatorname{det} \alpha>0$ and $\alpha_{i}>0, \beta_{i}>0,1 \leq i \leq 3$.

We form a block matrix $a=\left(a_{i j}\right)_{1}^{2}$ from the Lamé coefficients. Then it can be directly seen from (1.2) that for any $\eta \in \mathbb{R}^{4}$ the following equality holds:

$$
(a \eta) \eta=(\alpha \widetilde{\eta}) \widetilde{\eta}, \quad \widetilde{\eta}=\left(\eta_{1}, \eta_{4}, \eta_{2}+\eta_{3}\right),
$$

with respect to scalar products of vectors in $\mathbb{R}^{4}$ and $\mathbb{R}^{3}$. In particular, for any nonzero $\lambda=\left(\lambda_{1}, \lambda_{2}\right) \in \mathbb{R}^{2}$ and $\xi \in \mathbb{R}^{2}$ forming the vector $\eta=\left(\lambda_{1} \xi, \lambda_{2} \xi\right)$ we have the relation

$$
\left[\left(\sum_{i, j=1}^{2} a_{i j} \lambda_{i} \lambda_{j}\right) \xi\right] \xi=(\alpha \widetilde{\eta}) \widetilde{\eta}
$$

Thus vanishing of the left-hand side implies $\widetilde{\eta}=0$ or, equivalently, $\xi=0$. Hence the matrix on the left-hand side of this expression is positive definite:

$$
\sum_{i, j=1}^{2} a_{i j} \lambda_{i} \lambda_{j}>0
$$

and therefore the Lamé system (1.1) is strongly elliptic.

Consider the matrix trinomial $p(z)=a_{11}+\left(a_{12}+a_{21}\right) z+a_{22} z^{2}$ of system (1.1), which is a symmetric matrix

$$
p=\left(\begin{array}{ll}
p_{1} & p_{3} \\
p_{3} & p_{2}
\end{array}\right), \begin{aligned}
& p_{1}(z)=\alpha_{1}+2 \alpha_{6} z+\alpha_{3} z^{2} \\
& p_{2}(z)=\alpha_{3}+2 \alpha_{5} z+\alpha_{2} z^{2} \\
& p_{3}(z)=\alpha_{6}+\left(\alpha_{3}+\alpha_{4}\right) z+\alpha_{5} z^{2} .
\end{aligned}
$$

By (1.5) the determinant det $p(t)>0$ for $t \in \mathbb{R}$, i.e., the characteristic polynomial of the fourth order $\chi=\operatorname{det} p(z)$ of the Lamé system has no real roots. Accordingly, in the upper half-plane it has two roots $\nu_{1}, \nu_{2}$, such that two cases are possible, where $(i) \nu_{1} \neq \nu_{2}$ and $(i i) \nu_{1}=\nu_{2}=\nu$. In the sequel the main role belongs not to these roots as such, but to their symmetric combinations

$$
s=\nu_{1}+\nu_{2}, \quad t=\nu_{1} \nu_{2} .
$$

In the case $(i i)$ of multiple roots they take values $s=2 \nu$ and $t=\nu^{2}$.

With notation $(1.3),(1.6)$ the characteristic polynomial $\chi=p_{1} p_{2}-p_{3}^{2}$ can be written in the form

$$
\chi(z)=\beta_{2}-2 \beta_{5} z+\left(\beta_{3}+2 \beta_{4}\right) z^{2}-2 \beta_{6} z^{3}+\beta_{1} z^{4} .
$$


It is not difficult to describe the conditions on its coefficients that ensure the case $(i i)$ of multiple roots. In this case one must have

$$
\chi(z)=\beta_{1}(z-\nu)^{2}(z-\bar{\nu})^{2}, \quad(z-\nu)(z-\bar{\nu})=\delta_{0}+\delta_{1} z+z^{2},
$$

where $\delta_{1}^{2}<4 \delta_{0}$. Equalizing the coefficients at the same powers of $z$, we obtain the equations

$$
\delta_{0}=\frac{\beta_{2}}{\beta_{1}}, \quad \delta_{1}=-\frac{\beta_{6}}{\beta_{1}}=-\frac{\beta_{5}}{\sqrt{\beta_{1} \beta_{2}}}
$$

for coefficients $\delta_{j}$ and relations

$$
\beta_{5}^{2}<4 \beta_{2}^{2}, \quad \beta_{5}=\beta_{6} \sqrt{\beta_{2} / \beta_{1}}, \quad \beta_{5}^{2}+2 \beta_{2}^{2}=\beta_{2}\left(\beta_{3}+2 \beta_{4}\right),
$$

that are necessary and sufficient for the multiplicity of the roots of the characteristic polynomial. In this case according to (1.9) we have the expression

$$
2 \beta_{1} \nu=\beta_{6}+i \sqrt{4 \beta_{1} \sqrt{\beta_{1} \beta_{2}}-\beta_{6}^{2}}
$$

for the root $\nu$.

In the class $\mathcal{A}$ of positive definite matrices of the form (1.2) we define subsets $\mathcal{A}_{1}$ and $\mathcal{A}_{2}$, by the condition of linear independence of the polynomials $p_{2}, p_{3}$ and $p_{1}, p_{3}$ that appear in (1.6), respectively. Evidently, the complements of these subsets can be described by the conditions

$$
\begin{gathered}
\alpha \notin \mathcal{A}_{1} \Leftrightarrow \alpha_{3} \alpha_{5}=\alpha_{2} \alpha_{6}, \alpha_{2}\left(\alpha_{3}+\alpha_{4}\right)=2 \alpha_{5}^{2} \Leftrightarrow \chi(\nu)=p_{2}(\nu)=0, \\
\alpha \notin \mathcal{A}_{2} \Leftrightarrow \alpha_{1} \alpha_{5}=\alpha_{3} \alpha_{6}, \alpha_{1}\left(\alpha_{3}+\alpha_{4}\right)=2 \alpha_{6}^{2} \Leftrightarrow \chi(\nu)=p_{1}(\nu)=0
\end{gathered}
$$

for one of the roots $\nu$ of the characteristic equation. The second equivalence follows from the fact that $\chi=p_{1} p_{2}-p_{3}^{2}$. Therefore equalities $p_{j}(\nu)=p_{3}(\nu)=0$ for one of the values $j=1,2$ are equivalent to the linear dependence of the polynomials $p_{j}$ and $p_{3}$.

Note that

$$
\alpha_{3}^{2}<\alpha_{1} \alpha_{2} \text { for } \alpha \notin \mathcal{A}_{1} \cap \mathcal{A}_{2} \text {. }
$$

In fact, let, e.g., the conditions $\alpha \notin \mathcal{A}_{1}$ in (1.11) hold. Then $2 \alpha_{2} \alpha_{6}^{2}=\alpha_{3}^{2}\left(\alpha_{3}+\alpha_{4}\right), 2 \alpha_{1} \alpha_{5}^{2}=$ $\alpha_{1} \alpha_{2}\left(\alpha_{3}+\alpha_{4}\right)$. Substituting these expressions into the formula of the determinant of the matrix (1.2), we get

$$
2 \operatorname{det} \alpha=\left(\alpha_{1} \alpha_{2}-\alpha_{3}^{2}\right)\left(\alpha_{3}-\alpha_{4}\right)>0 \text {. }
$$

It remains to note that the inequalities $\alpha_{1} \alpha_{2}-\alpha_{3}^{2}<0$ and $\alpha_{3}<\alpha_{4}$ contradict the inequality $\alpha_{1} \alpha_{2}-\alpha_{4}^{2}>0$. The case $\alpha \notin \mathcal{A}_{2}$ is considered in a similar way.

We also introduce the class $\mathcal{A}_{0}$ of matrices $\alpha$, for which $p_{3}=0$, i.e., $\alpha_{3}+\alpha_{4}=\alpha_{5}=\alpha_{6}=0$. Obviously, this class does not intersect the sets $\mathcal{A}_{1}$ and $\mathcal{A}_{2}$. From (1.12) it easily follows that

$$
\mathcal{A}=\mathcal{A}_{0} \cup \mathcal{A}_{1} \cup \mathcal{A}_{2} \text {. }
$$

In fact, let $\alpha \notin \mathcal{A}_{1} \cup \mathcal{A}_{2}$, so that the respective conditions in (1.11) hold simultaneously. Then the first equalities (1.11) imply $\alpha_{5} \alpha_{6}\left(\alpha_{1} \alpha_{2}-\alpha_{3}^{2}\right)=0$, so that due to (1.12) one must have $\alpha_{5} \alpha_{6}=0$. By (1.11), this implies $\alpha \in \mathcal{A}_{0}$.

Similar arguments show that the matrix $p(\nu)$ is distinct from the zero one for all $\nu$ :

$$
p(\nu) \neq 0, \quad \nu \in \mathbb{C} .
$$

In fact, if $p(\nu)=0$, then $\operatorname{Im} \nu \neq 0$ and $\alpha \notin \mathcal{A}_{j}$ for both values $j=1,2$. Therefore $\alpha \in \mathcal{A}_{0}$, but in this case the polynomials $p_{1}$ and $p_{2}$ are linearly independent.

In the cases where $\alpha$ belongs to one of the exceptional sets $\mathcal{A}_{0}$ and $\mathcal{A} \backslash \mathcal{A}_{j}, \quad j=1,2$, the roots $\nu_{j}$ of the characteristic equation can be calculated explicitly by means of quadratic equations.

For $\alpha \in \mathcal{A}_{0}$ the Lamé system becomes diagonal, i.e., it dissolves into two equations

$$
\alpha_{1} \frac{\partial^{2} u_{1}}{\partial x^{2}}+\alpha_{3} \frac{\partial^{2} u_{1}}{\partial y^{2}}=0, \quad \alpha_{3} \frac{\partial^{2} u_{2}}{\partial x^{2}}+\alpha_{2} \frac{\partial^{2} u_{2}}{\partial y^{2}}=0
$$


In this case $\chi=p_{1} p_{2}$ and one can consider $p_{j}\left(\nu_{j}\right)=0$ or, in the explicit form,

$$
\nu_{1}=i \sqrt{\frac{\alpha_{1}}{\alpha_{3}}}, \quad \nu_{2}=i \sqrt{\frac{\alpha_{3}}{\alpha_{2}}} .
$$

In the case $\alpha \notin \mathcal{A}_{j}$ the roots of the characteristic equation can be found from the equations

$$
\begin{array}{ll}
\left(\alpha_{2}^{2} p_{1}-\alpha_{5}^{2} p_{2}\right)\left(\nu_{1}\right)=0, p_{2}\left(\nu_{2}\right)=0, & \alpha \notin \mathcal{A}_{1} ; \\
p_{1}\left(\nu_{1}\right)=0,\left(\alpha_{3}^{2} p_{2}-\alpha_{5}^{2} p_{1}\right)\left(\nu_{2}\right)=0, & \alpha \notin \mathcal{A}_{2} .
\end{array}
$$

In fact, let, for example, $\alpha \notin \mathcal{A}_{1}$. Then $\alpha_{2} p_{3}-\alpha_{5} p_{2}=0$ and we have the equality $\alpha_{2}^{2} \chi=\left(\alpha_{2}^{2} p_{1}-\right.$ $\left.\alpha_{5}^{2} p_{2}\right) p_{2}$.

The case $\alpha \notin \mathcal{A}_{2}$ ca be considered in a similar way. Note that the numbering of roots used in (1.16) agrees with the case $\alpha \in \mathcal{A}_{0}$ in (1.15).

In all three mentioned cases the roots $\nu_{j}$ are distinct. In the case (1.15) this fact follows from (1.12). If these roots coincide in case (1.16), then $p_{1}(\nu)=p_{2}(\nu)=0$ and hence $p(\nu)=0$, which contradicts (1.14). In particular, in the situation with a multiple root the matrix $\alpha$ necessarily belongs to $\mathcal{A}_{1} \cap \mathcal{A}_{2}$.

Let us agree to call the nonzero vector $x \in \mathbb{C}^{2}$ an eigenvector of the polynomial $p$ corresponding to the root $\nu$ the characteristic equation if $p(\nu) x=0$ and an adjoined vector if $p(\nu) x+p^{\prime}(\nu) y=0$, where $p(\nu) y=0$ and $p^{\prime}(\nu) y \neq 0$.

\section{Lemma 1.1.}

(a) Let the roots $\nu_{j}$ be distinct. Then there exists a base $e=\left\{e_{1}, e_{2}\right\}$ of the space $\mathbb{C}^{2}$ consisting of eigenvectors, e.g., $p\left(\nu_{j}\right) e_{j}=0, j=1,2$. Any other base $\tilde{e}$ of this type is linked with $e$ by the relation $\tilde{e}_{j}=\lambda_{j} e_{j}, j=1,2$.

(b) Let the root $\nu$ be multiple. Then there exists a base $e=\left\{e_{1}, e_{2}\right\}$ consisting of an eigenvector and an adjoined vector, i.e.,

$$
p(\nu) e_{1}=0, \quad p(\nu) e_{2}+p^{\prime}(\nu) e_{1}=0 .
$$

Any other base $\tilde{e}$ of this type is linked with e by the relation $\tilde{e}_{1}=\lambda e_{2}, \tilde{e}_{2}=\lambda e_{2}+\lambda_{0} e_{1}, \lambda \neq 0$. Proof.

(a) By (1.14) the spaces $\left\{x \in \mathbb{C}^{2}, p\left(\nu_{j}\right) x=0\right\}, j=1,2$, are one-dimensional; therefore it suffices to check that for a nonzero vector $x \in \mathbb{C}^{2}$ the simultaneous equalities $p\left(\nu_{1}\right) x=p\left(\nu_{2}\right) x=0$ are impossible.

In fact, suppose that such a vector $x=\left(x_{1}, x_{2}\right)$ does exist. Then necessarily $x_{1} x_{2} \neq 0$, since the polynomials $p_{1}$ and $p_{2}$ cannot have both numbers $\nu_{j}$ as their roots. Thus one can consider that $x_{1}=1, x_{2}=\lambda$. Then the inequality $p\left(\nu_{j}\right) x=0$ reduces to two scalar equalities $\left(p_{1}+\lambda p_{3}\right)\left(\nu_{j}\right)=0$, $\left(p_{3}+\lambda p_{2}\right)\left(\nu_{j}\right)=0$. Therefore the polynomials $p_{1}+\lambda p_{3}$ and $p_{3}+\lambda p_{2}$ have the polynomial $q(z)=$ $\left(z-\nu_{1}\right)\left(z-\nu_{2}\right)$ as a divisor, so that

$$
p_{1}+\lambda p_{3}=c_{1} q, \quad p_{3}+\lambda p_{2}=c_{2} q
$$

with some factors $c_{j} \in \mathbb{C}$. Since the coefficients of the polynomials $p_{j}$ are real, hence we have

$$
p_{1}+\bar{\lambda} p_{3}=\bar{c}_{1} \bar{q}, \quad p_{3}+\bar{\lambda} p_{2}=\bar{c}_{2} \bar{q},
$$

where $\bar{q}(z)=\left(z-\bar{\nu}_{1}\right)\left(z-\bar{\nu}_{2}\right)$. Since a quadratic trinomial with real coefficients cannot be a multiple of $q$, the number $\lambda$ is not real. Therefore from these systems one can express the polynomials $p_{j}$ as a function of $q, \bar{q}$ by the formulas $p_{j}=d_{j} q+\bar{d}_{j} \bar{q}, 1 \leq j \leq 3$, with respective coefficients $d_{j} \in \mathbb{C}$. In particular,

$$
p_{1} p_{2}-p_{3}^{2}=\left(d_{1} d_{2}-d_{3}^{2}\right) q^{2}+\left(d_{1} \bar{d}_{2}+\bar{d}_{1} d_{2}-2 d_{3} \bar{d}_{3}\right) q \bar{q}+\left(\bar{d}_{1} \bar{d}_{2}-\bar{d}_{3}^{2}\right) \bar{q}^{2}
$$

On he other hand, by (1.6) in our notation

$$
p_{1} p_{2}-p_{3}^{2}=c q \bar{q}, \quad c=\alpha_{2} \alpha_{3}-\alpha_{5}^{2}>0 .
$$


Hence one must have $d_{1} d_{2}-d_{3}^{2}=0$ and $2 \operatorname{Re}\left(d_{1} \bar{d}_{2}\right)-2 d_{3} \bar{d}_{3}=c$ (otherwise the polynomial $\bar{q}^{2}$ has $q$ as a divisor, which is impossible). But then $c=2\left[\operatorname{Re}\left(d_{1} \bar{d}_{2}\right)-\left|d_{1} \bar{d}_{2}\right|\right]$, which contradict the inequality $c>0$.

(b) First of all, show that the simultaneous equalities $p(\nu) x=p^{\prime}(\nu) x=0$ are impossible for any nonzero vector $x \in \mathbb{C}$. In fact, suppose that such a vector $x=\left(x_{1}, x_{2}\right)$ does exist. Then necessarily $x_{1} x_{2} \neq 0$, since for real polynomials $p_{j}$ the equalities $p_{j}(\nu)=p_{j}^{\prime}(\nu)=0$ are impossible. As in the case of distinct roots, we arrive at the relations

$$
p_{1}+\lambda p_{3}=c_{1} q, \quad p_{3}+\lambda p_{2}=c_{2} q,
$$

where $q(z)=(z-\nu)^{2}$. The further arguments are similar to those from the proof of item a).

Thus any pair of nonzero vectors $e_{1}, e_{2}$ with property (1.17) is a base. The existence of such a pair will be shown below in Lemma 1.2.

It remains to establish the connection between two bases $e$ and $\tilde{e}$ that satisfy (1.17). Obviously, $\tilde{e}_{1}=\lambda e_{1}, \lambda \neq 0$, and $\tilde{e}_{2}=\lambda_{0} e_{1}+\lambda^{\prime} e_{2}, \lambda^{\prime} \neq 0$. Therefore

$$
p(\nu) \tilde{e}_{2}+p^{\prime}(\nu) \tilde{e}_{1}=\lambda^{\prime} p(\nu) e_{2}+\lambda p^{\prime}(\nu) e_{1}=0 .
$$

Subtracting form this equality the second equality (1.17) multiplied by $\lambda^{\prime}$, we obtain $\left(\lambda-\lambda^{\prime}\right) p^{\prime}(\nu) e_{1}=0$, hence, $\lambda^{\prime}=\lambda$.

Consider the adjoined matrix of $p$

$$
p^{*}=\left(\begin{array}{cc}
p_{2} & -p_{3} \\
-p_{3} & p_{1}
\end{array}\right)
$$

\section{Lemma 1.2.}

(i) In the case of distinct roots for $\alpha \in \mathcal{A}_{k}, k=1,2$, the condition $p\left(\nu_{j}\right) e_{j}=0$ of proposition a) of Lemma 1.1 is satisfied by the $k$-th column $e_{j}=p_{(k)}^{*}\left(\nu_{j}\right)$. If $\alpha \notin \mathcal{A}_{1}$, then in notation (1.16) one can put $e_{j}=p_{(j)}^{*}\left(\nu_{j}\right)$. Respectively, in the case $\alpha \notin \mathcal{A}_{2}$ one can similarly put $e_{1}=p_{(1)}^{*}\left(\nu_{2}\right)$, $e_{2}=p_{(2)}^{*}\left(\nu_{1}\right)$.

(ii) In the case of a multiple root the matrix $\alpha \in \mathcal{A}_{1} \cap \mathcal{A}_{2}$ and the condition (1.17) is satisfied by the vectors $e_{1}=p_{(k)}^{*}(\nu)$ and $e_{2}=\left(p^{*}\right)_{(k)}^{\prime}(\nu)$ for any value $k=1,2$, where the prime denotes the derivative of the matrix (1.18).

Proof. Prove the statement for each of the cases $(i)$ and (ii) separately.

$(i)$. We make use of the obvious relation

$$
p(z) p^{*}(z)=\chi(z)
$$

where $\chi$ on the right-hand side denotes a scalar matrix. This relation implies that $p\left(\nu_{j}\right) p_{(k)}^{*}\left(\nu_{j}\right)=0$ for any value $k=1,2$. In other words, if the column $p_{(k)}^{*}\left(\nu_{j}\right)$ of the matrix $p^{*}\left(\nu_{j}\right)$ is nonzero, then it is an eigenvector corresponding to $\nu_{j}$. By (1.18), for $k=1(k=2)$ it can be zero only if $\alpha \notin \mathcal{A}_{1}$ ( $\alpha \notin \mathcal{A}_{2}$ ). Hence, taking into account Lemma 1.1, we immediately obtain the first assertion of the lemma.

(ii). Since by hypothesis $\chi(\nu)=\chi^{\prime}(\nu)=0$, we have

$$
p(\nu) p^{*}(\nu)=p(\nu)\left(p^{*}\right)^{\prime}(\nu)+p^{\prime}(\nu) p^{*}(\nu)=0 .
$$

Therefore the vectors $e_{1}=p_{(k)}^{*}(\nu)$ and $e_{2}=\left(p^{*}\right)_{(k)}^{\prime}(\nu)$ satisfy (1.17). Since, as we have noted before, in the considered case of the multiple root $\nu$ the matrix $\alpha$ belongs to $\mathcal{A}_{1} \cap \mathcal{A}_{2}$, these vectors are nonzero.

An elastic medium is called orthotropic if

$$
\alpha_{5}=\alpha_{6}=0 .
$$


In this case the coordinate axes are the axes of symmetry of the elastic medium, and we have simpler expressions

$$
p_{1}(z)=\alpha_{1}+\alpha_{3} z^{2}, p_{2}(z)=\alpha_{3}+\alpha_{2} z^{2}, p_{3}(z)=\left(\alpha_{3}+\alpha_{4}\right) z
$$

for polynomials (1.6). In particular, in an orthotropic medium one has either $\alpha \in \mathcal{A}_{1} \cap \mathcal{A}_{2}$ or $\alpha \in \mathcal{A}_{0}$.

From (1.21) it follows that the characteristic equation $p_{1} p_{2}-p_{3}^{2}=0$ is biquadratic and its roots $\nu$ in the upper half-plane can be expressed explicitly. For this purpose we introduce positive quantities $\rho$ and $\rho_{0}$ by the formulas

$$
\rho^{2}=\sqrt{\frac{\alpha_{1}}{\alpha_{2}}}, \quad \rho_{0}^{2}=\frac{\alpha_{1} \alpha_{2}-\alpha_{4}^{2}+2 \alpha_{3}\left(\sqrt{\alpha_{1} \alpha_{2}}-\alpha_{4}\right)}{\alpha_{2} \alpha_{3}} .
$$

The positivity of the expression on the right-hand side of the second equality follows from the condition $\alpha_{4}^{2}<\alpha_{1} \alpha_{2}$. For the same reason, the quantity

$$
\rho_{0}^{2}-4 \rho^{2}=\frac{\left(\sqrt{\alpha_{1} \alpha_{2}}-\alpha_{4}\right)\left(\sqrt{\alpha_{1} \alpha_{2}}-\alpha_{4}-2 \alpha_{3}\right)}{\alpha_{2} \alpha_{3}}
$$

has the same sign as $\sqrt{\alpha_{1} \alpha_{2}}-\alpha_{4}-2 \alpha_{3}$.

In this notation for the roots $\nu$ we have formulas

$$
\begin{gathered}
\nu_{1,2}=i \rho e^{ \pm i \theta}, \quad 2 \theta=\arccos \left[\frac{\rho_{0}^{2}-2 \rho^{2}}{2 \rho^{2}}\right], \text { if } \rho_{0}<2 \rho, \\
\nu_{1,2}=i \rho e^{ \pm \tau}, \quad 2 \tau=\operatorname{arcch}\left[\frac{\rho_{0}^{2}-2 \rho^{2}}{2 \rho^{2}}\right], \text { if } \rho_{0}>2 \rho, \\
\nu_{1}=\nu_{2}=i \rho, \text { if } \rho_{0}=2 \rho .
\end{gathered}
$$

In fact, let $\delta$ denote the expression in square brackets $(1.24)$, so that $\rho_{0}^{2}=2(\delta+1) \rho^{2}$. Then

$$
p_{1}(z) p_{2}(z)-p_{3}^{2}(z)=\alpha_{2} \alpha_{3}\left(\rho^{4}+2 \delta \rho^{2} z^{2}+z^{4}\right) .
$$

Therefore, $\nu^{2}=-\rho^{2}\left(\delta \pm \sqrt{\delta^{2}-1}\right)$, which after elementary transformations results in (1.24).

Note that the case $\alpha \in \mathcal{A}_{0}$ of a diagonalizable Lamé system corresponds to the first equality in (1.20), and in this case the expressions for the roots $\nu_{j}$ coincide with (1.14). The case $(i i)$ of multiple roots corresponds to the last equality in (1.24). Note that this fact agrees with the criterion (1.9), (1.10) of multiple roots.

It is easy to see that independently of the three possible cases in (1.24) we have the same expressions

$$
s=i \rho_{0}, t=-\rho^{2}
$$

for the sum and product (1.7) of the roots.

An orthotropic medium is called isotropic if, in addition to (1.20), the following relations hold:

$$
\alpha_{1}=\alpha_{2}=2 \alpha_{3}+\alpha_{4} \text {. }
$$

Together with the inequality $\alpha_{4}^{2}<\alpha_{1} \alpha_{2}$ this implies that $\alpha_{1}>\alpha_{3}$, so that the quantity

$$
\varkappa=\frac{\alpha_{1}+\alpha_{3}}{\alpha_{1}-\alpha_{3}}>1
$$

It is easy to see that in the case under consideration the characteristic equation has a multiple root $\nu=i$. 


\section{Representation of Solutions of the Lamé System}

In accordance with two cases $(i)$ and $(i i)$ of the roots of the characteristic equation introduce the matrices

$$
\text { (i) } J=\left(\begin{array}{cc}
\nu_{1} & 0 \\
0 & \nu_{2}
\end{array}\right), \quad(\text { ii }) J=\left(\begin{array}{cc}
\nu & 1 \\
0 & \nu
\end{array}\right) .
$$

We relate to this matrix an elliptic system of the first order of the special form

$$
\frac{\partial \phi}{\partial y}-J \frac{\partial \phi}{\partial x}=0
$$

for $J=i$ it corresponds to the Cauchy-Riemann system, which defines analytic functions. For this reason, the solutions $\phi=\left(\phi_{1}, \phi_{2}\right)$ of this system are called $J$-analytic functions.

In the studies of boundary value problems of flat elasticity by classical methods one can find two main trends. The first one consists in using analytic functions similarly to Kolosov-Muskhelishvili formulas [12] in the isotropic case. This trend is present in the papers by Lekhnitsky, Savin, Mikhlin, etc. (see, e.g., $[5,11,12])$. The other one consists in application of solutions of some elliptic systems of the first order instead of analytic functions (see, e.g., $[2,3,7]$ ). The approach considered below is adjacent to this trend and based on system (2.2), more exactly, on the representation of the general solution of the Lamé system by $J$-analytic functions. This representation itself is based on the following structural lemma.

Lemma 2.1. There exists an invertible matrix $b \in \mathbb{C}^{2 \times 2}$ such that

$$
a_{11} b+\left(a_{12}+a_{21}\right) b J+a_{22} b J^{2}=0,
$$

and any other matrix $\tilde{b}$ with the same properties is related to $b$ by the equality $\tilde{b}=b d$ with some invertible matrix d that commutes with $J$.

Equality (2.3) is equivalent to the relation

$$
A B=B \operatorname{diag}(J, \bar{J})
$$

for block matrices

$$
A=\left(\begin{array}{cc}
0 & 1 \\
a_{0} & a_{1}
\end{array}\right), \quad B=\left(\begin{array}{cc}
b & \bar{b} \\
b J & \overline{b J}
\end{array}\right)
$$

where we put $a_{0}=-a_{22}^{-1} a_{11}, a_{1}=-a_{22}^{-1}\left(a_{12}+a_{21}\right)$. Here the matrix $B$ is invertible.

Proof. From (2.1) one can see that for any matrix $b \in \mathbb{C}^{2 \times 2}$ in case $(i)$ of distinct roots for the $j$-th column we have relations $(b J)_{(j)}=\nu_{j} b_{(j)},\left(b J^{2}\right)_{(j)}=\nu_{j}^{2} b_{(j)}$ and therefore

$$
\left(a_{11}+\left(a_{12}+a_{21}\right) b J+a_{22} b J^{2}\right)_{(j)}=p\left(\nu_{j}\right) b_{(j)} \quad(j=1,2) .
$$

Similarly in case $(i i)$ of a multiple root

$$
\begin{aligned}
(b J)_{(1)} & =\nu b_{(1)}, \quad(b J)_{(2)}=b_{(1)}+\nu b_{(2)}, \\
\left(b J^{2}\right)_{(1)} & =\nu^{2} b_{(1)}, \quad\left(b J^{2}\right)_{(2)}=2 \nu b_{(1)}+\nu^{2} b_{(2)},
\end{aligned}
$$

whence one has

$$
\begin{gathered}
\left(a_{11}+\left(a_{12}+a_{21}\right) b J+a_{22} b J^{2}\right)_{(1)}=p(\nu) b_{(1)}, \\
\left(a_{11}+\left(a_{12}+a_{21}\right) b J+a_{22} b J^{2}\right)_{(2)}=p(\nu) b_{(2)}+p^{\prime}(\nu) b_{(1)} .
\end{gathered}
$$

Therefore, choosing the vectors $e_{j}$ of Lemma 1.1 as columns $b_{(j)}$, we arrive at the validity of the first part of the Lemma. One must just take into consideration that the matrices $d$ that commute with $J$ have the form

$$
\text { (i) } d=\left(\begin{array}{cc}
d_{1} & 0 \\
0 & d_{2}
\end{array}\right), \quad(\text { ii }) J=\left(\begin{array}{cc}
d_{1} & d_{0} \\
0 & d_{1}
\end{array}\right) \text {. }
$$

The equivalence of relations (2.3) and (2.4) is obvious, therefore it remains to establish the invertibility of the matrix $B$. 
Consider the invertible matrix $B_{1}$ that takes $A$ to the Jordan form. Since the matrix $A$ is real, the Jordan matrix can be chosen in the form $\operatorname{diag}\left(J_{1}, \overline{J_{1}}\right)$, where the eigenvalues of the matrix $J_{1}$ lie in the upper half-plane and this matrix is either diagonal or a Jordan block. Accordingly, $B_{1}$ can be reduced to the block structure

$$
B_{1}=\left(\begin{array}{ll}
b_{1} & \overline{b_{1}} \\
b_{2} & \overline{b_{2}}
\end{array}\right)
$$

Thus,

$$
A B_{1}=B_{1} \operatorname{diag}\left(J_{1}, \overline{J_{1}}\right)
$$

Due to the obvious identity

$$
(z-A)\left(\begin{array}{ll}
1 & 1 \\
0 & z
\end{array}\right)=\left(\begin{array}{cc}
z & 0 \\
-a_{0} & z^{2}-a_{1} z-a_{0}
\end{array}\right)
$$

the eigenvalues of the matrix $A$ in the upper half-plane coincide with the roots of the characteristic equation $\operatorname{det} p(z)=0$. Therefore either $J_{1}=J$, or the matrix $J_{1}$ is scalar: $J_{1}=\nu$. We show that the second case is impossible. In fact, if $J_{1}=\nu$, then there exist two linearly independent eigenvectors $\eta, \tilde{\eta} \in \mathbb{C}^{4}$ of the matrix $A$ that correspond to the eigenvalue $\nu$. But then the form (2.5) of this matrix implies that $\eta=\left(\eta_{1}, \eta_{2}\right)$, where the vectors $\eta_{j} \in \mathbb{C}^{2}$ satisfy the relations $\eta_{2}=\nu \eta_{1}, p(\nu) \eta_{1}=0$ and the vector $\tilde{\eta}$ possesses a similar property. But then the vectors $\eta_{1}$ and $\tilde{\eta}_{1}$, and therefore also the vectors $\eta, \tilde{\eta}$ must be linearly dependent, which contradicts our assumption.

Thus, the matrix $J_{1}=J$ and (2.4) implies that $b_{2}=b_{1} J$ and the matrix $b_{1}$ satisfies (2.3). Passing to the columns of these matrices, as in the proof of Lemma 1.1, we see that $b_{1}=b d$ with some matrix $d$ that commutes with $J$. But then $B_{1}=B \operatorname{diag}(d, \bar{d})$, whence $\operatorname{det} B_{1}=\operatorname{det} B|\operatorname{det} d|^{2}$. Therefore the matrices $B$ and $d$ are invertible, which completes the proof of the Lemma.

We turn to system (2.2) in some domain $D$ of the complex plain. We will denote the partial derivatives of its solutions as

$$
\phi^{\prime}=\frac{\partial \phi}{\partial x}, \quad J \phi^{\prime}=\frac{\partial \phi}{\partial y}
$$

Then if we relate the matrix

$$
z_{J}=x+y J
$$

to the complex number $z=x+i y$, we can write

$$
\frac{\partial \phi}{\partial x} d x+\frac{\partial \phi}{\partial y} d y=d z_{J} \phi^{\prime}
$$

In particular, the function $\phi$ can be restored from its derivative $\psi=\phi^{\prime}$ by the curvilinear integral

$$
\phi(z)=\phi\left(z_{0}\right)+\int_{z_{0}}^{z} d t_{J} \psi(t)
$$

along some smooth arc joining the points $z$ and $z_{0}$ in the domain $D$.

Conversely, if a $J$-analytic function $\psi$ is given, this formula defines a $J$-analytic function $\phi$ such that its derivative coincides with $\psi$. If the domain $D$ is simply connected, this formula defines a singlevalued function. But in the case of a multiply connected domain, generally speaking, $\phi$ is multivalued and can admit nonzero increments while tracing the connected components of the boundary $\partial D$. In the sequel we understand under multivalued functions such ones, which have single-valued derivatives.

Theorem 2.1. In the notation of Lemma 2.1 any solution $u$ of the Lamé system (1.1) in the domain $D$ can be represented in the form

$$
u=\operatorname{Re} b \phi
$$


with some (generally multivalued) J-analytic function $\phi$ in this domain, and its derivative $\phi^{\prime}$ can be restored from the gradient grad $u$ of this solution by the formula

$$
\phi^{\prime}=2\left(d_{1} \frac{\partial u}{\partial x}+d_{2} \frac{\partial u}{\partial y}\right), \quad\left(\frac{d_{1}}{d_{1}} \frac{d_{2}}{d_{2}}\right)=B^{-1} .
$$

Proof. In notation (2.5) with respect to the vector $U=\operatorname{grad} u$ system (1.1) can be written as a first order one

$$
\frac{\partial U}{\partial y}-A \frac{\partial U}{\partial x}=0
$$

By (2.4), with respect to $V=B^{-1} U$ this system passes into

$$
\frac{\partial V}{\partial y}-\operatorname{diag}(J, \bar{J}) \frac{\partial V}{\partial x}=0
$$

Since the vector $U$ is real, the vector $V=B^{-1} \operatorname{grad} u$ has a block structure $(\psi, \bar{\psi})$, so that the function $\psi$ is $J$-analytic and

$$
\operatorname{grad} u=B V, \quad V=(\psi, \bar{\psi}) .
$$

Thus according to the form (2.5) of the matrix $B$ we have

$$
\frac{\partial u}{\partial x}=2 \operatorname{Re} b \psi, \quad \frac{\partial u}{\partial y}=2 \operatorname{Re} b J \psi
$$

Hence, similarly to (2.9), we arrive at the equality

$$
u=\operatorname{Re} b \phi+\xi, \quad \xi \in \mathbb{R}^{2},
$$

where the $J$-analytic function $\phi$ has a derivative $\phi^{\prime}=\psi$. Due to the invertibility of the matrix $B$ there exists a vector $\eta \in \mathbb{C}^{2}$ such that $\operatorname{Re} b \eta=\xi$. Therefore, denoting $\phi+\eta$ by $\phi$ again, we finally arrive at representation (2.10).

As for equality (2.11), it is equivalent to (2.12) with respect to $\psi=\phi^{\prime}$.

Theorem 2.1 can be complemented by a representation formula for the stress tensor

$$
\sigma=\left(\begin{array}{ll}
\sigma_{1} & \sigma_{3} \\
\sigma_{3} & \sigma_{2}
\end{array}\right)
$$

Recall that the vector $u=\left(u_{1}, u_{2}\right)$ characterizes the shift vector; it is related to the columns $\sigma_{(1)}, \sigma_{(2)}$ of the stress tensor by the equalities

$$
\sigma_{(i)}=a_{i 1} \frac{\partial u}{\partial x}+a_{i 2} \frac{\partial u}{\partial y}, i=1,2,
$$

that form the content of Hooke's law.

In the absence of mass forces the matrix $\sigma$ satisfies equilibrium equations

$$
\frac{\partial \sigma_{(1)}}{\partial x}+\frac{\partial \sigma_{(2)}}{\partial y}=0
$$

which together with (2.13) result in the Lamé system.

The columns of the tensor can be conveniently described as the partial derivatives of the so-called adjoint function $v$. The latter is defined by the relation

$$
\frac{\partial v}{\partial x}=-\left(a_{21} \frac{\partial u}{\partial x}+a_{22} \frac{\partial u}{\partial y}\right), \quad \frac{\partial v}{\partial y}=a_{11} \frac{\partial u}{\partial x}+a_{12} \frac{\partial u}{\partial y} .
$$

Writing (1.1) in the form

$$
\frac{\partial}{\partial x}\left(a_{11} \frac{\partial u}{\partial x}+a_{12} \frac{\partial u}{\partial y}\right)+\frac{\partial}{\partial y}\left(a_{21} \frac{\partial u}{\partial x}+a_{22} \frac{\partial u}{\partial y}\right)=0,
$$


we see that the necessary condition of the existence of the function $v$ holds. Therefore up to an additive constant $\xi \in \mathbb{R}^{l}$ it is uniquely defined in each simply connected subdomain $D_{0} \subseteq D$. In the whole domain this function can be multivalued and admit bifurcations while encircling the connected components of the boundary $\partial D$.

If the adjoint function $v$ is constant, then (2.14) is a homogeneous system with respect to the gradient vector $\eta$; therefore, due to (1.4) we have

$$
\frac{\partial u_{1}}{\partial x}=\frac{\partial u_{1}}{\partial y}+\frac{\partial u_{2}}{\partial x}=\frac{\partial u_{2}}{\partial y}=0
$$

identically in the domain $D$. Evidently, these relations are equivalent to

$$
u_{1}(x, y)=\lambda_{1}-\lambda_{0} y, \quad u_{2}(x, y)=\lambda_{1}+\lambda_{0} x
$$

with some $\lambda_{j} \in \mathbb{R}$. We will call solutions of this type trivial, they correspond to translation of the elastic medium as a whole.

From (2.13), (2.14) we reach the expressions

$$
\sigma_{(1)}=\frac{\partial v}{\partial y}, \quad \sigma_{(2)}=-\frac{\partial v}{\partial x}
$$

for the columns of the stress tensor.

Theorem 2.2. Under the hypotheses of Theorem 2.1 the adjoint function of the solution (2.10) of the Lamé system is representable as

$$
v=\operatorname{Re} c \phi, \quad c=-\left(a_{21} b+a_{22} b J\right) .
$$

In this case $v$ is constant if and only if the derivative $\phi^{\prime}$ is constant in this representation.

Proof. Differentiating (2.10) and substituting the result into (2.14), we get

$$
\frac{\partial v}{\partial x}=-\operatorname{Re}\left(a_{21} b+a_{22} b J\right) \phi^{\prime}, \quad \frac{\partial v}{\partial y}=\operatorname{Re}\left(a_{11} b+a_{12} b J\right) \phi^{\prime} .
$$

By (2.11), for the matrix $c$ in $(2.17)$ we have the relation $a_{11} b+a_{12} b J=c J$, so that

$$
\frac{\partial v}{\partial x}=\operatorname{Re} c \phi^{\prime}, \quad \frac{\partial v}{\partial y}=\operatorname{Re} c J \phi^{\prime}
$$

As in the proof of Theorem 2.1, we arrive at (2.17).

If the function $v$ is constant in the representation (2.17), as we have mentioned above, the solution $u$ of the Lamé system is trivial and has the form (2.15). Together with (2.10) this leads to a system

$$
\operatorname{Re} b \phi^{\prime}=\xi, \quad \operatorname{Re} c \phi^{\prime}=0
$$

with a vector $\xi=\left(0, \lambda_{0}\right) \in \mathbb{R}^{2}$. By Lemma 2.1, the matrix

$$
\left(\begin{array}{cc}
b & \bar{b} \\
c & \bar{c}
\end{array}\right)=\left(\begin{array}{cc}
1 & 0 \\
-a_{21} & -a_{22}
\end{array}\right)\left(\begin{array}{cc}
b & \bar{b} \\
b J & \overline{b J}
\end{array}\right)
$$

is invertible, so that the previous system yields a constant vector $\phi^{\prime}$.

By Lemma 1.2 it is easy to give explicit expressions for the matrices $b$ and $c$ mentioned in Theorems 2.1, 2.2. We introduce these expressions separately for each of the three cases where the matrix $\alpha$ belongs to different subsets of (1.13). For this purpose, in the notation (1.3) we introduce the polynomials

$$
\begin{array}{ll}
q_{0}(z)=\beta_{5}-\beta_{3} z+\beta_{6} z^{2}, & \\
q_{1}(z)=\beta_{2}-\beta_{5} z+\beta_{4} z^{2}, & q_{3}(z)=z q_{2}(z) \\
q_{2}(z)=\beta_{4}-\beta_{6} z+\beta_{1} z^{2}, & q_{4}(z)=q_{0}(z)-z q_{2}(z) .
\end{array}
$$


We can directly check that

$$
-\left(a_{21}+a_{22} z\right)\left(\begin{array}{cc}
p_{2}(z) & -p_{3}(z) \\
-p_{3}(z) & p_{1}(z)
\end{array}\right)=\left(\begin{array}{cc}
-q_{3}(z) & -q_{1}(z) \\
q_{2}(z) & q_{4}(z)
\end{array}\right) .
$$

Lemma 2.2. In the case $(i)$ of distinct roots we can set

$$
\begin{gathered}
b=\left(\begin{array}{cc}
p_{2}\left(\nu_{1}\right) & p_{2}\left(\nu_{2}\right) \\
-p_{3}\left(\nu_{1}\right) & -p_{3}\left(\nu_{2}\right)
\end{array}\right), c=\left(\begin{array}{cc}
-q_{3}\left(\nu_{1}\right) & -q_{3}\left(\nu_{2}\right) \\
q_{2}\left(\nu_{1}\right) & q_{2}\left(\nu_{2}\right)
\end{array}\right), \alpha \in \mathcal{A}_{1}, \\
b=\left(\begin{array}{cc}
-p_{3}\left(\nu_{1}\right) & -p_{3}\left(\nu_{2}\right) \\
p_{1}\left(\nu_{1}\right) & p_{1}\left(\nu_{2}\right)
\end{array}\right), c=\left(\begin{array}{cc}
-q_{1}\left(\nu_{1}\right) & -q_{1}\left(\nu_{2}\right) \\
q_{4}\left(\nu_{1}\right) & q_{4}\left(\nu_{2}\right)
\end{array}\right), \alpha \in \mathcal{A}_{2}, \\
b=1, \quad c=\left(\begin{array}{cc}
-i \sqrt{\alpha_{1} \alpha_{3}} & -\alpha_{3} \\
\alpha_{3} & -i \sqrt{\alpha_{2} \alpha_{3}}
\end{array}\right), \alpha \in \mathcal{A}_{0} .
\end{gathered}
$$

In the case (ii) of a multiple root we can set

$$
b=\left(\begin{array}{cc}
p_{2}(\nu) & p_{2}^{\prime}(\nu) \\
-p_{3}(\nu) & -p_{3}^{\prime}(\nu)
\end{array}\right), c=\left(\begin{array}{cc}
-q_{3}(\nu) & -q_{3}^{\prime}(\nu) \\
q_{2}(\nu) & q_{2}^{\prime}(\nu)
\end{array}\right) .
$$

In all cases the matrix $c$ is invertible and representable in the form

$$
c=c_{0} d,
$$

where

$$
\text { (i) } \quad c_{0}=\left(\begin{array}{cc}
-\nu_{1} & -\nu_{2} \\
1 & 1
\end{array}\right), \quad \text { (ii) } \quad c_{0}=\left(\begin{array}{cc}
-\nu & -1 \\
1 & 0
\end{array}\right)
$$

and the matrix d commutes with $J$.

Proof. Expressions (2.22) and (2.24) for the matrix $b$ follow directly from Lemma 1.2. The fact that for $\alpha \in \mathcal{A}_{0}$ the matrix $b=1$ satisfies (2.3) can be checked directly due to (1.15).

In case $(i)$ for the columns of the matrix $c$ in (2.17) we have the expressions $c_{(j)}=-\left(a_{21}+a_{22} \nu_{j}\right) b_{(j)}$. Substituting here the columns of the matrix $b$ from (2.22) and using relation (2.20), we obtain the respective expressions for the matrices $c$. Equality (2.23) for $c$ can be checked directly.

Similarly, in case $(i i)$ the relations

$$
c_{(1)}=-\left(a_{21}+a_{22} \nu\right) b_{(1)}, \quad c_{(2)}=-\left(a_{21}+a_{22} \nu\right) b_{(2)}-a_{22} b_{(1)},
$$

together with (2.20) and with the expression (2.24) for $b$ yield the desired result.

It remains to establish the second part of the Lemma. By (1.15) for the matrix $c$ in (2.23) we can write

$$
c=\left(\begin{array}{cc}
-\nu_{1} & -\nu_{2} \\
1 & 1
\end{array}\right)\left(\begin{array}{cc}
\alpha_{3} & 0 \\
0 & -i \sqrt{\alpha_{2} \alpha_{3}}
\end{array}\right)
$$

therefore it suffices to consider the cases $\alpha \in \mathcal{A}_{j}, j=1,2$.

In notation (2.20) polynomial (1.8) can be written in the form

$$
\chi(z)=q_{1}(z)-z q_{0}(z)+z^{2} q_{2}(z)=q_{1}(z)-z q_{4}(z) .
$$

In particular, $q_{1}(\nu)=\nu q_{4}(\nu)$ for $\chi(\nu)=0$. Therefore, in the case of distinct roots $\nu_{j}$ matrix $c$ in $(2.22)$ can be written in the form, respectively,

$$
c=\left(\begin{array}{cc}
-\nu_{1} & -\nu_{2} \\
1 & 1
\end{array}\right)\left(\begin{array}{cc}
-p_{2}\left(\nu_{1}\right) & 0 \\
0 & -p_{2}\left(\nu_{2}\right)
\end{array}\right), \quad \alpha \in \mathcal{A}_{1}
$$

and

$$
c=\left(\begin{array}{cc}
-\nu_{1} & -\nu_{2} \\
1 & 1
\end{array}\right)\left(\begin{array}{cc}
p_{4}\left(\nu_{1}\right) & 0 \\
0 & p_{4}\left(\nu_{2}\right)
\end{array}\right), \quad \alpha \in \mathcal{A}_{2} .
$$

If the root $\nu$ is multiple, then $\alpha \in \mathcal{A}_{1} \cap \mathcal{A}_{2}$ and the matrix $c$ in (2.24) can be represented in the form

$$
c=\left(\begin{array}{cc}
-\nu & -1 \\
1 & 0
\end{array}\right)\left(\begin{array}{cc}
p_{3}(\nu) & p_{3}^{\prime}(\nu) \\
0 & p_{3}(\nu)
\end{array}\right) .
$$


Therefore it suffices to check that

$$
\begin{aligned}
& \chi(\nu)=q_{2}(\nu)=0 \Rightarrow \alpha \notin \mathcal{A}_{1}, \\
& \chi(\nu)=q_{4}(\nu)=0 \Rightarrow \alpha \notin \mathcal{A}_{2} .
\end{aligned}
$$

The first three equalities in (2.20) with respect to the vectors $q=\left(q_{2}, q_{1}, q_{0}\right)$ and $e=\left(z^{2}, 1,-z\right)$ can be written in the form $q=\beta e$. In particular, the vector $q(z) \neq 0$ for any $z$. Since $\beta=(\operatorname{det} \alpha) \alpha^{-1}$, one has $(\operatorname{det} \alpha) e=\alpha q$, or, in the explicit form,

$$
\begin{gathered}
(\operatorname{det} \alpha) z^{2}=\alpha_{1} q_{2}+\alpha_{4} q_{1}+\alpha_{6} q_{0}, \\
(\operatorname{det} \alpha)=\alpha_{4} q_{2}+\alpha_{2} q_{1}+\alpha_{5} q_{0}, \\
-(\operatorname{det} \alpha) z=\alpha_{6} q_{2}+\alpha_{5} q_{1}+\alpha_{3} q_{0} .
\end{gathered}
$$

In particular, the polynomials $q_{j}$ are linked by two relations

$$
\begin{aligned}
& \left(\alpha_{4} z+\alpha_{6}\right) q_{2}+\left(\alpha_{2} z+\alpha_{5}\right) q_{1}+\left(\alpha_{5} z+\alpha_{3}\right) q_{2}=0 \\
& \left(\alpha_{6} z+\alpha_{1}\right) q_{2}+\left(\alpha_{5} z+\alpha_{4}\right) q_{1}+\left(\alpha_{3} z+\alpha_{6}\right) q_{2}=0 .
\end{aligned}
$$

Now suppose that $\chi(\nu)=q_{2}(\nu)=0$. Then by $(2.26)$ we must have $q_{1}(\nu)=\nu q_{0}(\nu), q_{0}(\nu) \neq 0$. Therefore due to (1.6) for $z=\nu$ the left-hand side of the first equality (2.28) coincides with $p_{2}(\nu) q_{0}(\nu)$. Thus $p_{2}(\nu)=0$, and from (1.11) follows (2.27).

The argument for the second case is similar. If $\chi(\nu)=q_{4}(\nu)=0$, then by (2.26) one must have $q_{1}(\nu)=0$ and $q_{0}(\nu)=\nu q_{2}(\nu), q_{2}(\nu) \neq 0$. Therefore from (1.6) and from the second equality (2.28), it follows that $p_{1}(\nu)=0$, which means the linear dependence of the polynomials $p_{1}$ and $p_{3}$.

By Theorem 2.2, the space of $J$-analytic functions $\phi$ such that the real function $v=\operatorname{Re} c \phi$ is the identical zero is three-dimensional and consists of polynomials

$$
\phi(z)=\eta_{0}+z_{J} \eta_{1}, \quad \operatorname{Re} c \eta_{0}=0, \operatorname{Re} c \eta_{1}=\operatorname{Re} c J \eta_{1}=0 .
$$

This fact can be specified to some extent.

Lemma 2.3. The space $\left\{\eta \in \mathbb{C}^{2} \mid \operatorname{Re} c \eta=\operatorname{Re} c J \eta=0\right\}$ is the one-dimensional hull of the vector $e$ defined by the system

$$
c e=i \xi^{0}, c J e=i \xi^{1},
$$

where in the notation of $(1.7)$ the vectors $\xi^{k} \in \mathbb{R}^{2}(k=0,1)$ are defined by equalities

$$
\xi^{0}=(-\operatorname{Im} t, \operatorname{Im} s), \quad \xi^{1}=(-\operatorname{Im}(\bar{s} t), \operatorname{Im} t) .
$$

In particular, in formula (2.29) one can set $\eta_{1}=\lambda e, \lambda \in \mathbb{R}$.

Simultaneous equalities $\operatorname{Re} c \eta=\operatorname{Re} c J \eta=\operatorname{Re} c J^{2} \eta=0$ are possible only for $\eta=0$.

Proof. By (2.25), the operation $\eta \rightarrow d \eta$ takes the subspace $X_{n} \subseteq \mathbb{C}^{2}$ defined by the conditions $\operatorname{Re} c J^{k} \eta=0,0 \leq k \leq n$, to a similar space corresponding to $c_{0}$. In the case $c=c_{0}$ equalities $\operatorname{dim} X_{1}=1$ and $\operatorname{dim} X_{2}=0$ can be checked directly.

Further we note that the system of equations (2.30) is solvable if and only if one has

$$
\left(c J c^{-1}\right) \xi^{0}=\xi^{1} .
$$

But by (2.25) in both cases $(i)$ and (ii) the matrix

$$
c J c^{-1}=c_{0} J c_{0}^{-1}=\left(\begin{array}{cc}
s & t \\
-1 & 0
\end{array}\right),
$$

and equality (2.31) for the vectors $\xi^{k}$ under consideration can be checked directly. 
Consider system (2.1), (2.2) in more detail. If the roots $\nu_{j}$ are distinct, then this system reduces into two scalar equations

$$
\frac{\partial \phi}{\partial y}-\nu_{j} \frac{\partial \phi}{\partial x}=0
$$

which pass into the Cauchy-Riemann equation in the variables $\tilde{x}+i \tilde{y}=x+\nu_{j} y$. Therefore the substitution

$$
\phi_{j}(x, y)=\psi_{j}\left(x+\nu_{j} y\right), \quad j=1,2,
$$

defines analytic functions $\psi_{j}$ in the domains $D\left(\nu_{j}\right)=\left\{\tilde{z}=x+\nu_{j} y, z \in D\right\}$.

A direct check shows that in the case of a multiple root $\nu$ the substitution

$$
\phi_{1}(x, y)=\psi_{1}(x+\nu y)+y \psi_{2}^{\prime}(x+\nu y), \phi_{2}(x, y)=\psi_{2}(x+\nu y)
$$

is one-to-one between the $J$-analytic vector function $\phi$ and the vector function $\psi$, which is analytic in the domain $D(\nu)$. The inverse transform is given by a similar formula

$$
\psi_{1}(x+\nu y)=\phi_{1}(x, y)-y \phi_{2}^{\prime}(x, y), \psi_{2}(x+\nu y)=\phi_{2}(x, y) .
$$

It is worth noting that in the case where the domain $D$ is the upper half-plane the function $\psi$ is also defined in the same half-plane.

Substitution of formulas (2.32), (2.33) into (2.10), (2.17) together with (2.13) leads to classical representations of the shift vector and the stress tensor by means of a pair of analytic functions. In the case of multiple roots the derivative of one of the functions also enters this representation. It is well known $[11,12]$ that this circumstance somewhat interferes with the use of these representations in the study of boundary value problems.

As an illustration we discuss the connection between representations (2.12), (2.15) for an isotropic medium and the classical Kolosov-Muskhelishvili formulas expressing the shift vector $u$ and the stress tensor $\sigma$ by analytic functions. According to Section 1 in this case we have a multiple root $\nu=i$ and the matrix $J$ is a Jordan block, while the matrices $b$ and $c$ are given by formulas $(2.24)$.

By (1.20), (1.26) these formulas yield the equalities

$$
b=\left(\begin{array}{cc}
\alpha_{3}-\alpha_{1} & 2 \alpha_{1} i \\
\left(\alpha_{3}-\alpha_{1}\right) i & \alpha_{3}-\alpha_{1}
\end{array}\right), \quad c=2 \alpha_{3}\left(\begin{array}{cc}
\left(\alpha_{1}-\alpha_{3}\right) i & 2 \alpha_{1}-\alpha_{3} \\
\alpha_{3}-\alpha_{1} & \alpha_{1} i
\end{array}\right) .
$$

By Lemma 2.1, one can take as $b$ and $c$ the matrices obtained from the multiplication of these equalities from the right by the matrix

$$
d=\left(\alpha_{3}-\alpha_{1}\right)^{-1}\left(\begin{array}{cc}
1 & 2 \alpha_{1}\left(\alpha_{1}-\alpha_{3}\right)^{-1} i \\
0 & 1
\end{array}\right) .
$$

After elementary calculations we finally arrive at the formulas

$$
b=\left(\begin{array}{cc}
1 & 0 \\
i & -\varkappa
\end{array}\right), \quad c=\alpha_{3}\left(\begin{array}{cc}
-2 i & \varkappa-1 \\
2 & i(\varkappa+1)
\end{array}\right)
$$

with a positive constant $\varkappa$ from $(1.27)$.

With these matrices written component-wise representation (2.10) takes the form

$$
u_{1}=\operatorname{Re} \phi_{1}, \quad u_{2}=\operatorname{Re}\left(i \phi_{1}-\varkappa \phi_{2}\right) .
$$

Substituting the expression (2.34) of the matrix $c$ into (2.17), for the elements of the matrix $\sigma$ in $(2.16)$ we obtain the representations

$$
\begin{gathered}
\sigma_{1}=\operatorname{Re}\left[2 \phi_{1}^{\prime}+i(\varkappa-3) \phi_{2}^{\prime}\right], \\
\sigma_{2}=-\operatorname{Re}\left[2 \phi_{1}^{\prime}+i(\varkappa+1) \phi_{2}^{\prime}\right], \\
\sigma_{3}=\operatorname{Re}\left[2 i \phi_{1}^{\prime}-(\varkappa-1) \phi_{2}^{\prime}\right)
\end{gathered}
$$

of the components of the stress tensor. It is worth noting that the matrix $c$ possesses the property $(c J)_{2 k}=-c_{1 k}$, and therefore the equality which defines $\sigma_{3}$ enters the component-wise notation $(2.35)$ twice. 
Substituting (2.33) into (2.34), (2.36), we arrive at the representation

$$
u_{1}=\operatorname{Re}\left[\psi_{1}+y \psi_{2}^{\prime}\right], u_{2}=\operatorname{Re}\left[i\left(\psi_{1}+y \psi_{2}^{\prime}\right)-\varkappa \psi_{2}\right]
$$

of the components of the shift vector and

$$
\begin{gathered}
\sigma_{1}=\alpha_{3} \operatorname{Re}\left[2\left(\psi_{1}^{\prime}+y \psi_{2}^{\prime \prime}\right)+i(\varkappa-3) \psi_{2}^{\prime}\right], \\
\sigma_{2}=-\alpha_{3} \operatorname{Re}\left[2\left(\psi_{1}^{\prime}+y \psi_{2}^{\prime \prime}\right)+i(\varkappa+1) \psi_{2}^{\prime}\right], \\
\sigma_{3}=\alpha_{3} \operatorname{Re}\left[2 i\left(\psi_{1}^{\prime}+y \psi_{2}^{\prime \prime}\right)-(\varkappa-1) \psi_{2}^{\prime}\right]
\end{gathered}
$$

of the elements of the stress tensor by a pair of analytic functions $\psi_{1}, \psi_{2}$ of the same variable $z$.

With the help of a linear substitution

$$
\chi_{1}(z)=-i \psi_{2}(z), \chi_{2}(z)=-2 \psi_{1}(z)+i \varkappa \psi_{2}(z)+i z \psi_{2}^{\prime}(z)
$$

these representations can be rewritten in the form of the equalities

$$
\begin{gathered}
2\left(u_{1}-i u_{2}\right)(z)=\varkappa \overline{\chi_{1}(z)}-z \chi_{1}^{\prime}(z)-\chi_{2}(z), \\
\left(\sigma_{1}+\sigma_{2}\right)(z)=4 \alpha_{3} \operatorname{Re} \chi_{1}^{\prime}(z), \quad\left(\sigma_{2}-\sigma_{1}+2 i \sigma_{3}\right)(z)=2 \alpha_{3}\left[\bar{z} \chi_{1}^{\prime \prime}(z)+\chi_{1}^{\prime}(z)\right],
\end{gathered}
$$

i.e., of the classical Kolosov-Muskhelishvili formulas [12].

\section{Analytic Functions According to Douglis}

Let a domain $D$ be bounded by a smooth contour $\Gamma$, which is positively oriented with respect to $D$. This domain can be either finite (i.e., lies inside some disk) or infinite (i.e., contains the exterior of some disk and therefore be a neighborhood of the infinite point $\infty$ on the plane). It is convenient to denote these two possible cases for brevity $\sigma(D)=1$ and $\sigma(D)=0$, respectively.

Consider in the domain $D$ system (2.2) with an arbitrary matrix $J \in \mathbb{C}^{l \times l}$ such that all its eigenvalues lie in the upper half-plane. In the case where $J$ is a Hankel matrix, this system was studied by Douglis [4] in the framework of hypercomplex numbers. It is convenient to relate the complex number $z=x+i y$ to the matrix

$$
z_{J}=x 1+y J
$$

where $x=x 1$ is a scalar matrix. Since the eigenvalues of $J$ lie in the upper half-plane, for $z \neq 0$ this matrix is invertible. Recall that the solutions $\phi$ of system $(2.2)$ were called $J$-analytic functions. The introduction of this term is motivated by the fact that these solutions can be described as functions of the class $C^{1}(D)$ that admit at each point $z \in D$ the generalized derivative

$$
\phi^{\prime}(z)=\lim _{t \rightarrow z}(t-z)_{J}^{-1}[\phi(t)-\phi(z)]
$$

which coincides with the partial derivative in $x$. In the case $\sigma(D)=0$ of an unbounded domain we add to this definition the condition that $\phi$ should be bounded at $\infty$. In the sequel we will see that in this case $\phi$ has a limit $\phi(\infty)=\lim \phi(z)$ as $z \rightarrow \infty$. In general, we will say that $\phi$ has the order $k \in \mathbb{Z}$ at $\infty$ if the function $|z|^{-k} \phi(z)$ is bounded in a neighborhood of $\infty$.

Let the $l$-vector function $\phi \in C^{1}(D)$ satisfy system $(2.2)$ in the domain $D$ and in the case $\sigma(D)=0$ let it have the order -2 at infinity. Then, integrating equality (2.2) and using Green's formula, we will obtain the equality

$$
\int_{\Gamma} d t_{J} \phi^{+}(t)=0
$$

which plays the role of the Cauchy Theorem. Here the matrix differential is defined similarly to (3.1) and acts on the vector $\phi^{+}$by the usual rule and therefore stands before the vector. As in the case of classical analytic functions, one can deduce the Cauchy Formula

$$
\frac{1}{2 \pi i} \int_{\Gamma}(t-z)_{J}^{-1} d t_{J} \phi^{+}(t)=\left\{\begin{array}{cl}
\phi(z), & z \in D \\
0, & z \in \widetilde{D}
\end{array}\right.
$$


where $\widetilde{D}=\mathbb{C} \backslash \bar{D}$ and it is assumed in addition that in the case $\sigma(D)=0$ the function $\phi$ has the order -1 at infinity. This formula, in particular, implies that $\phi \in C^{\infty}(D)$. Denoting by $\phi^{(k)}$ the consecutive partial derivatives in $x$, due to (2.2) we have expressions

$$
\frac{\partial^{k} \phi}{\partial x^{k-s} \partial y^{s}}=J^{s} \phi^{(k)}, \quad 0 \leq s \leq k .
$$

for the other partial derivatives. The Cauchy Formula also implies that a $J$-analytic function on the whole plane that vanishes at infinity is the identical zero. As for usual analytic functions, formulas (3.2), (3.3) easily imply the following proposition on analytic continuation.

Let the domain $D$ contain a cut $L$, i.e., a smooth arc ending in the points of the boundary contour $\Gamma$, which lies inside $D$ with the exception of the endpoints themselves. Then, if the function $\phi$ is continuous in $D$ and $J$-analytic in $D \backslash L$, then this function is $J$-analytic in the whole domain $D$.

All the main results of the classical theory of analytic functions based on the Cauchy integral remain valid for $J$-analytic functions [17]. For convenience, we formulate the main ideas of this theory without proof.

In a neighborhood of an isolated singular point $a$ for a $J$-analytic function we have a Laurent decomposition

$$
\phi(z)=\sum(z-a)_{J}^{k} c_{k}, \quad c_{k} \in \mathbb{C}^{l},
$$

in integer powers of the matrix $(z-a)_{J}$. If $\phi$ is bounded in the neighborhood of this point, then the singularity is removable and this decomposition becomes the respective Taylor series with coefficients $c_{k}=\phi^{(k)} / k$ !. The respective partial sums of this series are $J$-analytic polynomials

$$
p(z)=\sum_{k=0}^{n}(z-a)_{J}^{k} c_{k}, \quad c_{k} \in \mathbb{C}^{l} .
$$

If the domain $D$ is unbounded, the infinite point $\infty$ can be considered as an isolated one. In this case, the Laurent decomposition holds in a neighborhood of $\infty$ in integer powers of $z_{J}$. If $\phi$ has the order $k$ at infinity, then this decomposition contains only powers $z_{J}^{i}, i \leq k$. In particular, the function $z_{J}^{-k} \phi(z)$ is Douglas analytic in a neighborhood of $\infty$.

If the function $\psi$ is given and $J$-analytic in a simply connected domain $D$, then the integral

$$
\phi(z)=\int_{z_{0}}^{z} d t_{J} \psi(t)
$$

does not depend on the integration path and defines a $J$-analytic function with a derivative $\phi^{\prime}=\psi$. In the case of a multiply connected domain $D$ the primitive $\phi$ of the function $\psi$, generally speaking, is multivalued and admits bifurcations while encircling connected components of the boundary of the domain. Evidently, formula (3.5) yields a single-valued function if and only if one has

$$
\int_{\Gamma^{\prime}} d t_{J} \psi(t)=0
$$

for any simple contour $\Gamma^{\prime} \subseteq D$. In the general case the integral here can be understood as the increment of the function $\phi$ along the contour $\Gamma^{\prime}$.

Let the domain $D$ be bounded and let its boundary consist of the finite number $m$ of connected components. Consider in $D$ simple contours $\Gamma_{j}^{\prime}, 1 \leq j \leq m-1$, that contain within themselves the respective $m-1$ components among these. Then by the Cauchy Theorem it suffices to check condition (3.6) for these contours. A similar statement holds for an unbounded domain provided that $\psi$ has the order -2 at infinity. In this case one can integrate in (3.5) starting at $z_{0}=\infty$, and the components of the boundary linked to $\Gamma_{j}^{\prime}$ are equivalent. 
Similarly to (3.3), one can introduce a generalized Cauchy type integral

$$
(I \varphi)(z)=\frac{1}{2 \pi i} \int_{\Gamma}(t-z)_{J}^{-1} d t_{J} \varphi(t)
$$

defining outside the oriented contour a $J$-analytic function $\phi=I \varphi$ with the order -1 at infinity and the respective singular Cauchy integral

$$
(S \varphi)\left(t_{0}\right)=\frac{1}{\pi i} \int_{\Gamma}\left(t-t_{0}\right)_{J}^{-1} d t_{J} \varphi(t), \quad t_{0} \in \Gamma,
$$

understood in the sense of the principal value. The operator $I$ defined by this integral is bounded in the Hölder spaces $C^{\mu}(\Gamma) \rightarrow C^{\mu}(\bar{D}), 0<\mu<1$, where $D$ is any connected component of the complement of $\Gamma$, and for its boundary values $\phi^{ \pm}$(the signs are defined by the orientation of the contour) the Sohocki-Plemelj formula holds (see [1]):

$$
2 \phi^{ \pm}= \pm \varphi+S \varphi
$$

Let a function $\phi$ be Douglis analytic outside of the contour $\Gamma$, have a finite order at infinity and belong to $C^{\mu}(\bar{D})$, where $D$ is any connected component of the complement to $\Gamma$. Then by $(3.7)$ the difference $\phi_{0}=\phi-I \varphi$, where $\varphi=\phi^{+}-\phi^{-}$, has the property $\phi_{0}^{+}=\phi_{0}^{-}$. By the Cauchy formulas (3.2), (3.3), applied to domains $D$, it is easy to deduce from here that $\phi_{0}$ is Douglis analytic in the whole plane and therefore is a $J$-analytic polynomial $p$.

Formula (3.7) also implies that the singular operator $S$ is bounded in $C^{\mu}(\Gamma)$. By the way, with the help of the next lemma this fact can be easily reduced to a similar well-known result [13] for the classical Cauchy singular operator

$$
\left(S_{0} \varphi\right)\left(t_{0}\right)=\frac{1}{\pi i} \int_{\Gamma} \frac{\varphi(t) d t}{t-t_{0}}, \quad t_{0} \in \Gamma,
$$

which corresponds to the case $J=i$.

Lemma 3.1. Let the contour $\Gamma$ belong to the class $C^{1, \nu}$ and

$$
k\left(t_{0}, t\right) \in C^{\nu}(\Gamma \times \Gamma), \quad k(t, t) \equiv 0 .
$$

Then the integral operator

$$
(K \varphi)\left(t_{0}\right)=\frac{1}{\pi i} \int_{\Gamma} \frac{k\left(t_{0}, t\right)}{t-t_{0}} \varphi(t) d t, \quad t_{0} \in \Gamma,
$$

is bounded $C(\Gamma) \rightarrow C^{\nu}(\Gamma)$ and, in particular, compact in the spaces $C(\Gamma)$ and $C^{\mu}(\Gamma), 0<\mu<\nu$.

If the function $\varphi$ is summable, then integral (3.9) exists almost everywhere in $\Gamma$ and the operator $K$ is compact in the Lebesgue space $L^{p}(\Gamma)$ for any $p \geq 1$.

Proof. There exists a $0<\rho<1 / 2$ (the standard radius of the contour) such that for any point $a \in \Gamma$ and $0<\delta<\rho$ the set $\Gamma \cap\{|t-a| \leq \delta\}$ is a smooth arc. Then for the function $\psi=K \varphi$ we have an obvious estimate

$$
\left|\psi\left(t_{0}\right)\right| \leq|k|_{\nu}|\varphi|_{0} \int_{\Gamma}\left|t-t_{0}\right|^{\nu-1}|d t| \leq M_{0}|k|_{\nu}|\varphi|_{0},
$$

where ||$_{0}$ and ||$_{\nu}$ denote the norms in $C$ and $C^{\nu}$, respectively, and the constant $M_{0}>0$ depends only on $\Gamma$. Further we use the estimate

$$
\int_{\Gamma \cap\{|t-a| \geq \delta\}}|t-a|^{\alpha-2}|d t| \leq M_{1}\left\{\begin{array}{cc}
\delta^{\alpha-1}, & 0<\alpha<1, \\
\ln (1 / \delta), & \alpha=1,
\end{array}\right.
$$

where the constant $M_{1}>0$ depends only on $\Gamma$ and on $\alpha$. 
Fix the points $t_{1}, t_{2} \in \Gamma$, and let $\delta=\left|t_{1}-t_{2}\right| \leq \rho / 3$. Write

$$
\psi\left(t_{1}\right)-\psi\left(t_{2}\right)=\int_{\Gamma}\left[\frac{k\left(t_{1}, t\right)}{t-t_{1}}-\frac{k\left(t_{2}, t\right)}{t-t_{2}}\right] \varphi(t) d t=\Delta_{1}+\Delta_{2},
$$

where $\Delta_{1}$ and $\Delta_{2}$ denote integrals along $\Gamma_{1}=\Gamma \cap\left\{\left|t-t_{1}\right| \leq 2 \delta\right\}$ and $\Gamma_{2}=\Gamma \cap\left\{\left|t-t_{1}\right| \geq 2 \delta\right\}$, respectively. Evidently,

$$
\left|\Delta_{1}\right| \leq|k|_{\nu}|\varphi|_{0} \int_{\Gamma_{1}}\left[\left|t-t_{1}\right|^{\nu-1}+\left|t-t_{2}\right|^{\nu-1}\right]|d t| .
$$

Since $\left|t-t_{1}\right| \leq 2 \delta$ implies $\left|t-t_{2}\right| \leq 3 \delta$, similarly to (3.10) we have:

$$
\left|\Delta_{1}\right| \leq M_{0}\left[(2 \delta)^{\nu}+(3 \delta)^{\nu}\right]|k|_{\nu}|\varphi|_{0}
$$

As for $\Delta_{2}$, we write

$$
\Delta_{2}=\int_{\Gamma_{2}} \frac{k\left(t_{1}, t\right)-k\left(t_{2}, t\right)}{t-t_{1}} \varphi(t) d t+\int_{\Gamma_{2}} \frac{\left(t_{1}-t_{2}\right) k\left(t_{2}, t\right)}{\left(t-t_{1}\right)\left(t-t_{2}\right)} \varphi(t) d t .
$$

Then we have

$$
\left|\Delta_{2}\right| \leq|k|_{\nu}|\varphi|_{0}\left[\delta^{\nu} \int_{\Gamma_{2}}\left|t-t_{1}\right|^{-1}|d t|+\delta \int_{\Gamma_{2}}\left|t-t_{1}\right|^{-1}\left|t-t_{2}\right|^{\nu-1}|d t|\right] .
$$

Since $\left|t-t_{1}\right| \geq 2 \delta$ implies $\left|t-t_{2}\right| \geq\left|t-t_{1}\right|-\delta \geq\left|t-t_{1}\right|-\left|t-t_{1}\right| / 2$, we can apply estimate (3.11) to the expression in square brackets. Then we have

$$
\left|\Delta_{2}\right| \leq|k|_{\nu}|\varphi|_{0} M_{1}\left[\delta^{\nu} \ln (1 / \delta)+2^{1-\nu} \delta^{\nu}\right] .
$$

Combining both estimates for $\Delta_{1}$ and $\Delta_{2}$ results in

$$
\left|\psi\left(t_{1}\right)-\psi\left(t_{2}\right)\right| \leq C|k|_{\nu}|\varphi|_{0}\left|t_{1}-t_{2}\right|^{\mu} \quad \text { if } \quad\left|t_{1}-t_{2}\right| \leq \rho / 3
$$

with some constant $C>0$. By (3.10), this implies boundedness of the operator $K$ from $C$ into $C^{\nu}$.

We turn to the second statement of the lemma. Assuming that the function $\varphi$ is summable, according to (3.8) we write (3.9) in the form

$$
(K \varphi)\left(t_{0}\right)=\int_{\Gamma} \frac{\tilde{k}\left(t_{0}, t\right) \varphi(t)|d t|}{\left|t-t_{0}\right|^{\alpha}}
$$

where $1-\nu<\alpha<1$, and the function $\tilde{k}$ is continuous and vanishes at $t=t_{0}$. Then by the Fubini Theorem this integral exists almost everywhere and defines a summable function $\psi=K \varphi$ with an estimate

$$
\int_{\Gamma}\left|\psi\left(t_{0}\right)\right|\left|d t_{0}\right| \leq|\tilde{k}|_{0} \int_{\Gamma}|\varphi(t)||d t| \int_{\Gamma}\left|t-t_{0}\right|^{-\alpha}\left|d t_{0}\right| \leq M|\tilde{k}|_{0} \int_{\Gamma}|\varphi(t)||d t| .
$$

If $\varphi \in L^{p}(\Gamma), p>1$, then by the Hölder inequality

$$
\left|\psi\left(t_{0}\right)\right| \leq|\tilde{k}|_{0}\left(\int_{\Gamma} \frac{|\varphi(t)|^{p}|d t|}{\left|t-t_{0}\right|^{\alpha}}\right)^{1 / p}\left(\int_{\Gamma} \frac{|d t|}{\left|t-t_{0}\right|^{\alpha}}\right)^{1 / q},
$$

where $1 / q=1-1 / p$. Rasing this inequality to the $p$-th power and integrating, due to the previous inequality we get

$$
\int_{\Gamma}\left|\psi\left(t_{0}\right)\right|^{p}\left|d t_{0}\right| \leq|\tilde{k}|_{0}^{p} M^{p / q} \int_{\Gamma}|\varphi(t)|^{p}|d t| .
$$


Thus the operator $K$ is bounded in $L^{p}(\Gamma)$ with an estimate

$$
|K|_{\mathcal{L}\left(L^{p}\right)} \leq M|\tilde{k}|_{0}
$$

for its norm.

Evidently, if for some $\delta>0$ the function $\tilde{k}\left(t_{0}, t\right)$ vanishes for $\left|t-t_{0}\right| \leq \delta$, then the operator $K$ is compact in $L^{p}(\Gamma)$. We choose a sequence of functions $\tilde{k}_{n}$ with this property with respect to $\delta=\delta_{n}$, which converges to $\tilde{k}$ in the sup-norm, and let $K_{n}$ be defined from $\tilde{k}_{n}$, as above. Then by estimate (3.12) applied to the difference $K-K_{n}$ the sequence of compact operators $K_{n}$ converges to $K$ in the operator norm, so that the operator $K$ is compact.

Lemma 3.2. Let the contour $\Gamma$ belong to the class $C^{1, \nu}$ and let the eigenvalues of the matrix $J$ lie not on the real axis. Then the operator $S-S_{0}$ is compact in the spaces $C^{\mu}(\Gamma), \quad 0<\mu<\nu$, and $L^{p}(\Gamma)$, $p>1$.

If the contour $\Gamma$ is the boundary of the domain $D$ and positively oriented with respect to it, then $S^{2}=S_{0}^{2}=1$.

Proof. We write the difference $S-S_{0}$ in the form

$$
\left.\left[\left(S-S_{0}\right) \varphi\right)\right]\left(t_{0}\right)=\frac{1}{\pi i} \int_{\Gamma} \frac{q\left(t ; t-t_{0}\right)}{t-t_{0}} \varphi(t) d t
$$

with a matrix function $q(t, \xi)=[e(t)]_{J} \xi_{J}^{-1} \xi-e(t)$. Then it remains to make use of Lemma 3.1 and the following general property of functions of the form $q$.

Let $\Gamma \in C^{1, \nu}$ and the function $q\left(t_{0}, t ; \xi\right)$ defined for $t_{0}, t \in \Gamma$ and $\xi=\xi_{1}+i \xi_{2} \in \mathbb{C}, \xi \neq 0$ be even, homogeneous of order zero, and continuously differentiable in $\xi$, namely, continuous in all the variables together with its partial derivatives in $\xi$. Then, if $q\left(t_{0}, t ; \xi\right) \in C^{\nu}(\Gamma \times \Gamma)$ uniformly in $|\xi|=1$, then the function $k\left(t_{0}, t\right)=q\left(t_{0}, t ; t-t_{0}\right)$ belongs to $C^{\nu}(\Gamma \times \Gamma)$, and $k(t, t)=q(t, t ; e(t)$, where $e(t)$ is the unit tangential vector on $\Gamma$.

It suffices to prove this proposition in a neighborhood of a fixed point $(a, a) \in \Gamma \times \Gamma$. Write a parametrization of the contour in this neighborhood in the form $z=\gamma(s),|s| \leq \delta$, where $s$ is the parameter of the arc length measured from the point $a$. Then the function

$$
\alpha\left(s_{0}, s\right)=\frac{\gamma(s)-\gamma\left(s_{0}\right)}{s-s_{0}}=\int_{0}^{1} \gamma^{\prime}\left[s \tau+s_{0}(1-\tau)\right] d \tau
$$

belongs to the class $C^{\nu}$ in the square $\left|s_{0}\right|,|s| \leq \delta$, is separated from zero in absolute value and takes the value $\gamma^{\prime}(s)$ at $s_{0}=s$. Consequently, the function $k\left[\gamma\left(s_{0}\right), \gamma(s)\right]=q\left[\gamma\left(s_{0}\right), \gamma(s) ; \alpha\left(s_{0}, s\right)\right]$ also belongs to this class and its value at $s_{0}=s$ coincides with $q\left[\gamma\left(s_{0}\right), \gamma(s) ; \gamma^{\prime}(s)\right]$. Hence it follows that the formulated proposition holds for the function $q\left(t_{0}, t ; t-t_{1}\right)$ in the neighborhood of the contour under consideration.

We turn to the second statement of the Lemma. For the operator $S_{0}$ it is well known [13], and for $S$ it can be proved according to the same scheme as in [13]. Namely, let the contour $\Gamma$ be positively oriented with respect to the domain $D$ and for brevity $P=(1+S) / 2$. Then for the boundary value of the function $\phi=I \varphi$ we have the equality $\phi^{+}=P \varphi$. On the other hand, by the Cauchy formula $\phi=I \phi^{+}$, where we take into account the fact that in the case of an unbounded domain $D$ the function $\phi$ vanishes at $\infty$. Then $P \phi^{+}=\phi^{+}$or $P^{2} \varphi=P \varphi$, which is equivalent to the operator equality $S^{2}=1$.

The boundary properties of integrals of the Cauchy type $I \varphi$ with density from $\varphi \in L^{p}(\Gamma), 1<$ $p<\infty$, were studied in [16]. In this case the $J$-analytic function $\phi=I \varphi$ belongs to the Hardy space $H^{p}(D)$, which can be introduced [18] in the following way. Let the contour $\Gamma$ belong to the class $C^{1, \nu}$ 
and let the sequence of contours $\Gamma_{n} \subseteq D, n=1,2, \ldots$ converge to $\Gamma$ in the metric of $C^{1}$. By definition, this means that for some diffeomorphisms $\gamma_{n}: \Gamma \rightarrow \Gamma_{n}$, the condition

$$
\lim _{n \rightarrow \infty}\left|\gamma_{n}(t)-t\right|_{C^{1}(\Gamma)}=0
$$

holds. Then $H^{p}(D)$ consists of all $J$-analytic functions in $D$ such that the norm

$$
|\phi|=\sup _{n}|\phi|_{L^{p}\left(\Gamma_{n}\right)}
$$

is finite.

In this notation the Cauchy type integral as a linear operator $\varphi \rightarrow \phi=I \varphi$ is bounded $L^{p}(\Gamma) \rightarrow$ $H^{p}(D)$, the angular limiting values $\phi^{ \pm}$exist almost all in $\Gamma$, and the Sohocki-Plemelj formula holds [16]. Conversely, any function $\phi \in H^{p}$ is representable as a Cauchy type integral with density $\varphi \in L^{p}(\Gamma)$.

In fact, let the domain $D_{n} \subseteq D$ be bounded by the contour $\Gamma_{n}$. Then for a fixed point $z \in D$ and sufficiently large $n$ one can write the Cauchy Formula

$$
\phi(z)=\frac{1}{2 \pi i} \int_{\Gamma_{n}}(t-z)_{J}^{-1} d t_{J} \phi(t) .
$$

From (3.14) and the weak compactness [15] of the unit ball in the reflexive Banach space $L^{p}, p>1$, it follows that there exists a function $\varphi \in L^{p}(\Gamma)$ such that some subsequence $\phi \circ \gamma_{n_{k}}$ weakly converges to $\varphi$ in $L^{p}$. Therefore due to (3.13) we can pass to the limit as $k \rightarrow \infty$ in the previous equality for $n=n_{k}$ and as a result represent $\phi$ by a Cauchy type integral.

This implies, in particular, that $\phi \in H^{p}(D)$ if and only if angular limiting values exist almost everywhere in $\Gamma$, belong to $L^{p}(\Gamma)$, and the Cauchy Formula remains valid. By the same reasons, the space $H^{p}$ can be defined as the closure of the class of $J$-analytic functions, which are continuous in the closed domain $\bar{D}$, in the norm

$$
|\phi|=\left|\phi^{+}\right|_{L^{p}(\Gamma)}
$$

equivalent to norm (3.14). Recall that in the case $\sigma(D)=0$ of an unbounded domain $J$-analytic functions are supposed to be bounded at $\infty$, so that the given equality defines a norm in this case as well.

It is well known [13] that any analytic function can be represented by a Cauchy type integral up to an additive constant. This fact also holds [16] for $J$-analytic functions in the Hölder classes. With the help of the following lemma it can be easily extended to the Hardy class as well.

Lemma 3.3. Let a domain $D$ be bounded by a simple Lyapunov contour $\Gamma$ and a matrix $J$ be triangular. Let a J-analytic function $\phi \in H^{p}(D)$ be such that $\operatorname{Re} \phi^{+}$is constant in $\Gamma$. Then $\phi$ is constant in the domain $D$.

Proof. Prove this Lemma first for the scalar case $l=1$, where $J=\nu \in \mathbb{C}$ and $\phi$ is a solution of the equation

$$
\frac{\partial \phi}{\partial y}-\nu \frac{\partial \phi}{\partial x}=0
$$

Under the affine transformation $z=x+i y \rightarrow z_{\nu}=x+\nu y$ this equation becomes the Cauchy-Riemann equation that defines analytic functions. Evidently, the Hardy class is invariant with respect to these transformations, so that without loss of generality the function $\phi$ can be considered analytic. In this case the statement of the Lemma is well known [8].

So in the scalar case the statement of the Lemma is proven. In the general case let for definiteness the matrix $J \in \mathbb{C}^{l \times l}$ be upper triangular, i.e., $J_{i k}=0$ for $i>k$. Then in the coordinate notation with respect to the vector $\phi=\left(\phi_{1}, \ldots, \phi_{l}\right)$ the Douglis system can be rewritten in the form

$$
\frac{\partial \phi_{j}}{\partial y}-\sum_{k=j}^{l} J_{k j} \frac{\partial \phi_{k}}{\partial x}=0, \quad 1 \leq k \leq l .
$$


By the above the last equation of this system implies that the function $\phi_{l}$ is constant. Therefore the $(l-1)$-th equation of this system becomes the scalar equation considered above with respect to $\phi_{l-1}$ and $\nu=J_{l-1, l-1}$. Hence, by the same reasons the function $\phi_{l-1}$ is constant. Repeating the same arguments, we finally come to the conclusion that all the functions $\phi_{k}$ are constant.

We turn to the problem of representability of $J$-analytic functions $\phi \in H^{p}$ by the Cauchy type integrals with real density.

Theorem 3.1. Let a Lyapunov contour $\Gamma$ be the boundary of a domain D, positively oriented with respect to $D$, and consist of components $\Gamma_{1}, \ldots, \Gamma_{m}, m \geq 1$, where in the case of a bounded domain the contour $\Gamma_{m}$ encompasses all the others. Let a matrix $J$ be triangular. Then any $J$-analytic function $\phi \in H^{p}(D)$ can be represented in the form

$$
\phi=I \varphi+\eta, \quad \eta \in \mathbb{C}^{l},
$$

where the real l-vector function $\varphi \in L^{p}(\Gamma)$ and $\operatorname{Re} \eta=0$ in the case of a bounded domain D.

In this representation $\phi=0$ if and only if $\eta=0$ and the function $\varphi$ is constant on the contours $\Gamma_{j}$, and in the case of a bounded domain $D$ it vanishes on $\Gamma_{m}$.

Proof. It follows the same scheme as in the case of functions considered in the Hölder classes [16]. First suppose that the domain $D$ is bounded by a simple contour (i.e., $m=1$ ). Denote by $\widetilde{D}$ the complement of $\bar{D}$ on the plane, and let $\widetilde{I} \varphi$ denote the respective Cauchy type operator in the domain $\widetilde{D}$. Then due to $(3.7)$ one has

$$
(I \varphi)^{+}-(\widetilde{I} \varphi)^{-}=\varphi
$$

We assert that

$$
\begin{gathered}
\operatorname{Re}(I \varphi)^{+}=0 \Rightarrow \varphi=0, \\
\operatorname{Re}(\widetilde{I} \varphi)^{-}=\xi \in \mathbb{R}^{l} \Rightarrow \xi=0, \varphi \in \mathbb{R}^{l} .
\end{gathered}
$$

In fact, if $\operatorname{Re}(I \varphi)^{+}=0$, then by Lemma 3.3 the function $I \varphi$ is constant and due to (3.16) the function $\operatorname{Im}(\widetilde{I} \varphi)^{-}$is also constant. Using Lemma 3.3 again, we derive that the function $\widetilde{I} \varphi$ and consequently the density $\varphi=\xi \in \mathbb{R}^{l}$ are constant as well. But then $I \varphi=\xi$ and since by condition $\operatorname{Re}(I \varphi)^{+}=0$, hence follows (3.17). The argument for the integral $\widetilde{I} \varphi$ is similar. As above, we check that the functions $\widetilde{I} \varphi$ and $\varphi$ are constant. Since the first of them vanishes at infinity, this implies (3.18).

Consider the operators $M \varphi=\operatorname{Re}(I \varphi)^{+}$and $\widetilde{M} \varphi=\operatorname{Re}(\widetilde{I} \varphi)^{-}$that act in $L^{p}(\Gamma)$. According to (3.7), we have:

$$
M \varphi=\operatorname{Re}(\varphi+S \varphi) / 2, \quad \widetilde{M} \varphi=\operatorname{Re}(-\varphi+S \varphi) / 2
$$

The operation of complex conjugation $\varphi \rightarrow \bar{\varphi}$ of the functions induces the respective operator involution $N \rightarrow \bar{N}$ by the rule $\bar{N} \varphi=\bar{N} \bar{\varphi}$. In this notation one has

$$
M=1+(S+\bar{S}) / 2, \quad \widetilde{M}=-1+(S+\bar{S}) / 2 .
$$

If we denote the dependence of the operator $S$ on the matrix $J$ by the notation $S=S(J)$, then $\overline{S(J)}=-S(\bar{J})$ (the minus sign appears due to the factor $1 / \pi i$ before the singular integral). According to Lemma 3.2, the operators $S(J)-S_{0}$ and $S(\bar{J})-S_{0}$ are compact in the space $L^{p}(\Gamma)$. But then the same property belongs to the operator $S+\bar{S}=S(J)-S(\bar{J})$. Therefore by the Riesz Theorem [15] the operators $M$ and $\widetilde{M}$ in (3.19) are Fredholm of index zero. In combination with (3.17), (3.18) this allows us to conclude that the operator $M$ is invertible and

$$
\operatorname{ker} \widetilde{M}=0, \quad \mathbb{R}^{l} \cap \operatorname{im} \widetilde{M}=0 .
$$

Now let $\phi \in H^{p}(D)$ and $f=\operatorname{Re} \phi^{+}$. Setting $\varphi=M^{-1} \varphi$, we obtain $(\phi-I \varphi)^{+}=0$ and by Lemma 3.2 the function $\phi=I \varphi+i \xi$ with some $\xi \in \mathbb{R}^{l}$. If in this equality $\phi=0$, then $M \varphi=0$ and hence $\varphi=0$. This proves the claim of the theorem in the case under consideration. 
Further let $\widetilde{\phi} \in H^{p}(\widetilde{D})$ and $\widetilde{\phi}_{0}(z)=\widetilde{\phi}(z)-\widetilde{\phi}(\infty)$. Recall that the operator $\widetilde{M}$ is Fredholm of index zero. Therefore, taking into account (3.20), the function $f=\operatorname{Re} \widetilde{\phi}_{0}$ can be represented in the form $\widetilde{M} \varphi+\xi$ with some $\varphi \in L^{p}$ and $\xi \in \mathbb{R}^{l}$. Then $\operatorname{Re}\left(\widetilde{\phi}_{0}-\widetilde{I} \varphi\right)=\xi$ and by Lemma 3.2 the function $\widetilde{\phi}_{0}-\widetilde{I} \varphi$ is constant. Since it vanishes at $\infty$, in fact one has $\widetilde{\phi}=\widetilde{I} \varphi$, which leads to the decomposition (3.15) for $\widetilde{\phi}$ with $\eta=\widetilde{\phi}(\infty)$. The fact that $\widetilde{\phi}=0$ in this decomposition implies $\eta=0$ and $\varphi \in \mathbb{R}^{l}$ can be proved similarly. Thus the claim of the Theorem is also proved for the case of an unbounded domain whose boundary is a simple contour.

Consider the general case of a contour $\Gamma$, assuming for definiteness that the domain $D$ is bounded. Let the domain $D_{j}$ have the contour $\Gamma_{j}$ as a boundary, being unbounded for $1 \leq j \leq m-1$ and bounded $j=m$, so that $D=D_{1} \cap \ldots \cap D_{m}$. According to the Cauchy Formula the function $\phi \in H^{p}(D)$ can be represented as the sum

$$
\phi(z)=\phi_{1}(z)+\ldots+\phi_{m}(z), \quad z \in D,
$$

where $\phi_{j} \in H^{p}\left(D_{j}\right)$ are defined by the integral of the Cauchy type

$$
\phi_{j}(z)=\frac{1}{2 \pi i} \int_{\Gamma_{j}}(t-z)_{J}^{-1} d t_{J} \phi^{+}(t), \quad z \in D_{j} .
$$

The conclusion of the theorem is already applicable to the functions $\phi_{j}$, so that

$$
\begin{gathered}
\phi_{j}(z)=\frac{1}{2 \pi i} \int_{\Gamma_{j}}(t-z)_{J}^{-1} d t_{J} \varphi_{j}(t), \quad 1 \leq j \leq m-1, \\
\phi_{m}(z)=\frac{1}{2 \pi i} \int_{\Gamma_{m}}(t-z)_{J}^{-1} d t_{J} \varphi_{m}(t)+i \xi, \quad \xi \in \mathbb{R}^{l} .
\end{gathered}
$$

Substituting these expressions into (3.21), we arrive at the required decomposition (3.15). If in this decomposition $\phi=0$ and the functions $\phi_{j} \in H^{p}\left(D_{j}\right)$ are defined by the Cauchy type integral with density $\left.\varphi\right|_{\Gamma_{j}}$, then the equality $-\phi_{m}=\phi_{1}+\ldots+\phi_{m-1}$ allows us to extend $-\phi_{m}$ up to a Douglis analytic function on the whole plane that vanishes at $\infty$. Therefore $\phi_{m}=0$. Similarly one can show that $\phi_{j}=0$ for all $j$. Applying the conclusion of the Theorem to $\phi_{j}$, we see that $\left.\varphi\right|_{\Gamma_{j}} \in \mathbb{R}^{l}$ for $1 \leq j \leq m-1$ and $\xi=0,\left.\varphi\right|_{\Gamma_{m}}=0$.

\section{The Riemann-Hilbert Problem}

As in the case of usual analytic functions, for Douglis analytic functions one can consider the Riemann-Hilbert Problem

$$
\left.\operatorname{Re} G \phi\right|_{\Gamma}=f
$$

where the $l \times l$-matrix function $G \in C(\Gamma)$ is invertible everywhere on $\Gamma$. This problem is considered in the space $H^{p}(D), p>1$, with a right-hand side $f \in L^{p}(\Gamma)$. The Fredholm property and the index of the problem are understood with respect to the $\mathbb{R}$-linear operator $\phi \rightarrow \operatorname{Re} G \phi$ of its boundary condition.

Theorem 4.1. Let the Lyapunov contour $\Gamma$ consist of $m$ connected components and the determinant of the matrix function $G \in C(\Gamma)$ be nonzero everywhere on $\Gamma$. Then problem (4.1) is Fredholm and its index $\varkappa$ is given by the formula

$$
\varkappa=-\left.\frac{1}{\pi} \arg \operatorname{det} G\right|_{\Gamma}+(2-m) l,
$$

where the increment of a continuous branch of the argument on $\Gamma$ is taken in the direction leaving the domain $D$ on the left. 
If $\Gamma \in C^{1, \nu}$ and $G \in C^{\nu}(\Gamma)$, then any solution $\phi \in H^{p}(D)$ of this problem with a right-hand side $f \in C^{\mu}(\Gamma), 0<\mu<\nu$, belongs to the class $C^{\mu}(\bar{D})$. Under the additional assumption $G \in C^{1, \nu}(\Gamma) a$ similar assertion also holds for the classes $C^{1, \mu}$.

Proof. Without loss of generality the matrix $J$ can be considered Jordan and, in particular, triangular. In fact, let a matrix $b \in \mathbb{C}^{l \times l}$ take $J$ to the Jordan form $J_{0}$, i.e., $J_{0}=b^{-1} J b$. Then the substitution $\phi=b \phi_{0}$ takes $J$-analytic functions to $J_{0}$-analytic ones. it remains to note that under this substitution problem (4.1) passes into a similar problem for a $J_{0}$-analytic function $\phi_{0}$ with a matrix $G_{0}=G b$.

Thus we can make use of Theorem 3.1. This theorem implies that the integral operator $I$ acting from the space $L^{p}(\Gamma)$ of real $l$-vector functions into $H^{p}(D)$ is Fredholm and its index ind $I=l(m-2)$. On the other hand, according to (3.7) for the composition $N=2 R I$ of the operator $R$ of problem (4.1) with $I$ we have the equality $N \varphi=\operatorname{Re}(\varphi+S \varphi)$. In terms of operator involution of conjugation introduced in the proof of Theorem 3.1 we can write

$$
N=G(1+S) / 2+\bar{G}(1+\bar{S}) / 2=G\left(1+S_{0}\right) / 2+\bar{G}\left(1-S_{0}\right) / 2+K
$$

with an integral operator $2 K=G\left(S-S_{0}\right)+\bar{G}\left(\bar{S}+S_{0}\right)$, which by Lemma 3.2 is compact in $L^{p}(\Gamma)$. According to the classical theory of singular operators with the Cauchy kernel $[9,13]$ we conclude that the operator $N$ is Fredholm in $L^{p}$ and its index is determined by the first term on the right-hand side of (4.2). Since $S_{0}^{2}=1$, with the help of Lemma 3.2 one can similarly check that the operator $N^{(-1)}=G^{-1}\left(1+S_{0}\right) / 2+\bar{G}^{-1}\left(1-S_{0}\right) / 2$ is a regularizer of $N$, i.e., the operators $1-N N^{(-1)}$ and $1-N^{(-1)} N$ are compact in $L^{p}(\Gamma)$.

The second part of the Theorem is based on Lemma 4.1, which will be proved below. Let $\Gamma \in$ $C^{1, \nu}, G \in C^{\nu}(\Gamma)$ and $0<\mu<\nu$. Then by Lemma 3.1 the operator $K$ in (4.4) is compact in the space $C^{\mu}(\Gamma)$ and the operator $N^{(-1)}$ is a regularizer of $N$ in this space. Therefore the claim of the theorem follows from Lemma 4.1.

Further let $G \in C^{1, \nu}(\Gamma)$. Consider the operation of differentiation on $\Gamma$ by the formula

$$
(D \varphi)\left(t_{0}\right)=\lim _{t \rightarrow t_{0}, t \in \Gamma}\left(t-t_{0}\right)_{J}^{-1}\left[\varphi(t)-\varphi\left(t_{0}\right)\right]
$$

It is related to the similar operation $D_{0}$ with respect to $J=i$ by the equality $D=d D_{0}$, where $d=e_{J}^{-1} e \in C^{\nu}(\Gamma)$. It can be directly checked by integration by parts that

$$
(I \varphi)^{\prime}(z)=\frac{1}{2 \pi i} \int_{\Gamma}(t-z)_{J}^{-1} d t_{J}(D \varphi)(t) .
$$

In particular, the Cauchy type operator $I$ is bounded $C^{1, \mu}(\Gamma) \rightarrow C^{1, \mu}(\bar{D})$. Therefore it suffices to establish the regularity statement of the theorem with respect to the equation $N \varphi=f$ defined by the operator (4.3).

Applying to the previous equality the Sohocki-Plemelj Formula (3.7) and comparing the result with the differentiated formula (3.7), we obtain the equality $D S=S D$ or $d D_{0} S=S d D_{0}$. Similarly one has $D_{0} S_{0}=S_{0} D_{0}$, so that

$$
D_{0}\left(S-S_{0}\right)=\left[d^{-1}\left(S-S_{0}\right) d+\left(d^{-1} S_{0}-S_{0} d^{-1}\right) d\right] D_{0} .
$$

As a result, for the operator $K$ in (4.3) we have the relation $D_{0} K=K_{0}+K_{1} D_{0}$, where $K_{j}(j=$ $0,1)$ are defined similarly to $(3.9)$ with some functions $k_{j}\left(t_{0}, t\right)$ having the property (3.8). Therefore the operator $K$ is compact in the space $C^{1, \mu}(\Gamma), \quad 0<\mu<\nu$. Similarly one can check that the operator $N^{(-1)}$ is a regularizer of $N$ in the space $C^{1, \mu}(\Gamma)$ as well, so that it remains to make use of Lemma 4.1.

Lemma 4.1. Let the operator $N$ be Fredholm in the space $L^{p}(\Gamma), \quad p>1$, and together with its regularizer $N^{(-1)}$ bounded in some Banach space $X$ embedded into $L^{p}(\Gamma)$. Then if $N^{(-1)}$ is a regularizer of $N$ with respect to $X$ as well, then any solution $\varphi \in L^{p}(\Gamma)$ of the equation $N \varphi=f$ with the right-hand side $f \in X$ also belongs to $X$. 
Proof. It suffices to prove the assertion with respect to the equation $\varphi+K \varphi=f$, where $K=$ $1-N^{(-1)} N$. By hypothesis, the operator $K$ is compact both in $L^{p}$ and in $X$. The space $L^{q}(\Gamma)$, $q=p /(p-1)$, is dual to $L^{p}(\Gamma)$ with respect to the bilinear form

$$
(\varphi, \psi)=\int_{\Gamma} \varphi(t) \psi(t) d t
$$

Respectively, the adjoint operator $K^{\prime}$ related to $K$ by the identity $(K \varphi, \psi)=\left(\varphi, K^{\prime} \psi\right)$ is compact in the space $L^{q}$. By the Riesz Theorem [15] the dimension of the kernel $n=\operatorname{dim}[\operatorname{ker}(1+K)]$ of the operator $1+K$ is finite and coincides with $\operatorname{dim}\left[\operatorname{ker}\left(1+K^{\prime}\right)\right]$, and the equation $N \varphi=f$ is solvable if and only if $(f, \psi)=0, \psi \in \operatorname{ker}\left(1+K^{\prime}\right)$. Let $n_{0}$ have an analogous sense with respect to the operator $K_{0}=K \mid X$ acting in $X$. Then, obviously, the condition $(f, \psi)=0, \psi \in \operatorname{ker}\left(1+K^{\prime}\right)$ is necessary for the solvability of the equation $N \varphi=f$ in $X$. Thus,

$$
n_{0} \leq n, \quad \operatorname{dim}\left[X / \operatorname{Im}\left(1+K_{0}\right)\right] \geq n_{0},
$$

so that $\operatorname{ind}\left(1+K_{0}\right) \leq \operatorname{ind}(1+K)=0$. Since in fact one has $\operatorname{ind}\left(1+K_{0}\right)=0$, the two previous inequalities are also exact equalities. Thus $\operatorname{ker}\left(1+K_{0}\right)=\operatorname{ker}(1+K)$, and the condition $(f, \psi)=$ $0, \psi \in \operatorname{ker}\left(1+K^{\prime}\right)$ is necessary and sufficient for the solvability of the equation $N \varphi=f$ in $X$. This directly implies the assertion of the lemma.

We pay a special attention to the Riemann-Hilbert problem with a constant matrix $G$. In this case the index formula (4.2) becomes $\varkappa=l(2-m)$. To consider this problem in more detail, it is convenient to introduce the Hardy space for the class of real vector functions

$$
u=\operatorname{Re} G \phi,
$$

which is denoted here by $h^{p}(D)$. It is introduced similarly to the case of $J$-analytic functions with the difference that the sequence of contours $\Gamma_{n} \subseteq D, n=1,2, \ldots$ converges to $\Gamma$ in the metric of this class. In other words, the domain $D$ is bounded by the contour $\Gamma \in C^{1, \nu}$ and condition (3.13) holds with respect to the norm of the space $C^{1, \nu}(\Gamma)$. Then the space $h^{p}(D)$ of functions $u=\operatorname{Re} G \phi$ is defined by the condition of finiteness of the norm

$$
|u|=\sup _{n}|u|_{L^{p}\left(\Gamma_{n}\right)} .
$$

Theorem 4.2. Let the domain $D$ be bounded by a contour $\Gamma$ of the class $C^{1, \nu}$; then $u \in h^{p}(D)$ if and only if in the representation (4.4) the function $\phi \in H^{p}(D)$.

Proof. Without loss of generality the matrix $J$ can be considered triangular, which is justified in the same way as in the proof of Theorem 4.1. Since the assertion of the theorem has to do with the behavior of $\phi$ near the connected components of the contour, we can consider the domain $D$ bounded, and the contour $\Gamma$ consisting of two components. In this case it is convenient to modify the operator $I$ of the Cauchy type integral slightly, putting

$$
(I \varphi)(z)=\frac{1}{2 \pi i}\left[\int_{\Gamma}(t-z)_{J}^{-1} d t_{J} \varphi(t)+\int_{\Gamma} \varphi(t)|d t|\right], \quad z \in D .
$$

Then by Theorem 3.1 this operator is invertible $L^{p}(\Gamma) \rightarrow H^{p}(D)$.

In our notation formula (3.7) corresponds to the equality

$$
2(I \varphi)^{+}=\varphi+S \varphi, \quad(S \varphi)\left(t_{0}\right)=\frac{1}{\pi i} \int_{\Gamma}\left[\left(t-t_{0}\right)_{J}^{-1} e_{J}(t)+1\right] \varphi(t)|d t|,
$$

and for the operator $N \varphi=\operatorname{Re} G(I \varphi)^{+}$we have an expression similar to (4.5):

$$
N=G(1+S) / 2+\bar{G}(1+\bar{S}) / 2 .
$$


Thus problem (4.1) is equivalent to the equation $N \varphi=2 f$, whose solution $\varphi$ determines the solution $\phi=I \varphi$ of the problem. According to Theorem 4.1 this problem and, respectively, the operator $N$ are Fredholm of index zero. Let $\phi_{1}, \ldots, \phi_{k} \in H^{p}(D)$ form the base of the space of solutions of the homogeneous problem (4.1). Without loss of generality we can consider that some subdomain $D_{0}$ together with its boundary lies inside all the contours $\Gamma_{n}$. Real $l$-vector functions $\operatorname{Re} \phi_{j}$ are linear independent as elements of $C\left(\overline{D_{0}}\right)$. In fact, if $\operatorname{Re} \phi \equiv 0$ in the domain $D_{0}$ for some $J$-analytic function $\phi$, then also $\phi \equiv 0$, which is proved similarly to Lemma 3.2. We choose a system of real $l$-vector functions $\psi_{1}, \ldots, \psi_{k}$, biorthogonal to functions $\operatorname{Re} \phi_{j}(z), z \in D_{0}$. In other words,

$$
\int_{D_{0}}\left(\operatorname{Re} \phi_{i}\right) \psi_{j} d x d y=\delta_{i j}
$$

where $\delta_{i j}$ is the Kronecker symbol. Then the homogeneous problem (4.1) complemented by the conditions

$$
\int_{D_{0}} \psi_{j} \operatorname{Re} \phi d x d y=0, \quad 1 \leq j \leq k,
$$

has only the zero solution. Consider the operator $L: L^{p}(\Gamma) \rightarrow \mathbb{R}^{k}$ defined by the formula

$$
(L \varphi)_{j}=\int_{D_{0}} \operatorname{Re} \psi_{j} \operatorname{Re}(I \varphi) d x d y, \quad 1 \leq j \leq k .
$$

Due to (4.6) it can be written in a more explicit form of the scalar product

$$
(L \varphi)_{j}=\int_{\Gamma} g_{j}(t) \varphi(t)|d t|, \quad 1 \leq j \leq k,
$$

with functions

$$
g_{j}(t)=\frac{1}{2 \pi} \int_{D_{0}} \operatorname{Im}\left[e_{J}(t)(t-z)_{J}^{-1}+1\right] \psi_{j}(z) d x d y .
$$

In this notation the operator $(N, L): L^{p}(\Gamma) \rightarrow L^{p}(\Gamma) \times \mathbb{R}^{k}$ is Fredholm and its kernel is trivial. The dependence of operators (4.7)-(4.9) and their defining functions on $\Gamma$ is indicated by subscripts $S_{\Gamma}, L_{\Gamma}$, etc.

We turn to the sequence of contours $\Gamma_{n}$ considered in the Theorem. By hypothesis, there exist homeomorphisms $\gamma_{n}: \Gamma \rightarrow \Gamma_{n}$ of the class $C^{1, \nu}(\Gamma)$ such that condition (3.12) holds in the norm of $C^{1, \nu}$, i.e.,

$$
\lim _{n \rightarrow \infty}\left|\gamma_{n}(t)-t\right|_{C^{1, \nu}}=0 .
$$

The operation of superposition $\varphi \rightarrow \varphi \circ \gamma_{n}$ of functions induces the operator transformation $M \rightarrow$ $M \circ \alpha_{n}$ by the rule $\left(M \circ \gamma_{n}\right)\left(\varphi \circ \gamma_{n}\right)=(M \varphi) \circ \gamma_{n}$, which takes the Banach space $\mathcal{L}\left[L^{p}\left(\Gamma_{n}\right)\right]$ of bounded operators in $L^{p}\left(\Gamma_{n}\right)$ into $\mathcal{L}\left[L^{p}(\Gamma)\right]$. The notation $\left(M \circ \gamma_{n}\right)\left(\varphi \circ \gamma_{n}\right)=M \varphi$ for the operator $M: L^{p}\left(\Gamma_{n}\right) \rightarrow \mathbb{R}^{k}$ has a similar sense. We assert that in this notation

$$
\left|S_{\Gamma_{n}} \circ \gamma_{n}-S_{\Gamma}\right|_{\mathcal{L}} \rightarrow 0, \quad\left|L_{\Gamma_{n}} \circ \gamma_{n}-L_{\Gamma}\right|_{\mathcal{L}} \rightarrow 0
$$

as $n \rightarrow \infty$ in the operator norm of the respective spaces. In fact, set

$$
q_{n}\left(t_{0}, t\right)=\left[\gamma_{n}(t)-\gamma_{n}\left(t_{0}\right)\right]_{J}^{-1} e_{J}\left[\gamma_{n}(t)\right] e_{J}^{-1}(t)\left(t-t_{0}\right)_{J} .
$$

Then by (4.10) the sequence of matrix functions $q_{n} \rightarrow 1$ in the norm of $C^{\nu}(\Gamma \times \Gamma)$. It remains to note that in this notation one has

$$
\left[\left(S \circ \gamma_{n}\right) \varphi\right]\left(t_{0}\right)=\frac{1}{\pi i} \int_{\Gamma}\left[q_{n}\left(t_{0}, t\right)\left(t-t_{0}\right)_{J}^{-1} e_{J}(t)+1\right] \varphi(t)\left|\gamma_{n}^{\prime}(t) \| d t\right|,
$$




$$
\left[\left(L_{\Gamma_{n}} \circ \gamma_{n}\right) \varphi\right]_{j}=\int_{\Gamma} g_{\Gamma_{n}, j}\left[\gamma_{n}(t)\right]\left|\gamma_{n}^{\prime}(t)\right| \varphi(t)|d t|,
$$

and to make use of the estimate of he operator norm of the operator $K$ in $L^{p}(\Gamma)$ established in the proof of Lemma 3.1.

Now let a $J$-analytic in $D$ function $\phi$ be given, for which norm (4.5) is finite, i.e., real functions $f_{n}=\left.\operatorname{Re} \phi\right|_{\Gamma_{n}}$ are uniformly bounded in the norm of the spaces $L^{p}\left(\Gamma_{n}\right)$. Put

$$
\xi_{j}=\int_{D_{0}} \psi_{j} \operatorname{Re} \phi d x d y, \quad 1 \leq j \leq k .
$$

Let $\varphi_{n} \in L^{p}\left(\Gamma_{n}\right)$ be defined by the equality $\phi=I_{\Gamma_{n}} \varphi_{n}$ in the domain $D_{n} \subseteq D$ bounded by the contour $\Gamma_{n}$. Then one has $N_{\Gamma_{n}} \varphi_{n}=2 f_{n}$ and $\left(L_{\Gamma_{n}} \varphi_{n}\right)_{j}=\xi_{j}$, or, equivalently,

$$
\left(N_{\Gamma_{n}} \circ \alpha_{n}\right) \widetilde{\varphi}_{n}=2 f_{n}, \quad\left[\left(L_{\Gamma_{n}} \circ \alpha_{n}\right) \widetilde{\varphi}_{n}\right]_{j}=\xi_{j},
$$

where we put $\widetilde{\varphi}_{n}=\varphi_{n} \circ \alpha_{n}$. According to (4.8) relation (4.11) also holds for the operator $N$; therefore by Lemma 4.2 below the sequence $\widetilde{\varphi}_{n}$ is bounded in $L^{p}(\Gamma)$. Due to $(4.7),(4.11)$ this implies that the sequence of functions $\left(S_{\Gamma_{n}} \circ \alpha_{n}\right) \widetilde{\varphi}_{n}$ and hence also $\phi \circ \alpha_{n}$ are bounded in $L^{p}(\Gamma)$, which completes the proof of the theorem.

Lemma 4.2. Let Banach spaces $X, Y$ and the Fredholm operator $N \in \mathcal{L}(X, Y)$ with a trivial kernel be given. Let the sequence $N_{n} \rightarrow N$ as $n \rightarrow \infty$ in the norm of the space $\mathcal{L}(X, Y)$. Then, if the sequence of vectors $N_{n} x_{n}$ is bounded in $Y$, so are $x_{n}$ in $X$.

Proof. First suppose that the image $\operatorname{im} N$ of the operator coincides with $Y$, i.e., the operator $N$ is invertible. Then the operators $N_{n}$ are also invertible for sufficiently large $n$ and the sequence $N_{n}^{-1}$ converges to $N^{-1}$ in $\mathcal{L}(Y, X)$ so that the sequence $x_{n}=N_{n}^{-1} y_{n}$ is bounded. In the general case by hypothesis the image $\operatorname{im} N$ is closed and $Y=Y_{0} \oplus \operatorname{im} N$ for some finite-dimensional subspace $Y_{0}$. Consider the operators $\widetilde{N}, \widetilde{N}_{n} \in \mathcal{L}\left(X \times Y_{0}, Y\right)$ defined by the formula

$$
\widetilde{N}\left(x, y_{0}\right)=N x+y_{0}, \quad \widetilde{N}_{n}\left(x, y_{0}\right)=N_{n} x+y_{0} .
$$

Then the operator $\widetilde{N}$ is invertible and the sequence $\widetilde{N}_{n} \rightarrow \widetilde{N}$ in the operator norm. Since $\widetilde{N}_{n}\left(x_{n}, 0\right)=$ $y_{n}$, this implies the boundedness of the sequence $x_{n}$.

Note that an analogue of Theorem 4.2 also holds with respect to the Hölder classes $C^{\mu}(\bar{D}), 0<$ $\mu<\nu$.

To conclude, we discuss multivalued $J$-analytic functions, i.e., functions $\phi$ with single-valued derivatives $\phi^{\prime}$ in the domain $D$. Here in the case of an unbounded domain it is assumed that this derivative is of the order -2 at $\infty$. These functions can be easily reduced to single-valued ones with the help of a special matrix function $L(z)$ such that its derivative coincides with $(2 \pi i)^{-1} z_{J}^{-1}$. It is easy to show [17] that it can be defined as a value of the analytic function $(2 \pi i)^{-1} \ln \zeta$ of the matrix $z_{J}$. Then for any $\eta \in \mathbb{C}^{l}$ the multivalued vector function $\phi(z)=L(z) \eta$ will be a $J$-analytic function such that its increment in the anti-clockwise motion of the point $z=0$ yields the vector $\eta$. In the case of matrix $(2.1)$ this function is given by the formula

$$
\begin{aligned}
& \text { (i) } L(z)=\frac{1}{2 \pi i}\left(\begin{array}{cc}
\ln \left(x+\nu_{1} y\right) & 0 \\
0 & \ln \left(x+\nu_{2} y\right)
\end{array}\right), \\
& \text { (ii) } L(z)=\frac{1}{2 \pi i}\left(\begin{array}{cc}
\ln (x+\nu y) & y(x+\nu y)^{-1} \\
0 & \ln (x+\nu y)
\end{array}\right),
\end{aligned}
$$

Starting from $L(z)$ construct a family of multivalued functions with a similar behavior with respect to the connected components of the contour $\Gamma=\partial D$. Let this contour consist of connected components $\Gamma_{1}, \ldots, \Gamma_{m}$, and let in the case of a bounded domain $D$ the contour $\Gamma_{m}$ encompass all the other ones. 
Evidently, the complement of the closed domain $\bar{D}$ on the plane consists of $m$ domains $\widetilde{D}_{1}, \ldots, \widetilde{D}_{m}$ such that each of them has a simple contour as its boundary. For $1 \leq j \leq m-1$ all the domains $\widetilde{D}_{j}$ are bounded, and the domains $\widetilde{D}_{m}$ and $D$ are of the opposite type.

Choose inside each domain $\widetilde{D}_{j}$ a point $a_{j}$ and put

$$
L_{j}(z)=\frac{1}{2 \pi i}\left\{\begin{array}{ll}
L\left(z-a_{j}\right), & \sigma(D)=1, \\
L\left(z-a_{j}\right)-L\left(z-a_{m}\right), & \sigma(D)=0,
\end{array} \quad 1 \leq j \leq m-1 .\right.
$$

Note that in the case of an unbounded domain the derivative $L_{j}^{\prime}(z)$ is of the order -2 at $\infty$.

It is easy to see that in this notation each multivalued function is representable in the form

$$
\phi(z)=\phi_{0}(z)+\sum_{j=1}^{m-1} L_{j}(z) \eta_{j}, \quad \eta_{j} \in \mathbb{C}^{l},
$$

where $\phi_{0}$ is single-valued. According to this, the notation $\phi \in h^{p}(D)$ means by definition that in this representation one has $\phi_{0} \in h^{p}(D)$. In a similar way, other classes of multivalued functions are introduced.

The formulation of the Riemann-Hilbert problem (4.1) can be extended to admissible multivalued functions, i.e., functions $\phi$ such that the real $l$-vector function $\operatorname{Re} G \phi$ is single-valued. They are defined by decomposition (4.14), where $\operatorname{Re} G \eta_{j}=0$. Accordingly, this problem can be considered as

$$
\left.\operatorname{Re} G \phi_{0}\right|_{\Gamma}+\left.\sum_{j=1}^{m-1} \operatorname{Re}\left[G L_{j} \eta_{j}\right]\right|_{\Gamma}=f
$$

with respect to $\left(\phi_{0}, \eta_{1}, \ldots, \eta_{m-1}\right)$, where $\phi_{0}$ is single-valued, and the vectors $\eta_{j}(j=1, \ldots, m-1)$ belong to the finite-dimensional space $\left\{\eta \in \mathbb{C}^{l}, \operatorname{Re} G \eta=0\right\}$ of dimension $l$. It is well known [14] that extension of a Fredholm operator by $n$ dimensions increases its index by $n$. Therefore due to (4.2) the index of problem (4.1) in the class $h^{p}(D)$ of admissible multivalued functions equals $l$.

\section{First and Second Boundary-Value Problem for the Lamé System}

Consider the Lamé system in a domain $D$ bounded by a Lyapunov contour $\Gamma \in C^{1, \nu}, 0<\nu<1$. It is known [12] that the main boundary conditions for this system consist in fixing on the boundary contour either the shift vector

$$
u^{+}=f
$$

or the normal component $\sigma^{+} n=\sigma_{(1)}^{+} n_{1}+\sigma_{(2)}^{+} n_{2}$ of the stress tensor $\sigma$, where $n=\left(n_{1}, n_{2}\right)$ is the exterior unit normal on $\Gamma$ and the superscript + denotes the boundary value of the function.

According to (1.1), (2.13) the latter boundary condition can be written in the form

$$
n_{1}\left(a_{11} \frac{\partial u}{\partial x}+a_{12} \frac{\partial u}{\partial y}\right)^{+}+n_{2}\left(a_{21} \frac{\partial u}{\partial x}+a_{22} \frac{\partial u}{\partial y}\right)^{+}=g
$$

where we put $x_{1}=x, x_{2}=y$. Thus (5.1) corresponds to the Dirichlet problem for the Lamé system, and (5.2) to the Neumann problem. These problems are also called the first and the second boundary value problems, respectively.

In the case $\sigma(D)=0$ of an unbounded domain the following condition is imposed on the gradient of the solution $u$ of this system:

$$
\operatorname{grad} u(z)=O\left(|z|^{-2}\right) \quad \text { as } \quad z \rightarrow \infty,
$$

in particular, there exists a limit $u(\infty)=\lim u(z)$ at infinity. Then Theorem 2.1 implies that in representation (2.10) function $\phi^{\prime}(z)$ is of the order -2 at $\infty$.

From the viewpoint of general strongly elliptic systems the solvability of the Dirichlet and Neumann problems for the Lamé system in Hölder and Sobolev spaces is well studied [6]. In this section we 
consider these problems in Hardy classes $h^{p}(D)$ for the solutions of the Lamé system and the conjugate functions, which according to Theorems 2.1, 2.2 are defined as in Section 4 with respect to $G=b$ and $G=c$, respectively.

In the class $C^{1}(\bar{D})$ the uniqueness of the solution of the Dirichlet problem for system (1.1) easily follows from Green's Formula:

$$
\sum_{i, j=1}^{2} \int_{D}\left(a_{i j} \frac{\partial u}{\partial x_{j}}\right) \frac{\partial u}{\partial x_{i}} d x_{1} d x_{2}=\sum_{i, j=1}^{2} \int_{\Gamma}\left(a_{i j} \frac{\partial u}{\partial x_{j}} n_{i}\right) u|d t|,
$$

where we put for the sake of uniformity $x_{1}=x, x_{2}=y$. If $u$ satisfies the homogeneous boundary condition (5.1), then the integral over $\Gamma$ falls out of this equality, and due to (1.4) the solution $u$ must be trivial, i.e., of the form (2.15). Combining this with (1.4) yields $u=0$.

For the same reasons we conclude that the homogeneous problem (5.2) admits only trivial solutions. On the other hand, for two solutions $u, u_{0} \in C^{1}(\bar{D})$, similarly to (5.4), we can write the identity

$$
\sum_{i, j=1}^{2} \int_{D}\left(a_{i j} \frac{\partial u}{\partial x_{j}}\right) \frac{\partial u_{0}}{\partial x_{i}} d x_{1} d x_{2}=\sum_{i, j=1}^{2} \int_{\Gamma}\left(a_{i j} \frac{\partial u}{\partial x_{j}} n_{i}\right) u_{0}|d t| .
$$

If $u_{0}$ is a trivial solution, then

$$
\sum_{i, j=1}^{2} \int_{D} a_{i j} \frac{\partial u}{\partial x_{j}} \frac{\partial u_{0}}{\partial x_{i}} d x_{1} d x_{2}=\sum_{i, j=1}^{2} \int_{D} a_{i j} \frac{\partial u_{0}}{\partial x_{j}} \frac{\partial u}{\partial x_{i}} d x_{1} d x_{2}=0
$$

so that the condition

$$
\int_{\Gamma} g(t) u_{0}(t)|d t|=0
$$

of orthogonality of the right-hand side of problem (5.2) to the trivial solutions $u_{0}$ is necessary for its solvability.

Theorem 5.1. Let the domain $D$ be bounded by a contour $\Gamma \in C^{1, \nu}$. Then the Dirichlet problem is uniquely solvable in the class $h^{p}(D), p>1$.

If the right-hand side $f \in C^{\mu}(\Gamma), 0<\mu<\nu$, then any solution $u \in h^{p}(D)$ of this problem belongs to $C^{\mu}(\bar{D})$. Similarly, $f \in C^{1, \mu}(\Gamma)$ implies $u \in C^{1, \mu}(\bar{D})$.

Proof. By Theorems 2.1 and 3.3 the Dirichlet problem is equivalent to the Riemann-Hilbert one

$$
\operatorname{Re} b \phi^{+}=f
$$

in the class $h^{p}(D)$ of admissible multivalued solutions. As we have noted at the end of Section 3, this problem is Fredholm in the class under consideration and its index equals 2. If $f=0$, then by Theorem 4.1 the function $\phi \in C^{1}(\bar{D})$ and, respectively, $u=\operatorname{Re} b \phi$ is a solution of the homogeneous Dirichlet problem for the Lamé system. As it was noted above, we have $u=0$, so that by Theorem 2.1 the function $\phi$ is constant. So, the dimension of the solution space of the homogeneous problem (5.6) equals 2 and, since its index equals 2 , this problem is unconditionally solvable. Thus we have established the first assertion of the theorem. The second part of the theorem is a corollary of the second part of Theorem 4.1.

We turn to the formulation of problem (5.2) in the Hardy class. If $u \in C^{1}(\bar{D})$, then by (2.14) equality (5.2) can be written with respect to the conjugate function $v$ in the form

$$
\left(v^{+}\right)^{\prime}=g,
$$

where the prime denotes the derivative in the arc length parameter measured in the positive direction with respect to $D$. 
Let $\Gamma_{j}, \quad 1 \leq j \leq m$, be simple contours that make $\Gamma$. By $(2.19)$, there exist unique $\eta_{j} \in \mathbb{C}^{2}$ that satisfy the system

$$
\operatorname{Re} b \eta_{j}=0, \operatorname{Re} c \eta_{j}=\int_{\Gamma_{j}} g(t)|d t|, \quad 1 \leq j \leq m-1 .
$$

Consider the multivalued solution of the Lamé system

$$
u_{1}(z)=u(z)-\operatorname{Re} \sum_{j=1}^{m-1} b L_{j}(z) \eta_{j}
$$

such that its conjugate function by Theorem 2.2 is given by the equality

$$
v_{1}(z)=v(z)-\operatorname{Re} \sum_{j=1}^{m-1} c L_{j}(z) \eta_{j} .
$$

Evidently, it satisfies a boundary condition similar to (5.7):

$$
\left(v_{1}^{+}\right)^{\prime}=g_{1}
$$

with the right-hand side

$$
g_{1}=g-\left[\left(\sum_{j=1}^{m-1} \operatorname{Re} c L_{j} \eta_{j}\right)^{+}\right]^{\prime}
$$

By (5.8), the function $g_{1}$ satisfies the condition

$$
\int_{\Gamma_{j}} g_{1}(t)|d t|=0,1 \leq j \leq m,
$$

Let the necessary condition (5.5) of solvability of the Neumann problem hold. Then, choosing as $u_{0}$ constant vector functions, we arrive at an equality similar to (5.11) on $\Gamma_{m}$ as well. Thus there exists a primitive $f_{1} \in C^{1}(\Gamma)$ of the function $g_{1}$. Here the function $v_{1}$ is single-valued, and (5.9) becomes the Dirichlet problem

for the conjugate function $v$.

$$
v_{1}^{+}=f_{1}
$$

This problem can already be considered both in the Hölder class $C^{\mu}(\bar{D})$ and in the Hardy class $h^{p}(D)$ of conjugate functions. If a single-valued conjugate function $v_{1} \in h^{p}(D)$ of the solution $u_{1}$ of the Lamé system satisfying the boundary condition (5.12) is found, then we will call the single-valued function $u$ defined by equality (5.9) the solution of the Neumann problem. The described procedure establishes a correspondence between problem (5.2) of solving the Lamé system with a multivalued conjugate function and problem (5.12) for single-valued conjugate functions to multivalued solutions of the Lamé system.

In the case $v_{1} \in C^{1, \mu}(\bar{D})$ the function $u$ defined in such a way belongs to the same class and satisfies the classical boundary condition (5.2). In particular, then condition (5.5) must necessarily hold for $g_{1}$, since it takes place for $g$ and for the restriction of the function $\sum \operatorname{Re} c L_{j} \eta_{j}$ to $\Gamma$. Recall that in the case of an unbounded domain only constant vectors are trivial solutions.

Thus after integration by parts the orthogonality condition (5.5) in the case of an unbounded domain disappears, and in the case of a bounded domain $D$ it becomes a single condition

$$
\int_{\Gamma} f_{1}(t) n(t)|d t|=0
$$

where the integrand is understood as the scalar product of $f_{1}(t)$ and the normal vector $n=\left(n_{1}, n_{2}\right)$ at the point $t \in \Gamma$ in $\mathbb{R}^{2}$. 
So, if the domain $D$ is bounded and $f_{1} \in C^{\mu}(\Gamma)$, then condition (5.13) is necessary for the solvability of problem (5.12). By density, we conclude that it is necessary for any right-hand side $f_{1} \in L^{p}(\Gamma)$.

Theorem 5.2. Let the domain $D$ be bounded by a contour $\Gamma \in C^{1, \nu}$. Then the homogeneous Dirichlet problem for conjugate functions in the class $h^{p}(D), p>1$, has only the zero solution, and the inhomogeneous problem is unconditionally solvable in the case $\sigma(D)=0$, and for $\sigma(D)=1$ it is solvable if and only if its right-hand side satisfies the orthogonality condition (5.13). In particular, this problem is Fredholm and its index equals $-\sigma(D)$.

If the right-hand side $f_{1} \in C^{\mu}(\Gamma), \quad 0<\mu<\nu$, then any solution $v_{1} \in h^{p}(D)$ of this problem belongs to $C^{\mu}(\bar{D})$. Similarly, $f_{1} \in C^{1, \mu}(\Gamma)$ implies $v_{1} \in C^{1, \mu}(\bar{D})$.

Proof. It is similar to that of Theorem 5.1. By Theorems 2.2 and 4.1 the Dirichlet problem (5.12) is equivalent to the Riemann-Hilbert one

$$
\operatorname{Re} c \phi^{+}=f_{1}
$$

in the class $h^{p}(D)$ of admissible multivalued solutions. In particular, by Theorem 4.1 the latter problem is Fredholm with index 2. This also implies the second part of the theorem, in particular, any solution $v$ of the homogeneous problem belongs to the class $C^{1, \mu}(\bar{D})$. The multivalued solution $u$ of the Lamé system such that its conjugate function is $v$ has a similar property as well.

Let us call a smooth arc joining distinct contours $\Gamma_{j}$ a $c u t$ of the domain $D$. Consider finitely many nonintersecting cuts $L_{1}, \ldots, L_{n}$ that divide the domain $D$ into subdomains $D_{1}, \ldots, D_{l}$ such that each of them is bounded by a simple piecewise smooth contour. Then in each domain $D_{k}$ any multivalued $J$-analytic function $\phi$ admits a single-valued branch $\phi_{k}$, and if $L_{s} \subseteq \partial D_{k} \cap \partial D_{r}$, then the difference $\phi_{k}-\phi_{r}$ is constant in $L_{s}$.

Now consider a multivalued solution $\phi$ of the homogeneous problem (5.14), which by Theorem 4.1 belongs to the class $C^{1}(\bar{D})$. By definition, for this solution the function $v=\operatorname{Re} c \phi$ is single-valued in the domain $D$ and vanishes on $\Gamma$. Then we can apply identity (5.4) to functions $u_{r}=\operatorname{Re} b \phi_{r}$ in the domain $D_{k}$ :

$$
\sum_{i, j=1}^{2} \int_{D_{k}}\left(a_{i j} \frac{\partial u_{k}}{\partial x_{j}}\right) \frac{\partial u_{k}}{\partial x_{i}} d x_{1} d x_{2}=\int_{\partial D_{k}} v_{k}^{\prime} u_{k}|d t|,
$$

where $v_{k}=\operatorname{Re} c \phi_{k}$ and the prime denotes the tangential derivative in the direction leaving the domain $D_{k}$ on the left. The sum of the right-hand sides of this equality for all $k$ 's is zero. In fact, the integrals over $\Gamma \cap \partial D_{k}$ are zero due to the boundary condition for $v$. On the other hand, if $L_{s} \subseteq \partial D_{k} \cap \partial D_{r}$, then on $L_{s}$ we have equalities $v_{k}=v_{r}$ and $v_{k}^{\prime}=-v_{r}^{\prime}$, since the tangential derivatives are taken in the opposite directions. On the other hand, the difference $u_{k}-u_{r}$ on $L_{s}$ equals the constant vector $\xi_{s} \in \mathbb{R}^{2}$. Therefore up to the sign

$$
\int_{\partial D_{k}} v_{k}^{\prime} u_{k}|d t|+\int_{\partial D_{r}} v_{r}^{\prime} u_{k}|d t|=\int_{\partial D_{k}} v_{k}^{\prime} \xi_{s}|d t|=0,
$$

where we have taken into account the fact that the function $v_{k}$ vanishes at the ends of $L_{s}$.

Thus the integral of the left-hand side of (5.4) equals zero and, respectively, $u=\operatorname{Re} b \phi$ is a trivial solution of the Lamé system and $v=0$.

As it was noted in Section 2, the functions $\phi$ with this property in the case $\sigma(D)=1$ are polynomials of the first degree and form a three-dimensional space, and for $\sigma(D)=1$ they are constant vectors and form a two-dimensional space. Since the index of the problem equals 2 , this implies that in the case $\sigma(D)=1$ the inhomogeneous problem is unconditionally solvable, and for $\sigma(D)=0$ this problem is solvable if one orthogonality condition holds, which is obviously (5.13).

As a corollary of Theorem 5.2 we obtain the following classical result on the solvability of the Neumann problem. 
$h^{p}(D), p>1$, has only the zero solution, and the inhomogeneous problem is unconditionally solvable in the case $\sigma(D)=0$, and for $\sigma(D)=1$ it is solvable if and only if its right-hand side satisfies the orthogonality condition (5.13). In particular, this problem is Fredholm and its index equals $-\sigma(D)$.

Theorem 5.3. Let the domain $D$ be bounded by a contour $\Gamma \in C^{1, \nu}$. Then the homogeneous Neumann problem for the Lamé system in the class $C^{1, \mu}(\bar{D}), 0<\mu<\nu$, has only trivial solutions, and the inhomogeneous problem is solvable if and only if its right-hand side satisfies the orthogonality condition (5.15) to these trivial solutions.

We also illustrate the application of Theorem 5.2 in the following situation.

Lemma 5.1. Let the domain $D$ be unbounded and have a simple contour $\Gamma \in C^{1, \nu}$ as its boundary. Let $f$ be the restriction of the polynomial $p(x, y)=x \xi^{0}+y \xi^{1}$ to $\Gamma$, where $\xi^{k}(k=0,1)$ appear in $(2.30)$. Then there exists a unique $J$-analytic function $\phi_{0}$, which belongs to the class $C^{1, \mu}(\bar{D})$ for any $\mu<\nu$ vanishes at infinity and satisfies the boundary condition

$$
\operatorname{Re} c \phi_{0}^{+}=\xi+f_{1}
$$

with some $\xi \in \mathbb{R}^{2}$.

Proof. It requires only uniqueness. Let the function $\phi \in H^{p}(D)$ vanish at infinity and satisfy the boundary condition $\operatorname{Re} c \phi^{+}=\xi$ for some $\xi \in \mathbb{R}^{2}$. Then by Theorem 5.2 the function $v=\operatorname{Re} c \phi$ is the identical zero, so that by Theorem 4.2 the function $\phi$ is a polynomial of the second degree. Since by hypothesis $\phi(z) \rightarrow 0$ as $z \rightarrow \infty$, this polynomial equals zero. Thus we establish the uniqueness of the function $\phi_{0}$ from the Lemma.

\section{Double Layer Potentials}

Consider in the domain $D$ integrals

$$
\begin{aligned}
& (P \varphi)(z)=\frac{1}{\pi} \int_{\Gamma} \frac{\operatorname{Re}[\overline{n(t)}(t-z)]}{|t-z|^{2}} \varphi(t)|d t|, \quad z \in D, \\
& (Q \varphi)(z)=\frac{1}{\pi} \int_{\Gamma} \frac{\operatorname{Im}[\overline{n(t)}(t-z)]}{|t-z|^{2}} \varphi(t)|d t| \quad z \in D,
\end{aligned}
$$

with real density $\varphi$, where $n=n_{1}+i n_{2}$ is the exterior unit normal on the Lyapunov contour $\Gamma$.

Integrals (6.1) and (6.2) can also be considered for $z=t_{0} \in \Gamma$. In this case we denote them by $P^{*} \varphi$ and $Q^{*} \varphi$, respectively. Since $\Gamma$ is a Lyapunov contour, similarly to Lemma 3.1 one can check that the kernel of the integral operator $P$ has a weak singularity. As for integral (6.2), it is singular, like the respective Cauchy integral.

Since $d t=i n|d t|$, these integrals are related to the Cauchy type integral by the formula

$$
(P \varphi)(z)-i(Q \varphi)(z)=\frac{1}{\pi i} \int_{\Gamma} \frac{\varphi(t) d t}{t-z} .
$$

Therefore the functions $P \varphi$ and $Q \varphi$ are harmonic in the domain $D$. Equality (6.3) immediately implies that the operators $P$ and $Q$ are bounded $L^{p}(\Gamma) \rightarrow h^{p}(D), p>1$, and the formulas

$$
(P \varphi)^{+}=\varphi+P^{*} \varphi, \quad(Q \varphi)^{+}=Q^{*} \varphi
$$

for their boundary values hold. For the same reasons under assumption $\Gamma \in C^{1, \nu}$ these operators are bounded $C^{\mu}(\Gamma) \rightarrow C^{\mu}(\bar{D})$ and $C^{1, \mu}(\Gamma) \rightarrow C^{1, \mu}(\bar{D}), 0<\mu<\nu$. It is also well known that the operator $P$ is bounded $C(\Gamma) \rightarrow C(\bar{D})$.

Integral (6.1) is a classical double layer potential. We construct generalized double layer potentials for the solutions of the Lamé system and their conjugate functions by the same scheme starting from matrix (2.1), a Cauchy type integral for $J$-analytic functions and the matrices $b, c$ that appear in 
Theorems 2.1 and 2.2. For this purpose, in notation (3.1) we introduce homogeneous matrix functions of order -1 of the variable $\xi=\xi_{1}+i \xi_{2}$ by the formulas

$$
\begin{array}{ll}
H_{11}(\xi)=\operatorname{Im}\left[b(i \xi)_{J} \xi_{J}^{-1} b^{-1}\right], & H_{22}(\xi)=\operatorname{Im}\left[c(i \xi)_{J} \xi_{J}^{-1} c^{-1}\right], \\
H_{21}(\xi)=\operatorname{Im}\left[c(i \xi)_{J} \xi_{J}^{-1} b^{-1}\right], & H_{12}(\xi)=\operatorname{Im}\left[b(i \xi)_{J} \xi_{J}^{-1} c^{-1}\right] .
\end{array}
$$

Note that in the explicit form $(i \xi)_{J} \xi_{J}^{-1}=\left(-\xi_{2}+\xi_{1} J\right)\left(\xi_{1}+\xi_{2} J\right)^{-1}$. Evidently, the matrices $H(\xi)$ are homogeneous of order zero and by Lemma 2.1 do not depend on the choice of $b$. These matrices define the integral operators

$$
\begin{aligned}
& \left(P_{k r} \varphi\right)(z)=\frac{1}{\pi} \int_{\Gamma} \frac{\operatorname{Re}[\overline{n(t)}(t-z)]}{|t-z|^{2}} H_{k r}(t-z) \varphi(t)|d t|, \quad z \in D, \\
& \left(P_{k r}^{*} \varphi\right)(z)=\frac{1}{\pi} \int_{\Gamma} \frac{\operatorname{Re}\left[\overline{n(t)}\left(t-t_{0}\right)\right]}{\left|t-t_{0}\right|^{2}} H_{k r}\left(t-t_{0}\right) \varphi(t)|d t|, \quad t_{0} \in \Gamma .
\end{aligned}
$$

As in the case of $P^{*}$, the kernel of the operators $P_{k r}^{*}$ has a weak singularity, which due to the evenness of the matrix functions $H(\xi)$ can be established in a way completely similar to the proof of Lemma 3.2. Therefore by Lemma 3.1 the operators $P_{k r}^{*}$ are compact in $L^{p}(\Gamma)$.

The next lemma describes the relation of the functions $P_{k r} \varphi$ with the Cauchy type integral $I \varphi$ introduced in Section 3. It is convenient to introduce the class $h^{p}$ for functions $w=P_{k r} \varphi$ by the condition of finiteness of the respective expression on the right-hand side of (3.8).

Lemma 6.1. The following equality holds:

$$
\left[\operatorname{Im}\left(b_{k} b_{r}^{-1}\right)\right] Q \varphi+P_{k r} \varphi=2 \operatorname{Re}\left[b_{k} I\left(b_{r}^{-1} \varphi\right)\right], \quad k, r=1,2,
$$

where, for the sake of uniformity, we put $b_{1}=b, b_{2}=c$. In particular, the operators $P_{k r}$ are bounded $L^{p}(\Gamma) \rightarrow h^{p}(D), p>1$, and the following formulas for angular boundary values hold:

$$
\begin{aligned}
& \left(P_{k k} \varphi\right)^{+}=\varphi+P_{k k}^{*} \varphi, k=1,2, \\
& \left(P_{21} \varphi\right)^{+}=\left[\operatorname{Re}\left(c b^{-1}\right)\right] \varphi+P_{21}^{*} \varphi \\
& \left(P_{12} \varphi\right)^{+}=\left[\operatorname{Re}\left(b c^{-1}\right)\right] \varphi+P_{12}^{*} \varphi .
\end{aligned}
$$

Proof. In terms of the tangential unit vector $e=i n$ one can write $e \xi^{-1}=|\xi|^{-2}[\operatorname{Re}(\bar{n} \xi)-i \operatorname{Im}(\bar{n} \xi)]$ or

$$
|\xi|^{2}\left(e_{1}+i e_{2}\right)=[\operatorname{Im}(\bar{n} \xi)] \xi+[\operatorname{Re}(\bar{n} \xi)] i \xi .
$$

Since the expressions in square brackets are real, this yields

$$
|\xi|^{2} e_{J}=[\operatorname{Im}(\bar{n} \xi)] \xi_{J}+[\operatorname{Re}(\bar{n} \xi)](i \xi)_{J} .
$$

which due to definition (6.5) leads to the equality

$$
\left.|\xi|^{2} \operatorname{Im}\left[b_{k} \xi_{J}^{-1} e_{J}\right) b_{r}^{-1}\right]=[\operatorname{Im}(\bar{n} \xi)] \operatorname{Im}\left(b_{k} b_{r}^{-1}\right)+[\operatorname{Re}(\bar{n} \xi)] H_{k r}(\xi) .
$$

By (6.2), (6.6) this implies (6.8).

A similar equality holds for the integral operators with a "star" superscript:

$$
\left[\operatorname{Im}\left(b_{k} b_{r}^{-1}\right)\right] Q_{0}^{*} \varphi+P_{k r}^{*} \varphi=\operatorname{Re}\left[b_{k} S\left(b_{r}^{-1} \varphi\right)\right] .
$$

On the other hand, application of formulas (3.7) and (6.4) to (6.8) yields the relation

$$
\left[\operatorname{Im}\left(b_{k} b_{r}^{-1}\right)\right] Q_{0}^{*} \varphi+\left(P_{k r} \varphi\right)^{+}=\left[\operatorname{Re}\left(b_{k} b_{r}^{-1}\right)\right] \varphi+\operatorname{Re}\left[b_{k} S\left(b_{r}^{-1} \varphi\right] .\right.
$$

Together with the previous equality this implies (6.9). 
Due to (6.5) from (6.8) and Theorems 2.1, 2.2 it immediately follows that the following pairs are the solution $u$ of the Lamé system and its conjugate function $v$ :

$$
\begin{array}{ll}
u=P_{11} \varphi, & v=\left[\operatorname{Im}\left(c b^{-1}\right)\right] Q_{0} \varphi+P_{21} \varphi ; \\
v=P_{22} \varphi, & u=\left[\operatorname{Im}\left(b c^{-1}\right)\right] Q_{0} \varphi+P_{12} \varphi .
\end{array}
$$

Accordingly, it is natural to call the integrals $P_{11} \varphi$ and $P_{22} \varphi$ generalized double layer potentials for the solutions of the Lamé system and their conjugate functions, respectively.

The previous results extend to the spaces of continuous functions as well.

Lemma 6.2. Let $\Gamma \in C^{1, \nu}, 0<\mu<\nu$ and let $X$ denote any of the symbols $C, C^{\mu}, C^{1, \mu}$. Then the operator $P_{k r}$ is bounded $X(\Gamma) \rightarrow X(\bar{D})$, and the operator $P_{k r}^{*}$ is compact in $X(\Gamma)$.

Proof. First consider the operators $P_{k r}$. The boundedness of these operators $C^{\mu}(\Gamma) \rightarrow C^{\mu}(\bar{D})$ follows from equality (6.8) and from the similar property of the operators $Q$ and $I$. For the classes $C^{1, \mu}$, the proof is based on the differentiation formula for the Cauchy type integral $\phi=I \varphi$. Let $\varphi \in C^{1}(\Gamma)$ and $D \varphi$ denote the derivative $\varphi$ in the arc length parameter on $\Gamma$ measured in the positive direction. Then one has

$$
\begin{gathered}
\frac{\partial \phi}{\partial x}(z)=\frac{1}{2 \pi i} \int_{\Gamma}\left[(t-z)_{J}^{-2} e_{J}(t) \varphi(t)|d t|=-\frac{1}{2 \pi i} \int_{\Gamma}\left[D(t-z)_{J}^{-1}\right] \varphi(t)|d t|,\right. \\
\frac{\partial \phi}{\partial y}(z)=\frac{1}{2 \pi i} \int_{\Gamma}\left[J(t-z)_{J}^{-2} e_{J}(t) \varphi(t)|d t|=-\frac{1}{2 \pi i} \int_{\Gamma}\left[D J(t-z)_{J}^{-1}\right] \varphi(t)|d t|,\right.
\end{gathered}
$$

which after a partial integration yields differentiation formulas

$$
\frac{\partial(I \varphi)}{\partial x}=I\left(e_{J}^{-1} D \varphi\right), \quad \frac{\partial(I \varphi)}{\partial y}=I\left(J e_{J}^{-1} D \varphi\right) .
$$

Due to (6.4) we have similar formulas for the operator $Q_{0}$ :

$$
\frac{\partial(Q \varphi)}{\partial x}=-\operatorname{Im}\left[\frac{1}{\pi i} \int_{\Gamma} \frac{(D \varphi(t)|d t|}{t-z}\right], \quad \frac{\partial(Q \varphi)}{\partial y}=-\operatorname{Im}\left[\frac{1}{\pi} \int_{\Gamma} \frac{(D \varphi(t)|d t|}{t-z}\right] .
$$

Applying these formulas to (6.8), we arrive at the boundedness of the operators $P_{k r}: C^{1, \mu}(\Gamma) \rightarrow$ $C^{1, \mu}(\bar{D})$.

The proof of the assertion of the Lemma under consideration for the spaces $C$ is based on the estimate

$$
\sup _{z \in D} \int_{\Gamma} \frac{|\operatorname{Re}[\overline{n(t)}(t-z)]|}{|t-z|^{2}}|d t|<\infty
$$

which is well known for the classical double layer potentials. Since the elements of the homogeneous matrix function $H$ of order 0 do not exceed some constant in the absolute value, we have

$$
\sup _{z \in D} \int_{\Gamma} \frac{|\operatorname{Re}[\overline{n(t)}(t-z)]|}{|t-z|^{2}}\left|H_{k r}(t-z)\right||d t|<\infty,
$$

where under $|H(t-z)|$ we understand some norm in $\mathbb{R}^{2 \times 2}$. Therefore we will establish the assertion under consideration if we show that for $\varphi \in C(\Gamma)$ the function $\left(P_{k r} \varphi\right)(z), z \in D$, has a limit at a fixed boundary point $t_{0} \in \Gamma$. According to (6.8), the operator $P_{k r}$ takes constant vector functions into constant ones. Therefore we can consider without loss of generality that $\varphi\left(t_{0}\right)=0$. If $\Gamma_{0} \subseteq \Gamma$ is a neighborhood of the point $t_{0}$, then, evidently,

$$
\int_{\Gamma \backslash \Gamma_{0}} \frac{\operatorname{Re}[\overline{n(t)}(t-z)]}{|t-z|^{2}} H(t-z) \varphi(t)|d t| \rightarrow \int_{\Gamma \backslash \Gamma_{0}} \frac{\operatorname{Re}\left[\overline{n(t)}\left(t-t_{0}\right)\right]}{\left|t-t_{0}\right|^{2}} H\left(t-t_{0}\right) \varphi(t)|d t|
$$


as $t \rightarrow t_{0}$. On the other hand, by (6.14) with an appropriate choice of $\Gamma_{0}$ a similar integral over $\Gamma_{0}$ can be made arbitrarily small in absolute value uniformly in $z$.

We turn to the operators $P_{k r}^{*}$. Under the assumption $\Gamma \in C^{1, \nu}$ their compactness in the spaces $C^{\mu}(\Gamma)$ and $C^{\mu}(\Gamma), 0<\mu<\nu$, is established with the help of Lemma 3.1 in a way completely similar to the proof of Lemma 3.2. One must just consider that the homogeneous matrix function $H_{k r}(\xi)$ of order zero is even. As for the last case of the space $C^{1, \mu}$, we preliminarily deduce the differentiation formula

$$
D P_{k r}^{*} \varphi=\widetilde{P}_{k r}^{*} D \varphi, \quad \varphi \in C^{1, \mu}(\Gamma)
$$

where the operator $\widetilde{P}_{k r}^{*}$ is obtained by a change of $n(t)$ with $n\left(t_{0}\right)$ in the integral on the left-hand side of $(6.7)$.

The proof is based on the use of differentiation formulas (6.12) and (6.13). Let the operator $\widetilde{Q}^{*}$ be obtained from $Q^{*}$ in a similar way by a change of $n(t)$ with $n\left(t_{0}\right)$ in the integral. Also, put $\widetilde{S}=e_{J} S e_{J}^{-1}$ or, in the explicit form,

$$
(\widetilde{S} \varphi)\left(t_{0}\right)=\frac{1}{\pi i} \int_{\Gamma} e_{J}\left(t_{0}\right)\left(t-t_{0}\right)_{J}^{-1} \varphi(t)|d t|, \quad t_{0} \in \Gamma .
$$

Then, as in the proof of Lemma 6.1, we see that an equality similar to (6.10) holds for the operators under consideration as well:

$$
\left[\operatorname{Im}\left(b_{k} b_{r}^{-1}\right)\right] \widetilde{Q}_{0}^{*} \varphi+\widetilde{P}_{k r}^{*} \varphi=\operatorname{Re}\left[b_{k} \widetilde{S}\left(b_{r}^{-1} \varphi\right)\right] .
$$

Further fix a point $t_{0} \in \Gamma$ and substitute the partial derivatives of (6.12) into the expression

$$
e_{1}\left(t_{0}\right) \frac{\partial(I \varphi)}{\partial x}(z)+e_{2}\left(t_{0}\right) \frac{\partial(I \varphi)}{\partial y}(z) .
$$

Then in the limit as $z \rightarrow t_{0}$ due to the Sohocki-Plemelj Formulas $(3.7)$ we get $2 D(I \varphi)^{+}=D \varphi+\widetilde{S} D \varphi$. On the other hand, differentiation of the Sohocki-Plemelj Formula yields a similar equality $2 D(I \varphi)^{+}=$ $D \varphi+D S \varphi$. Comparing it with the previous one, we obtain a differentiation formula $D S=\widetilde{S} D$ for the singular operator $S$. In a completely similar way, using (6.13), we obtain the equality $D Q=\widetilde{Q}_{0} D$ for the operator $Q$. Acting on equality (6.10) by the operator $D$ and applying these formulas, we obtain

$$
\left[\operatorname{Im}\left(b_{k} b_{r}^{-1}\right)\right] \widetilde{Q} D \varphi+D P_{k r}^{*} \varphi=\operatorname{Re}\left[b_{k} \widetilde{S}\left(b_{r}^{-1} \varphi\right)\right] .
$$

Together with (6.16) this implies (6.15).

As above, we see that the operator $\widetilde{P}_{k r}^{*}$ is compact in the space $C^{\mu}(\Gamma)$. Due to $(6.15)$ this implies the compactness of the operator $P_{k r}^{*}$ in $C^{1, \mu}(\Gamma)$, which completes the proof of the Lemma.

If the conjugate function is represented by one of the two ways (6.11), by formulas (2.16) one can determine the elements of the stress tensor $\sigma$. Therefore the differentiation formulas for the function $v$ play an important role in representations (6.11). They immediately follow from relation (6.8) of Lemma 6.1 and differentiation formulas (6.12) of the Cauchy type integral.

Lemma 6.3. Let $\varphi \in C^{1}(\Gamma)$ and $D \varphi$ denote the derivative of $\varphi$ in the tangential direction $e=$ in on Г. Then

$$
\begin{aligned}
& \frac{\partial v}{\partial x}(z)=\frac{1}{\pi} \int_{\Gamma} \operatorname{Im}\left[c(t-z)_{J}^{-1} c^{-1}\right](D \varphi)(t)|d t|, \\
& \frac{\partial v}{\partial y}(z)=\frac{1}{\pi} \int_{\Gamma} \operatorname{Im}\left[c J(t-z)_{J}^{-1} c^{-1}\right](D \varphi)(t)|d t|,
\end{aligned}
$$

if $v=P_{22} \varphi$, and

$$
\frac{\partial v}{\partial x}(z)=\frac{1}{\pi} \int_{\Gamma} \operatorname{Im}\left[c(t-z)_{J}^{-1} b^{-1}\right](D \varphi)(t)|d t|,
$$




$$
\frac{\partial v}{\partial y}(z)=\frac{1}{\pi} \int_{\Gamma} \operatorname{Im}\left[c J(t-z)_{J}^{-1} b^{-1}\right](D \varphi)(t)|d t|,
$$

if the function $v$ is the conjugate of $u=P_{11} \varphi$.

\section{Integral Representations of Double Layer Potentials}

Consider the representation of the solutions of the Lamé system and their conjugate functions by generalized double layer potentials. Preliminarily we describe the kernel ker $P_{11}=\left\{\varphi \in L^{p}(\Gamma) \mid P_{11} \varphi=\right.$ 0 \} of the operator $P_{11}$. By Theorem 3.1 the analogous kernel of the operator $I$ consists of complex functions that are constant on simple contours making $\Gamma$ (and vanish on the external contour in the case $\sigma(D)=1$ of a bounded domain). Therefore due to (6.8) analogous real vector functions belong to the kernel ker $P_{11}$. In fact, they completely describe this kernel.

Lemma 7.1. Let the domain $D$ be bounded by the contour $\Gamma \in C^{1, \nu}$ consisting of connected components $\Gamma_{1}, \ldots, \Gamma_{m}$, and in the case of a bounded domain let the contour $\Gamma_{m}$ encompass all the other components.

Then the kernel of the operator $P_{11}$ consists of functions that are constant on the connected components $\Gamma$ and vanish on $\Gamma_{m}$ in the case of a bounded domain. In particular, $\operatorname{dim}\left(\operatorname{ker} P_{11}\right)=2[m-\sigma(D)]$.

Proof. Write equality (6.8) for the operator under consideration:

$$
P_{11} \varphi=2 \operatorname{Re}\left[b I\left(b^{-1} \varphi\right)\right] .
$$

Assuming $P_{11} \varphi=0$, consider the Cauchy type integral $\phi=I\left(b^{-1} \varphi\right)$ in the domain $D$ and the analogous integral in the complement $\widetilde{D}=\mathbb{C} \backslash \bar{D}$, which will be denoted by $\psi=\widetilde{I}\left(b^{-1} \varphi\right)$. For the boundary values of these functions by (3.7) one can write:

$$
\phi^{+}-\psi^{-}=b^{-1} \varphi
$$

where we have taken into account the fact that the contour $\Gamma$ is negatively oriented with respect to $\widetilde{D}$.

According to (7.1) the assumption $P_{11} \varphi=0$ means that $\operatorname{Re} b \phi=0$. Therefore the function $\phi$ is constant in the domain $D$; more precisely,

$$
b \phi=i \xi, \xi \in \mathbb{R}^{2} .
$$

By $(7.2)$, since $\varphi$ is real, hence we get

$$
\operatorname{Im} b \psi^{-}=\xi, \quad \varphi=-\operatorname{Re} b \psi^{-} .
$$

Consequently, the function $u_{0}=(\operatorname{Im} b \psi)-\xi$ is a solution of the homogeneous Dirichlet problem for the Lamé system in the connected components of the open set $\widetilde{D}$, and by Theorem 5.1 it is identical zero. Therefore the function $\psi$ is constant in the connected components of $\widetilde{D}$. If the domain $D$ is bounded, then the domain $\widetilde{D}_{m}$ is unbounded, and since $\psi(\infty)=0$, the function $\psi=0$ in $\widetilde{D}_{m}$. Together with the second equality (7.3) this implies that the function $\varphi$ is constant on the contours $\Gamma_{j}$ and in the case $\sigma(D)=1$ it vanishes on $\Gamma_{m}$.

The next main theorem similarly to 3.1 solves the problem of representation of the solutions of the Lamé system by generalized double layer potentials. According to (7.1), the function $u=P_{11} \varphi$ can be written in the form $u=\operatorname{Re} b \phi$, where the $J$-analytic function $\phi=I\left(b^{-1} \varphi\right)$ is single-valued in the domain $D$. Thus the conjugate function of $u=P_{11} \varphi$ is single-valued in the domain $D$. In addition, in the case $\sigma(D)=0$ the function $P_{11} \varphi$ vanishes at infinity. Therefore in notation (4.12), (4.13) functions 
of the form

$$
u=\left\{\begin{array}{ll}
\sum_{j=1}^{m-1} \operatorname{Re} b L_{j} \eta_{j}, & \sigma(D)=1, \\
\xi+\sum_{j=1}^{m-1} \operatorname{Re} b L_{j} \eta_{j}, & \sigma(D)=0,
\end{array} \quad \operatorname{Re} b \eta_{j}=0, \xi \in \mathbb{R}^{2},\right.
$$

cannot be represented by the potential $P_{11} \varphi$. Note that these functions form a space of dimension $2[m-\sigma(D)]$. In fact, the complement to this space is the image of the operator $P_{11}$.

Theorem 7.1. Let the domain $D$ be bounded by the contour $\Gamma \in C^{1, \nu}$ consisting of connected components $\Gamma_{1}, \ldots, \Gamma_{m}$, and in the case of a bounded domain let the contour $\Gamma_{m}$ encompass all the other components. Then any solution $u \in h^{p}(D), 1<p<\infty$, of the Lamé system is representable in the form

$$
u=P_{11} \varphi+u_{0}
$$

with some $\varphi \in L^{p}(\Gamma)$ and the function $u_{0}$ of form (7.4), and the last function is uniquely determined by $u$.

If $u \in X(\bar{D})$, where $X$ denotes any of the symbols $C, C^{\mu}, C^{1, \mu}, \mu<\nu$, then also $\varphi \in X(\Gamma)$.

Proof. The proof of the first assertion is almost obvious. By Lemma 6.1 the composition of $P_{11}$ with the operator of the Dirichlet problem (2.1) is a Fredholm operator $1+P_{11}^{*}$ with index zero, therefore due to Theorem 4.2 and well-known properties of Fredholm operators [14] the operator $P_{11}$ is also Fredholm and its index equals 1 . In particular, the image im $P_{11}$ is closed and its codimension coincides with the dimension $2\left[m-\sigma(D)\right.$ of the kernel ker $P_{11}$. Since the space of functions (7.4) has the same dimension and does not intersect the image $\Im P_{11}$, this implies the validity of the first part of the Theorem.

The second assertion is a corollary of Lemma 4.1. In fact, if $u \in X(\bar{D})$, then according to (7.5) the function $\varphi \in L^{p}(\Gamma)$ is a solution of equation $\varphi+P_{11}^{*} \varphi=f_{1}$ with the right-hand side $f_{1}=u^{+}-u_{0}^{+} \in$ $X(\Gamma)$. By Lemma 6.2, the operator $P_{11}^{*}$ is compact both in $L^{p}(\Gamma)$ and in $X(\Gamma)$, so that the identity operator is a regularizer of $1+P_{11}^{*}$. Therefore due to Lemma 4.1 the function $\varphi \in X(\Gamma)$.

Theorem 7.1 allows one to reduce the Dirichlet problem in the space $h^{p}(D)$ to an equivalent Fredholm integral equation in $L^{p}(\Gamma)$. Let $k=2\left[m-\sigma(D)\right.$ and $g_{1}, \ldots, g_{k}$ be the base of the space ker $P_{11}$. Let also $u_{1}, \ldots, u_{k}$ be the base of the space of functions of form (7.4). Then by Theorem 7.1 the operator

$$
\widetilde{P} \varphi=P_{11} \varphi+\sum_{1}^{k}\left(\varphi, g_{j}\right) u_{j}, \quad(\varphi, g)=\int_{\Gamma} \varphi(t) g(t)|d t|,
$$

is invertible $L^{p}(\Gamma) \rightarrow h^{p}(D)$ and the Dirichlet problem $u^{+}=f$ reduces to the equivalent Fredholm equation of the second kind

$$
\varphi+P_{11}^{*} \varphi+\sum_{1}^{k}\left(\varphi, g_{j}\right) u_{j}^{+}=f .
$$

If $\varphi$ is a solution of this equation, then the first pair in (6.11) defines the solution $u$ of the Dirichlet problem and the respective conjugate function.

As we see from the proof of Theorems 3.1 and 5.1, the solvability of the Dirichlet problem for the Lamé system reduces to that of a singular integral equation on $\Gamma$, which does not allow to consider this problem in the class $C(\bar{D})$ in the framework of this approach. It is known [11] that the construction of classical double layer potentials is based on the fundamental matrix of solutions for the original elliptic system. For the Lamé system versions of matrices of this type were suggested in [10], but the double layer potentials constructed with their help also reduce the main boundary value problems for the Lamé system to singular integral equations on the boundary. An advantage of the generalized double layer potentials under consideration $u=P_{11} \varphi$ is the possibility they give to reduce the problem 
to the Fredholm Eq. (7.6) which is free from the aforementioned drawback. Note that these potentials are related to the version $\operatorname{Re}\left[(2 \pi i)^{-1} b \ln z_{J}\right]$ of the fundamental matrix of the Lamé system.

We turn our attention to he operator $P_{22}$ and, similarly to the above, first describe its whole kernel.

Lemma 7.2. Under the hypotheses of Lemma 7.1 the kernel ker $P_{22}$ of the operator $P_{22}$ consists of functions $\varphi$ such that in notation (2.30) one has

$$
\begin{gathered}
\left.\varphi\right|_{\Gamma_{j}}=\xi_{j}+\left.\lambda_{j}\left(x \xi^{0}+y \xi^{1}\right)\right|_{\Gamma_{j}}, \quad 1 \leq j \leq m-\sigma(D), \\
\left.\varphi\right|_{\Gamma_{m}}=\lambda_{m} \operatorname{Im}\left(\left.c \psi_{0}\right|_{\Gamma_{m}}\right), \quad \sigma(D)=1,
\end{gathered}
$$

where $\xi_{j} \in \mathbb{R}^{2}, \lambda_{j} \in \mathbb{R}$ and the function $\psi_{0}$ is defined as in Lemma 5.1 with respect to $\widetilde{D}_{m}$.

In particular, ker $P_{22} \supseteq \operatorname{ker} P_{11}$ and dim ker $P_{22}=3 m-2 \sigma(D)$.

Proof. It is similar to that of Lemma 7.1. Let $P_{22} \varphi=0$, so that for the integral of the Cauchy type $\phi=I\left(c^{-1} \varphi\right)$ and $\psi=\widetilde{I}\left(c^{-1} \varphi\right)$ in $D$ and $\widetilde{D}$, respectively, we have the relations

$$
\operatorname{Re} c \phi=0
$$

and $\phi^{+}-\psi^{-}=c^{-1} \varphi$. The last complex equality due to (7.9) is equivalent to two real ones:

$$
\begin{gathered}
\operatorname{Im} c \psi^{-}=\operatorname{Im} c \phi^{+}, \\
\varphi=-\operatorname{Re} c \psi^{-} .
\end{gathered}
$$

The converse is also true: if some functions $\phi$ and $\psi$ in the class $H^{p}$ satisfy (7.9) and (7.10), then $\varphi=-\operatorname{Re} c \psi^{-} \in \operatorname{ker} P_{22}$. In fact, then we have $\phi^{+}-\psi^{-}=c^{-1} \varphi$ and the Cauchy formula (3.3) applied to $\phi$ in the domain $D$ and to $\psi$ in the connected components of $\widetilde{D}$ yields equalities

$$
\phi=I\left(c^{-1} \varphi\right), \quad \psi=\widetilde{I}\left(c^{-1} \varphi\right),
$$

the first of which together with (7.9) means $P_{22} \varphi=0$.

The further arguments will be given separately for the two cases of the bounded and unbounded domains.

1) Let the domain $D$ be unbounded, Then by Theorem 2.2 from (7.9) follows $\phi=0$ and (7.10) becomes the equality $\operatorname{Im} c \psi^{-}=0$. Then by Theorem 5.2 in each component $\widetilde{D}_{j}$ of the open set $\widetilde{D}$ the function $\operatorname{Im} c \psi$ identically equals zero. By Theorem 2.2 we conclude that in the domain $\widetilde{D}_{j}$ the function $i \psi(z)$ is a polynomial of form (2.29), i.e., in the notation of Lemma 2.3 one has

$$
\psi(z)=i\left(\eta_{j}+\lambda_{j} z_{J} e\right), \quad z \in \widetilde{D}_{j},
$$

with some $\eta_{j} \in \mathbb{C}^{2}$ and $\lambda_{j} \in \mathbb{R}$. It remains to note that $\varphi=-\operatorname{Re} c \psi^{-}$for functions of form (7.11) are described on $\Gamma_{j}$ by equality (7.7).

2) Let the domain $D$ be bounded. Then Eq. (7.9) defines the polynomials $\phi=p$ of form (7.12):

$$
p(z)=\eta+\lambda z_{J} e, \quad z \in D .
$$

In the case $j \leq m-1$ problem (7.10) for the function $\psi$ in the bounded domain $\widetilde{D}_{j}$ can be rewritten in the form $\operatorname{Re} c i(\psi-p)^{-}=0$ so that $\psi-p$ is defined by the right-hand side of (7.12). Since $\operatorname{Re} c p=0$, for the function $\varphi=-\operatorname{Re} c \psi^{-}$we have expression (7.7).

As for the unbounded domain $\widetilde{D}_{m}$, equation (7.10) with respect to $\phi_{0}=-i \psi$ in this domain can be written in the form

$$
\operatorname{Re} c \phi_{0}^{-}=\left.\operatorname{Re} c(-i p)\right|_{\Gamma_{m}} .
$$

By Lemma 2.3 we can apply Lemma 5.1 to the problem under consideration, which yields the existence of a unique function $\psi=i \phi_{0}$ satisfying this boundary condition. Accordingly, $\varphi=-\operatorname{Re} c \psi^{-}$on $\Gamma_{m}$ is defined by formula (7.8). 
Note that under the assumption $\Gamma \in C^{1, \nu}$ function (7.8) belongs to the class $C^{1, \mu}(\Gamma)$ for any $\mu<\nu$. The class of functions of this type is briefly denoted as $C^{1, \nu-0}(\Gamma)$.

Consider representation of conjugate functions by potentials $P_{22} \varphi$. As in the case of solutions (7.4) of the Lamé system we see that the functions of the form

$$
v=\left\{\begin{array}{ll}
\sum_{j=1}^{m-1} \operatorname{Re} c L_{j} \eta_{j}, & \sigma(D)=1, \\
\xi+\sum_{j=1}^{m-1} \operatorname{Re} c L_{j} \eta_{j}, & \sigma(D)=0,
\end{array} \quad \operatorname{Re} c \eta_{j}=0, \xi \in \mathbb{R}^{2},\right.
$$

cannot be represented by the potential $P_{22} \varphi$.

Theorem 7.2. Under the hypotheses of Theorem 7.1 there exists a finite dimensional space $V \subseteq$ $C^{1, \nu-0}(\bar{D})$ of dimension $3 m-2 \sigma(D)-1$ containing functions of the form (7.13) such that any function $v \in h^{p}(D)$ conjugate to some (generally multivalued) solution of the Lamé system is representable in the form

$$
v=P_{22} \varphi+v_{0}
$$

with some $\varphi \in L^{p}(\Gamma)$ and $v_{0} \in V$ so that $v_{0}$ in this representation is uniquely determined by $v$.

If $v \in X(\bar{D})$, where $X$ denotes any symbol $C, C^{\mu}, C^{1, \mu}, \mu<\nu$, then also $\varphi \in X(\Gamma)$.

Proof. It is completely analogous to that of Theorem 7.1. Since the composition of $P_{22}$ with the operator of the Dirichlet problem (5.12) is a Fredholm operator $1+P_{22}^{*}$ of index zero, due to Theorem 5.2 we conclude that the operator $P_{22}$ is Fredholm and its index equals $\sigma(D)$. Therefore its image im $P_{22}$ is closed and has the codimension equal to $k=\operatorname{dim}\left(\operatorname{ker} P_{22}\right)-\sigma(D)$. Due to Lemma 6.2 similar arguments hold for the operator $P_{22}$ acting from $X(\bar{D})$ into $X(\Gamma)$ as well. In particular, in $C^{1, \mu}(\bar{D})$ there exists a subspace $V$ of dimension $k$ containing functions (7.13) such that decomposition (7.14) holds for functions $v \in(\bar{D})$. By dimension arguments this decomposition also takes place in $h^{p}(D)$.

The second assertion of the Theorem is proved with the help of Lemma 4.1 in a way completely similar to the preceding one.

As above, Theorem 7.2 allows one to reduce the Dirichlet problem for conjugate functions to an equivalent system of Fredholm boundary equations. Some changes are required only in the case $\sigma(D)=1$ of a bounded domain $D$ we consider here. Put $k=\operatorname{dim}\left(k e r P_{22}\right)-1$ and introduce bases $g_{1}, \ldots, g_{k+1}$ and $v_{1}, \ldots, v_{k}$, respectively, in the spaces $k e r P_{22}$ and $V$. Consider the operator

$$
N \varphi=\varphi+P_{22}^{*} \varphi+\sum_{1}^{k}\left(g_{j}, \varphi\right) v_{j}^{+}+\left(g_{k+1}, \varphi\right) n,
$$

where, as we recall, the function $n=\left(n_{1}, n_{2}\right)$ denotes the exterior unit normal. Evidently, the functions $\varphi+P_{22}^{*} \varphi=\left(P_{22} \varphi\right)^{+}$and $v_{j}^{+}$satisfy the condition of orthogonality to $n$. Therefore, if $N \varphi=0$, then $\left(g_{k+1}, \varphi\right)=0$; due to Theorem 7.2 we also have relations $N_{22} \varphi=0$ and $\left(g_{j}, \varphi\right)=0, \quad 1 \leq j \leq k$, whence $\varphi=0$. Thus the operator $N$ is invertible, and the function $N \varphi$ is orthogonal to $n$ if and only if $\left(g_{k+1}, \varphi\right)=0$. Therefore under the assumption $(f, n)=0$ the solution $\varphi$ of equation $N \varphi=f$ yields the solution of the Dirichlet problem by the formula

$$
v=P_{22} \varphi+\sum_{1}^{k}\left(g_{j}, \varphi\right) v_{j}
$$




\section{Structure of Matrices $H_{k r}(\xi)$}

Using notation (1.7), introduce the quadratic forms

$$
\begin{gathered}
\omega(\xi)=\left(\xi_{1}+\nu_{1} \xi_{2}\right)\left(\xi_{1}+\nu_{2} \xi_{2}\right)=\xi_{1}^{2}+s \xi_{1} \xi_{2}+t \xi_{2}^{2}, \\
2 \omega_{1}(\xi)=s \xi_{1}^{2}+2 t \xi_{1} \xi_{2}+\bar{s} t \xi_{2}^{2}, \\
\Omega(\xi)=\left(\begin{array}{cc}
{\left[(t-1) \xi_{1} \xi_{2}+s \xi_{1}^{2}\right]} & t|\xi|^{2} \\
-|\xi|^{2} & (t-1) \xi_{1} \xi_{2}-s \xi_{2}^{2}
\end{array}\right) .
\end{gathered}
$$

Evidently, it suffices to describe the matrix function

$$
G_{k r}(\xi)=|\xi|^{-2}|\omega(\xi)|^{2} H_{k r}(\xi),
$$

which is homogeneous of order 2 .

Theorem 8.1. The matrices $G_{k r}$ are defined by the formulas

$$
G_{11}(\xi)=\operatorname{Im}\left[\omega_{1}(\xi)+\bar{\omega}(\xi)\left(b \Delta b^{-1}\right)\right],
$$

where in parallelism with the two cases $(i)$ and $(i i)$ we put

$$
\text { (i) } \Delta=\frac{1}{2}\left(\begin{array}{cc}
\nu_{1}-\nu_{2} & 0 \\
0 & \nu_{2}-\nu_{1}
\end{array}\right), \quad \text { (ii) } \Delta=\left(\begin{array}{ll}
0 & 1 \\
0 & 0
\end{array}\right) \text {, }
$$

and

$$
\begin{gathered}
G_{22}(\xi)=\operatorname{Im}\left(\begin{array}{cc}
s \xi_{1}^{2}+t \xi_{1} \xi_{2} & t \xi_{1}^{2}+\bar{s} t \xi_{1} \xi_{2} \\
s \xi_{1} \xi_{2}+t \xi_{2}^{2} & t \xi_{1} \xi_{2}+\bar{s} t \xi_{2}^{2}
\end{array}\right), \\
G_{21}(\xi)=|\xi|^{-2} \operatorname{Re}[\bar{\omega}(\xi) \Omega(\xi)] \operatorname{Im}\left(c b^{-1}\right)+G_{22}(\xi) \operatorname{Re}\left(c b^{-1}\right), \\
G_{12}(\xi)=|\xi|^{-2} \operatorname{Im}\left(b c^{-1}\right) \operatorname{Re}[\bar{\omega}(\xi) \Omega(\xi)]+\operatorname{Re}\left(b c^{-1}\right) G_{22}(\xi) .
\end{gathered}
$$

Proof. We prove the first equality (8.3) for the cases $(i)$ and (ii) separately.

(i). We put

then we have

$$
h(\xi, \nu)=\frac{-\xi_{2}+\nu \xi_{1}}{\xi_{1}+\nu \xi_{2}},
$$

$$
\left(-\xi_{2}+\xi_{1} J\right)\left(\xi_{1}+\xi_{2} J\right)^{-1}=\operatorname{diag}\left[h\left(\xi, \nu_{1}\right), h\left(\xi, \nu_{2}\right)\right] .
$$

By definition of the matrix $\Delta$ this implies

$$
\left(-\xi_{2}+\xi_{1} J\right)\left(\xi_{1}+\xi_{2} J\right)^{-1}=\frac{h\left(\xi, \nu_{1}\right)+h\left(\xi, \nu_{2}\right)}{2}+\frac{h\left(\xi, \nu_{1}\right)-h\left(\xi, \nu_{2}\right)}{\nu_{1}-\nu_{2}} \Delta .
$$

It is easy to see that

$$
\frac{h\left(\xi, \nu_{1}\right)+h\left(\xi, \nu_{2}\right)}{2}=\frac{\omega_{0}(\xi)}{2 \omega(\xi)}, \quad \frac{h\left(\xi, \nu_{1}\right)-h\left(\xi, \nu_{2}\right)}{\nu_{1}-\nu_{2}}=\frac{|\xi|^{2}}{\omega(\xi)},
$$

where $\omega_{0}(\xi)=\left(-\xi_{2}+\nu_{1} \xi_{1}\right)\left(\xi_{1}+\nu_{2} \xi_{2}\right)+\left(-\xi_{2}+\nu_{2} \xi_{1}\right)\left(\xi_{1}+\nu_{1} \xi_{2}\right)$.

Therefore

$$
2|\xi|^{-2}|\omega(\xi)|^{2} b\left[\left(-\xi_{2}+\xi_{1} J\right)\left(\xi_{1}+\xi_{2} J\right)^{-1}\right] b^{-1}=|\xi|^{-2} \omega_{0}(\xi) \overline{\omega(\xi)}+2 \overline{\omega(\xi)}\left(b \Delta b^{-1}\right) .
$$

It is easy to check that

$$
\operatorname{Im} h(\xi, \nu)=\frac{|\xi|^{2} \operatorname{Im} \nu}{\left|\xi_{1}+\nu \xi_{2}\right|^{2}}
$$

so that by definition (8.1)

$$
\operatorname{Im}\left[h\left(\xi, \nu_{1}\right)+h\left(\xi, \nu_{2}\right)\right]=\frac{|\xi|^{2} \omega_{1}(\xi)}{|\omega(\xi)|^{2}}
$$


Together with the first equality (8.7) this leads to the relation

$$
\operatorname{Im}\left[\left(\omega_{0}(\xi) \overline{\omega(\xi)}\right]=|\xi|^{2} \operatorname{Im}\left[\omega_{1}(\xi)\right]\right.
$$

for quadratic forms. Therefore according to definitions (6.5), (8.2) the imaginary part of equality (8.8) coincides with (8.3).

(ii). By definition of the matrix $\Delta$ in this case

$$
\left(-\xi_{2}+\xi_{1} J\right)\left(\xi_{1}+\xi_{2} J\right)^{-1}=h(\xi, \nu)\left(1+\frac{\xi_{1}}{-\xi_{2}+\nu \xi_{1}} \Delta\right)\left(1+\frac{\xi_{2}}{\xi_{1}+\nu \xi_{2}} \Delta\right)^{-1},
$$

so that

$$
\left(-\xi_{2}+\xi_{1} J\right)\left(\xi_{1}+\xi_{2} J\right)^{-1}=h(\xi, \nu)+\frac{|\xi|^{2}}{\omega(\xi)^{2}} \Delta .
$$

Putting $\nu_{k}=\nu$ in the first relation (8.7), we obtain the equality

$$
h(\xi, \nu)=\frac{\omega^{1}(\xi)}{2 \omega(\xi)},
$$

which similarly to the previous case $(i)$ leads to $(8.3)$.

We prove the remaining formulas of the theorem. Equality (8.8) holds for the matrix $c$ as well. Since the matrix $d$, which appears in (2.25), commutes with $\Delta$, the matrix $c \Delta c^{-1}$ in the right-hand side of this equality can be replaced by the matrix $c_{0}$. A direct check shows that in both cases $(i)$ and (ii) the product

$$
c_{0} \Delta c_{0}^{-1}=\frac{1}{2}\left(\begin{array}{cc}
s & 2 t \\
-2 & -s
\end{array}\right)
$$

Thus we have

$$
2|\omega(\xi)|^{2} c\left[\left(-\xi_{2}+\xi_{1} J\right)\left(\xi_{1}+\xi_{2} J\right)^{-1}\right] c^{-1}=\omega_{0}(\xi) \overline{\omega(\xi)}+2 \overline{\omega(\xi)}\left(c_{0} \Delta c_{0}^{-1}\right) .
$$

By definition of the quadratic form $\omega_{0}$ in (8.7) we see that it can be written in notation (1.7) as $\omega_{0}(\xi)=2(t-1) \xi_{1} \xi_{2}+s\left(\xi_{1}^{2}-\xi_{2}^{2}\right)$. A direct check shows that the expression $\omega_{0}(\xi)+2|\xi|^{2} c_{0} \Delta c_{0}^{-1}$ coincides with the matrix $2 \Omega(\xi)$ in $(8.1)$, so that

$$
|\omega(\xi)|^{2} c\left[\left(-\xi_{2}+\xi_{1} J\right)\left(\xi_{1}+\xi_{2} J\right)^{-1}\right] c^{-1}=\Omega(\xi) \overline{\omega(\xi)} .
$$

Due to $(6.5),(8.2)$ we get

$$
G_{22}(\xi)=|\xi|^{-2} \operatorname{Im}[\Omega(\xi) \overline{\omega(\xi)}],
$$

which immediately results in (8.4). Exactly in the same way, by (8.10) equality

$$
G_{21}(\xi)=|\xi|^{-2}|\omega(\xi)|^{2} \operatorname{Im}\left[c\left(-\xi_{2}+\xi_{1} J\right)\left(\xi_{1}+\xi_{2} J\right)^{-1} c^{-1}\left(c b^{-1}\right)\right]
$$

can be rewritten in the form

$$
G_{21}(\xi)=|\xi|^{-2} \operatorname{Im}\left[\Omega(\xi) \overline{\omega(\xi)}\left(c b^{-1}\right)\right],
$$

whence due to (8.11) the first formula (8.5) follows. The second formula is proved similarly.

Substituting into (8.3)-(8.5) the respective expressions of Lemma 2.2 for the matrices $b$ and $c$, we can calculate the elements of the matrices $G_{k r}$. This can be done most simply with the help of the following bilinear forms of polynomials introduced for each of the two cases of roots $\nu_{j}$ of the characteristic equation of the Lamé system:

$$
\begin{array}{ll}
\text { (i) }[f, g]=\frac{f\left(\nu_{1}\right) g\left(\nu_{2}\right)-f\left(\nu_{2}\right) g\left(\nu_{1}\right)}{\nu_{1}-\nu_{2}}, & (\text { ii })[f, g]=f^{\prime}(\nu) g(\nu)-f(\nu) g^{\prime}(\nu), \\
\text { (i) }\{f, g\}=\frac{f\left(\nu_{1}\right) g\left(\nu_{2}\right)+f\left(\nu_{2}\right) g\left(\nu_{1}\right)}{2}, & \text { (ii) }\{f, g\}=f(\nu) g(\nu) .
\end{array}
$$

In our notation Lemma 2.2 leads to the following expressions for the matrices under consideration. 
Lemma 8.1. If $\alpha \in \mathcal{A}_{1}$, then one has

$$
\begin{aligned}
b \Delta b^{-1} & =\frac{1}{\left[p_{3}, p_{2}\right]}\left(\begin{array}{cc}
-\left\{p_{2}, p_{3}\right\} & -\left\{p_{2}, p_{2}\right\} \\
\left\{p_{3}, p_{3}\right\} & \left\{p_{2}, p_{3}\right\}
\end{array}\right), \\
c b^{-1} & =\frac{1}{\left[p_{3}, p_{2}\right]}\left(\begin{array}{cc}
-\left[p_{3}, q_{3}\right] & -\left[p_{2}, q_{3}\right] \\
{\left[p_{3}, q_{2}\right]} & {\left[p_{2}, q_{2}\right]}
\end{array}\right), \\
b c^{-1} & =\frac{1}{\left[q_{3}, q_{2}\right]}\left(\begin{array}{cc}
-\left[p_{2}, q_{2}\right] & -\left[p_{2}, q_{3}\right] \\
{\left[p_{3}, q_{2}\right]} & {\left[p_{3}, q_{3}\right]}
\end{array}\right) .
\end{aligned}
$$

If $\alpha \in \mathcal{A}_{2}$, then one has

$$
\begin{aligned}
b \Delta b^{-1} & =\frac{1}{\left[p_{1}, p_{3}\right]}\left(\begin{array}{cc}
-\left\{p_{1}, p_{3}\right\} & -\left\{p_{3}, p_{3}\right\} \\
\left\{p_{1}, p_{1}\right\} & \left\{p_{1}, p_{3}\right\}
\end{array}\right), \\
c b^{-1} & =\frac{1}{\left[p_{1}, p_{3}\right]}\left(\begin{array}{cc}
{\left[p_{1}, q_{1}\right]} & {\left[p_{3}, q_{1}\right]} \\
-\left[p_{1}, q_{4}\right] & -\left[p_{3}, q_{4}\right]
\end{array}\right), \\
b c^{-1} & =\frac{1}{\left[q_{1}, q_{4}\right]}\left(\begin{array}{cc}
{\left[p_{3}, q_{4}\right]} & {\left[p_{3}, q_{1}\right]} \\
-\left[p_{1}, q_{4}\right] & -\left[p_{1}, q_{1}\right]
\end{array}\right) .
\end{aligned}
$$

Finally, in the case $\alpha \in \mathcal{A}_{0}$ one has

$$
\begin{gathered}
b \Delta b^{-1}=\frac{\sqrt{\alpha_{1} \alpha_{2}}-\alpha_{3}}{\sqrt{\alpha_{2} \alpha_{3}}}\left(\begin{array}{cc}
i & 0 \\
0 & -i
\end{array}\right), \quad c b^{-1}=\alpha_{3}\left(\begin{array}{cc}
-i \sqrt{\alpha_{1} / \alpha_{3}} & -1 \\
1 & -i \sqrt{\alpha_{2} / \alpha_{3}}
\end{array}\right), \\
b c^{-1}=\frac{1}{\sqrt{\alpha_{1} \alpha_{2}}-\alpha_{3}}\left(\begin{array}{cc}
-i \sqrt{\alpha_{2} / \alpha_{3}} & 1 \\
-1 & -i \sqrt{\alpha_{1} / \alpha_{3}}
\end{array}\right) .
\end{gathered}
$$

Proof. In accordance with the two cases of the roots, relate to the pair of polynomials $f_{1}, f_{2}$ the matrix

$$
\text { (i) } W\left(f_{1}, f_{2}\right)=\left(\begin{array}{ll}
f_{1}\left(\nu_{1}\right) & f_{1}\left(\nu_{2}\right) \\
f_{2}\left(\nu_{1}\right) & f_{2}\left(\nu_{2}\right)
\end{array}\right), \quad(i i) W\left(f_{1}, f_{2}\right)=\left(\begin{array}{ll}
f_{1}(\nu) & f_{1}^{\prime}(\nu) \\
f_{2}(\nu) & f_{2}^{\prime}(\nu)
\end{array}\right) .
$$

It is easy to see that for its determinant we have the expression

$$
\text { (i) } \operatorname{det} W\left(f_{1}, f_{2}\right)=\left(\nu_{1}-\nu_{2}\right)\left[f_{1}, f_{2}\right], \quad(i i) \operatorname{det} W\left(f_{1}, f_{2}\right)=-\left[f_{1}, f_{2}\right],
$$

so that the invertibility of this matrix is ensured by the condition $\left[f_{1}, f_{2}\right] \neq 0$.

According to Lemma 2.2, in this notation we can write

$$
\begin{array}{lll}
b=W\left(p_{2},-p_{3}\right), & c=W\left(-q_{3}, q_{2}\right), & \alpha \in \mathcal{A}_{1}, \\
b=W\left(-p_{3}, p_{1}\right), & c=W\left(-q_{1}, q_{4}\right), & \alpha \in \mathcal{A}_{2} .
\end{array}
$$

A simple calculation shows that in both cases $(i)$ and $(i i)$, the same equalities hold:

$$
\begin{gathered}
W\left(f_{1}, f_{2}\right) \Delta\left[W\left(g_{1}, g_{2}\right)\right]^{-1}=\frac{1}{\left[g_{1}, g_{2}\right]}\left(\begin{array}{ll}
\left\{f_{1}, g_{2}\right\} & -\left\{f_{1}, g_{1}\right\} \\
\left\{f_{2}, g_{2}\right\} & -\left\{f_{2}, g_{1}\right\}
\end{array}\right), \\
W\left(f_{1}, f_{2}\right)\left[W\left(g_{1}, g_{2}\right)\right]^{-1}=\frac{1}{\left[g_{1}, g_{2}\right]}\left(\begin{array}{ll}
{\left[f_{1}, g_{2}\right]} & -\left[f_{1}, g_{1}\right] \\
{\left[f_{2}, g_{2}\right]} & -\left[f_{2}, g_{1}\right]
\end{array}\right) .
\end{gathered}
$$

Substituting expressions (8.16) into these formulas, we arrive at the validity of formulas (8.13) and (8.14).

As for (8.15), these equalities are obtain directly from (1.15) and (2.23).

It is important to note that by Lemma 2.1 for $\alpha \in \mathcal{A}_{1} \cap \mathcal{A}_{2}$ the respective matrices in (8.13) and (8.14) coincide. Explicit expressions for the elements of these matrices are obtained by calculation of forms (8.12) for polynomials (1.6) and (2.20). In particular, formulas (8.3)-(8.5) together with (8.13) show that the kernels $P_{k r}(n, \xi)$ depend only on $\alpha \in \mathcal{A}_{1} \cup \mathcal{A}_{2}$ and are continuous functions of variables $\alpha_{j}$. 
Starting from concrete expressions of polynomials (1.6) and (2.20), we can easily calculate the elements of the matrices on the right-hand side of (8.13) and (8.14). Since the form [, ] is skew symmetric and the form $\{$,$\} symmetric, it suffices to know the values of these forms on the basic$ elements $z^{i}, 0 \leq i \leq 3$. These values depend only on the simplest symmetric combinations of (1.7). In particular, the elements of matrices (8.13) can be calculated by the formulas

$$
\begin{aligned}
& {\left[p_{3}, p_{2}\right]=\alpha_{3}\left(\alpha_{3}+\alpha_{4}\right)-2 \alpha_{5} \alpha_{6}+\left(\alpha_{3} \alpha_{5}-\alpha_{2} \alpha_{6}\right) s+\left[2 \alpha_{5}^{2}-\alpha_{2}\left(\alpha_{3}+\alpha_{4}\right)\right] t} \\
& {\left[q_{3}, q_{2}\right]=\beta_{4}^{2}-\beta_{4} \beta_{6} s+\left(\beta_{6}^{2}-2 \beta_{1} \beta_{4}\right) t+\beta_{1} \beta_{4} s^{2}-\beta_{1} \beta_{6} s t+\beta_{1}^{2} t^{2}} \\
& {\left[p_{2}, q_{2}\right]=2 \alpha_{5} \beta_{4}+\alpha_{3} \beta_{6}+\left(\alpha_{2} \beta_{4}-\alpha_{3} \beta_{1}\right) s-\left(\alpha_{2} \beta_{6}+2 \alpha_{5} \beta_{1}\right) t} \\
& {\left[p_{2}, q_{3}\right]=-\alpha_{3} \beta_{4}+\alpha_{3} \beta_{6} s-\alpha_{3} \beta_{1}\left(s^{2}-t\right)+\left(\alpha_{2} \beta_{4}+2 \alpha_{5} \beta_{6}\right) t-2 \alpha_{5} \beta_{1} s t-\alpha_{2} \beta_{1} t^{2},} \\
& {\left[p_{3}, q_{2}\right]=\left(\alpha_{3}+\alpha_{4}\right) \beta_{4}+\alpha_{6} \beta_{6}+\left(\alpha_{5} \beta_{4}-\alpha_{6} \beta_{1}\right) s-\left[\alpha_{5} \beta_{6}+\left(\alpha_{3}+\alpha_{4}\right) \beta_{1}\right] t,} \\
& {\left[p_{3}, q_{3}\right]=-\alpha_{6} \beta_{4}+\alpha_{6} \beta_{6} s-\alpha_{6} \beta_{1}\left(s^{2}-t\right)+\left[\alpha_{5} \beta_{4}+\left(\alpha_{3}+\alpha_{4}\right) \beta_{6}\right] t} \\
& -\left(\alpha_{3}+\alpha_{4}\right) \beta_{1} s t-\alpha_{5} \beta_{1} t^{2} \\
& \left\{p_{2}, p_{2}\right\}=\alpha_{3}^{2}+4 \alpha_{5}^{2} t+\alpha_{2}^{2} t^{2}+2 \alpha_{3} \alpha_{5} s+\alpha_{2} \alpha_{3}\left(s^{2}-2 t\right)+2 \alpha_{2} \alpha_{5} s t \\
& \left\{p_{3}, p_{3}\right\}=\alpha_{6}^{2}+\left(\alpha_{3}+\alpha_{4}\right)^{2} t+\alpha_{5}^{2} t^{2}+\left(\alpha_{3}+\alpha_{4}\right) \alpha_{6} s+\alpha_{5} \alpha_{6}\left(s^{2}-2 t\right) \\
& +\left(\alpha_{3}+\alpha_{4}\right) \alpha_{5} s t \\
& 2\left\{p_{2}, p_{3}\right\}=2 \alpha_{3} \alpha_{6}+\left[2 \alpha_{5} \alpha_{6}+\alpha_{3}\left(\alpha_{3}+\alpha_{4}\right)\right] s+4\left(\alpha_{3}+\alpha_{4}\right) \alpha_{5} t \\
& +\left(\alpha_{2} \alpha_{6}+\alpha_{3} \alpha_{5}\right)\left(s^{2}-2 t\right)+\left[\alpha_{2}\left(\alpha_{3}+\alpha_{4}\right)+2 \alpha_{5}^{2}\right] s t+2 \alpha_{2} \alpha_{5} t^{2} .
\end{aligned}
$$

In the case of an orthotropic medium the above formulas for the matrices $G(\xi)$ are essentially simplified. In this case either $\alpha \in \mathcal{A}_{1} \cap \mathcal{A}_{2}$ and one can use each group of equalities (8.13) and (8.14), or one has $\alpha \in \mathcal{A}_{0}$. Let $\alpha \in \mathcal{A}_{1} \cap \mathcal{A}_{2}$, i.e., $\alpha_{5}=\alpha_{6}=0$ and $\alpha_{3}+\alpha_{4} \neq 0$. Then also $\beta_{5}=\beta_{6}=0$, so that the preceding formulas take the form

$$
\begin{array}{ll}
{\left[p_{3}, p_{2}\right]=\alpha_{3}\left(\alpha_{3}+\alpha_{4}\right)-\alpha_{2}\left(\alpha_{3}+\alpha_{4}\right) t,} & {\left[q_{3}, q_{2}\right]=\beta_{4}^{2}+\beta_{1} \beta_{4}\left(s^{2}-t\right)-\beta_{1} \beta_{4} t+\beta_{1}^{2} t^{2},} \\
{\left[p_{2}, q_{2}\right]=\left(\alpha_{2} \beta_{4}-\alpha_{3} \beta_{1}\right) s,} & \\
{\left[p_{3}, q_{3}\right]=-\left(\alpha_{3}+\alpha_{4}\right) \beta_{1} s t,} & 2\left\{p_{2}, p_{3}\right\}=\alpha_{3}\left(\alpha_{3}+\alpha_{4}\right) s+\alpha_{2}\left(\alpha_{3}+\alpha_{4}\right) s t, \\
{\left[p_{2}, q_{3}\right]=-\alpha_{3} \beta_{4}-\alpha_{3} \beta_{1}\left(s^{2}-t\right)} & \left\{p_{2}, p_{2}\right\}=\alpha_{3}^{2}+\alpha_{2}^{2} t^{2}+\alpha_{2} \alpha_{3}\left(s^{2}-2 t\right), \\
+\alpha_{2} \beta_{4} t-\alpha_{2} \beta_{1} t^{2}, & \left\{p_{3}, p_{3}\right\}=\left(\alpha_{3}+\alpha_{4}\right)^{2} t . \\
{\left[p_{3}, q_{2}\right]=\left(\alpha_{3}+\alpha_{4}\right) \beta_{4}-\left(\alpha_{3}+\alpha_{4}\right) \beta_{1} t,} &
\end{array}
$$

Since $\beta_{1}=\alpha_{2} \alpha_{3}, \beta_{4}=-\alpha_{3} \alpha_{4}$, due to (1.22), (1.25) after elementary transformations we get

$$
\begin{aligned}
& {\left[p_{3}, p_{2}\right]=\left(\alpha_{3}+\alpha_{4}\right)\left(\alpha_{3}+\sqrt{\alpha_{1} \alpha_{2}}\right), \quad\left[q_{3}, q_{2}\right]=\alpha_{3}\left(\alpha_{3}+\alpha_{4}\right)\left(\alpha_{1} \alpha_{2}-\alpha_{4}^{2}\right),} \\
& {\left[p_{2}, q_{2}\right]=-i \rho_{0} \alpha_{2} \alpha_{3}\left(\alpha_{3}+\alpha_{4}\right) \text {, }} \\
& {\left[p_{3}, q_{3}\right]=i \rho_{0} \alpha_{2} \alpha_{3}\left(\alpha_{3}+\alpha_{4}\right) \rho^{2} \text {, }} \\
& {\left[p_{2}, q_{3}\right]=\left[p_{3}, q_{2}\right]} \\
& =\alpha_{3}\left(\alpha_{3}+\alpha_{4}\right)\left(\sqrt{\alpha_{1} \alpha_{2}}-\alpha_{4}\right) \text {, } \\
& 2\left\{p_{2}, p_{3}\right\}=i \rho_{0}\left(\alpha_{3}+\alpha_{4}\right)\left(\alpha_{3}-\sqrt{\alpha_{1} \alpha_{2}}\right), \\
& \left\{p_{2}, p_{2}\right\}=\left(\alpha_{3}+\alpha_{4}\right)^{2} \\
& \left\{p_{3}, p_{3}\right\}=-\left(\alpha_{3}+\alpha_{4}\right)^{2} \rho^{2} \text {. }
\end{aligned}
$$

As a result, for matrices that appear in (8.13) and (8.13) we obtain the following expressions:

$$
\begin{gathered}
b \Delta b^{-1}=\frac{1}{2\left(\alpha_{3}+\sqrt{\alpha_{1} \alpha_{2}}\right)}\left(\begin{array}{cc}
-i \rho_{0}\left(\alpha_{3}-\sqrt{\alpha_{1} \alpha_{2}}\right) & -2\left(\alpha_{3}+\alpha_{4}\right) \\
-2 \rho^{2}\left(\alpha_{3}+\alpha_{4}\right) & i \rho_{0}\left(\alpha_{3}-\sqrt{\alpha_{1} \alpha_{2}}\right)
\end{array}\right), \\
c b^{-1}=\frac{\alpha_{3}}{\alpha_{3}+\sqrt{\alpha_{1} \alpha_{2}}}\left(\begin{array}{cc}
-i \rho_{0} \alpha_{2} \rho^{2} & -\left(\sqrt{\alpha_{1} \alpha_{2}}-\alpha_{4}\right) \\
\sqrt{\alpha_{1} \alpha_{2}}-\alpha_{4} & -i \rho_{0} \alpha_{2}
\end{array}\right), \\
b c^{-1}=\frac{1}{\alpha_{1} \alpha_{2}-\alpha_{4}^{2}}\left(\begin{array}{cc}
i \rho_{0} \alpha_{2} & -\left(\sqrt{\alpha_{1} \alpha_{2}}-\alpha_{4}\right) \\
\sqrt{\alpha_{1} \alpha_{2}}-\alpha_{4} & i \rho_{0} \alpha_{2} \rho^{2}
\end{array}\right) .
\end{gathered}
$$

Hence after elementary calculations

$$
G_{11}(\xi)=\frac{\rho_{0}}{\alpha_{3}+\sqrt{\alpha_{1} \alpha_{2}}}\left(\begin{array}{cc}
\rho^{2}\left(\alpha_{2} \xi_{1}^{2}+\alpha_{3} \xi_{2}^{2}\right) & \left(\alpha_{3}+\alpha_{4}\right) \xi_{1} \xi_{2} \\
\rho^{2}\left(\alpha_{3}+\alpha_{4}\right) \xi_{1} \xi_{2} & \alpha_{3} \xi_{1}^{2}+\alpha_{1} \xi_{2}^{2}
\end{array}\right),
$$




$$
\begin{gathered}
G_{22}(\xi)=\rho_{0}\left(\begin{array}{cc}
\xi_{1}^{2} & \rho^{2} \xi_{1} \xi_{2} \\
\xi_{1} \xi_{2} & \rho^{2} \xi_{2}^{2}
\end{array}\right), \\
G_{21}(\xi)=\frac{\alpha_{3} \rho_{0}}{\alpha_{3}+\sqrt{\alpha_{1} \alpha_{2}}}\left(\begin{array}{cc}
\rho^{2} \xi_{1} \xi_{2} g_{1}(\xi) & -\rho^{4} \alpha_{2} \widehat{\xi}^{2}-\delta \xi_{1}^{2} \\
\rho^{2}\left[\alpha_{2} \widehat{\xi}^{2}+\delta \xi_{2}^{2}\right] & \xi_{1} \xi_{2} g_{2}(\xi)
\end{array}\right), \\
G_{12}(\xi)=\frac{\rho_{0}}{\alpha_{1} \alpha_{2}-\alpha_{4}^{2}}\left(\begin{array}{cc}
-\xi_{1} \xi_{2} g_{1}(\xi) & \rho^{2}\left[\rho^{2} \alpha_{2} \widehat{\xi}^{2}-\delta \xi_{2}^{2}\right] \\
-\rho^{2} \alpha_{2} \widehat{\xi}^{2}+\delta \xi_{1}^{2} & -\rho^{2} \xi_{1} \xi_{2} g_{2}(\xi)
\end{array}\right),
\end{gathered}
$$

where for brevity $\delta=\sqrt{\alpha_{1} \alpha_{2}}-\alpha_{4}, \widehat{\xi}^{2}=\xi_{1}^{2}-\rho^{2} \xi_{2}^{2}$ and we put

$$
g_{j}(\xi)=\alpha_{2}\left(\rho^{2}+1\right) \widehat{\xi}^{2}|\xi|^{-2}+(-1)^{j}\left[\alpha_{2} \rho_{0}^{2} \xi_{j}^{2}|\xi|^{-2}-\delta\right], j=1,2 .
$$

These formulas are even simpler in the case of an isotropic medium, where relations (1.26): $\alpha_{1}=$ $\alpha_{2}=2 \alpha_{3}+\alpha_{4}$ or, equivalently, $\rho=1, \rho_{0}=2$. In particular, $\delta=2 \alpha_{3}, g_{j}(\xi)=2(-1)^{j}\left(\alpha_{1}-\alpha_{3}\right)$, $\widehat{\xi}^{2}=\xi_{1}^{2}-\xi_{2}^{2}$. In this case we have the multiple root $\nu=i$ and the inequality $\alpha_{1}>\alpha_{3}$. In terms of the positive constant $\varkappa=\left(\alpha_{1}+\alpha_{3}\right) /\left(\alpha_{1}-\alpha_{3}\right)$ this yields

$$
\begin{gathered}
c b^{-1}=\frac{\alpha_{3}}{\varkappa}\left(\begin{array}{cc}
-i(\varkappa+1) & -(\varkappa-1) \\
\varkappa-1 & -i(\varkappa+1)
\end{array}\right), \quad b c^{-1}=\frac{1}{4 \alpha_{3}}\left(\begin{array}{cc}
i(\varkappa+1) & -(\varkappa-1) \\
\varkappa-1 & i(\varkappa+1)
\end{array}\right), \\
G_{11}(\xi)=|\xi|^{2}+\frac{1}{\varkappa} G_{1}(\xi), \quad G_{22}(\xi)=|\xi|^{2}+G_{1}(\xi), \\
G_{21}(\xi)=\frac{\alpha_{3}}{\varkappa}\left[(\varkappa-1)|\xi|^{2}+2 G_{2}(\xi)\right] E, \quad G_{12}(\xi)=\frac{1}{4 \alpha_{3}}\left[(\varkappa-1)|\xi|^{2}-2 G_{2}(\xi)\right] E,
\end{gathered}
$$

where we put

$$
G_{1}(\xi)=\left(\begin{array}{cc}
\xi_{1}^{2}-\xi_{2}^{2} & 2 \xi_{1} \xi_{2} \\
2 \xi_{1} \xi_{2} & \xi_{2}^{2}-\xi_{1}^{2}
\end{array}\right), G_{2}(\xi)=\left(\begin{array}{cc}
\varkappa\left(\xi_{1}^{2}-\xi_{2}^{2}\right) & -2 \xi_{1} \xi_{2} \\
-2 \xi_{1} \xi_{2} & \xi_{1}^{2}-\xi_{2}^{2}
\end{array}\right), E=\left(\begin{array}{cc}
0 & -1 \\
1 & 0
\end{array}\right) .
$$

Thus according to definitions (6.5), (6.6), and (8.2) the operators $P_{k r}(k, r=1,2)$ can be written in the form

$$
\begin{gathered}
P_{11}=P+\frac{1}{\varkappa} P_{1}, \quad P_{22}=P+P_{1}, \\
P_{21}=\frac{\alpha_{3}}{\varkappa}\left[(\varkappa-1) P+2 P_{2}\right] E, \quad P_{12}=\frac{1}{4 \alpha_{3}}\left[(\varkappa-1) P-2 P_{2}\right] E,
\end{gathered}
$$

where

$$
\left.P_{j} \varphi\right)(z)=\frac{1}{\pi} \int_{\Gamma} \frac{\operatorname{Re}[\overline{n(t)}(t-z)]}{|t-z|^{4}} G_{j}(t-z) \varphi(t)|d t| .
$$

Respectively, according to (6.11) each pair of equalities

$$
\begin{aligned}
& u=P \varphi+\frac{1}{\varkappa} P_{1} \varphi, \quad v=\frac{\alpha_{3}}{\varkappa}(\varkappa+1) Q \varphi+\frac{\alpha_{3}}{\varkappa}\left[(\varkappa-1) P+2 P_{2}\right] E \varphi, \\
& u=\frac{1}{4 \alpha_{3}}(\varkappa+1) Q \varphi+\frac{1}{4 \alpha_{3}}\left[(\varkappa-1) P-2 P_{2}\right] E \varphi, \quad v=P \varphi+P_{1} \varphi
\end{aligned}
$$

defines the solution $u$ of the Lamé system and its conjugate function $v$. 


\section{Dirichlet Problem in a Piecewise Homogeneous Medium}

We illustrate the above results on the Dirichlet problem for the Lamé system with piecewise constant coefficients. Let a bounded simply connected domain $D$ in the plain have a smooth contour $\Gamma_{1} \in C^{1, \nu}$, $0<\nu<1$, as its boundary and contain a simple smooth contour $\Gamma_{0}$ of the same class that divides this domain into a simply connected subdomain $D^{0}$ and a doubly connected one $D^{1}$. Consider in $D$ the Lamé system (1.1) with piecewise constant coefficients taking constant values $a_{i j}=a_{i j}^{k}, k=0,1$, in the domain $D^{k}$. The sense of notation $\alpha_{j}=\alpha_{j}^{k}$, as well as $u=u^{k}, v=v^{k}$ for the vectors $u, v$ and $\sigma=\sigma^{k}$ for the stress tensor, is similar.

Let $n^{k}=\left(n_{1}^{k}, n_{2}^{k}\right)$ denote the exterior unit normal vector on $\partial D^{k}$ (with respect to the domain $D^{k}$ ), in particular, on the contour $\Gamma_{0}=\partial D^{0} \cap \partial D^{1}$ the vectors $n^{0}$ and $n^{1}$ are opposite. The solution $u$ of system (1.1) in the domain $D$ will be understood as the function $u$ such that its restriction $u^{k}$ belongs to the class $C^{1}\left(D^{k} \cap \Gamma_{0}\right), \quad k=0,1$, and is a classical solution of (1.1) in each domain $D^{k}$, and on $\Gamma_{0}$ it satisfies the contact conditions

$$
\left.\left(u^{0}-u^{1}\right)\right|_{\Gamma_{0}}=0,\left.\quad\left(\sigma^{0} n^{0}+\sigma^{1} n^{1}\right)\right|_{\Gamma_{0}}=0 .
$$

In particular, the function $u$ is continuous in the domain $D$.

The contact conditions mean that under an additional assumption of integrability of the partial derivatives $\partial u / \partial x_{j}$, where $x_{1}=x, x_{2}=y$, the function $u$ satisfies the identity

$$
\sum_{i, j=1}^{2} \int_{D} a_{i j} \frac{\partial u}{\partial x_{j}} \frac{\partial \varphi}{\partial x_{i}} d x=0, \quad \varphi \in C_{0}^{\infty}(D) .
$$

In other words, the function $u$ is a generalized solution of system (1.1) in the domain $D$ written in the divergence form

$$
\sum_{i, j=1}^{2} \frac{\partial}{\partial x_{i}}\left(a_{i j} \frac{\partial u}{\partial x_{j}}\right)=0 .
$$

This fact immediately follows from (2.13), (9.1) by means of partial integration.

In terms of the conjugate function $v$ the second contact condition in (9.1) can be integrated. Let $\left(v^{k}\right)_{s}^{\prime}$ denote the derivative on $\Gamma_{0}$ in the clockwise direction. Then, putting $n=n^{0}=-n^{1}$ on $\Gamma_{0}$, by $(2.13),(2.14)$ we have the relation $\sigma^{k} n=\left(v^{k}\right)_{s}^{\prime}$, and as a result (9.1) becomes

$$
\left.\left(u^{0}-u^{1}\right)\right|_{\Gamma_{0}}=0,\left.\quad\left(v^{0}-v^{1}\right)\right|_{\Gamma_{0}}=\xi
$$

with some constant $\xi \in \mathbb{R}^{2}$.

Consider the Dirichlet problem

$$
\left.u\right|_{\Gamma_{1}}=f
$$

in the domain $D$ for the solution $u \in C(\bar{D})$ of system (1.1) in the above sense.

It is well known [6] that this problem is uniquely solvable in the Sobolev class $W_{1}^{2}(D)$. The uniqueness of the solution is proven directly. In fact, under the assumption $u \in C^{1}\left(\overline{D^{k}}\right)$ by Green's Formula we have

$$
\sum_{i, j=1}^{2} \int_{D^{k}} a_{i j} \frac{\partial u}{\partial x_{j}} \frac{\partial u}{\partial x_{i}} d x=\sum_{i, j=1}^{2} \int_{\partial D^{k}}\left(a_{i j} \frac{\partial u}{\partial x_{j}} n_{i}^{k}\right) u d s .
$$

If $u$ satisfies the homogeneous boundary condition (9.3), then the integral over $\Gamma_{1}$ in this equality for $k=1$ disappears, and after summation of these equalities due to (2.13), (9.1) we obtain the relation

$$
\sum_{i, j=1}^{2} \int_{D} a_{i j} \frac{\partial u}{\partial x_{j}} \frac{\partial u}{\partial x_{i}} d x=0
$$


Therefore, in each domain $D^{k}$ the function $u^{k}$ is a trivial solution, i.e., there exist such constants $c_{j}^{k}, k=0,1, j=1,2,3$, that

$$
u_{1}^{k}(x, y)=c_{3}^{k} y+c_{1}^{k}, \quad u_{2}^{k}(x, y)=-c_{3}^{k} x+c_{2}^{k} .
$$

Substituting these expressions into (9.2) and into the homogeneous boundary condition (9.3), we get $c_{j}^{k}=0$.

Note that in the doubly connected domain $D^{1}$ the conjugate function $v^{1}$ is, generally speaking, multivalued and can get a nonzero increment while tracing $\Gamma_{0}$. On the other hand, in a simply connected domain $D^{0}$ the function $v^{0}$ is always single-valued. Therefore under contact conditions (9.2) the function $v^{1}$ will be single-valued as well.

The main purpose of this section consists in equivalent reduction of problem (1.1), (9.3) to a system of Fredholm integral equations on $\Gamma_{0} \cup \Gamma_{1}$, which will be uniquely solvable due to the uniqueness theorem.

Note that matrix (2.19) is invertible in each domain $D^{k}$. This fact can be extended to the case of two pairs of matrices $b$ and $c$.

Lemma 9.1. Let matrices $\alpha^{k}, k=0,1$, of form (1.2) be positive definite, and let matrices $b^{k}, c^{k}$ correspond to $\alpha^{k}$. Then we have

$$
\operatorname{det}\left(\begin{array}{cc}
b^{0} & \overline{b^{1}} \\
c^{0} & \overline{c^{1}}
\end{array}\right) \neq 0
$$

Proof. Suppose the contrary. Then there exists a nonzero vector $\eta=\left(\eta^{0}, \overline{\eta^{1}}\right) \in \mathbb{C}^{4}$ such that

$$
b^{0} \eta^{0}+\overline{b^{1} \eta^{1}}=0, \quad c^{0} \eta^{0}+\overline{c^{1} \eta^{1}}=0 .
$$

In particular,

$$
\operatorname{Re}\left(b^{0} \frac{\eta^{0}}{t+i}+b^{1} \frac{\eta^{1}}{t-i}\right)=\operatorname{Re}\left(c^{0} \frac{\eta^{0}}{t+i}+c^{1} \frac{\eta^{1}}{t-i}\right)=0, \quad t \in \mathbb{R} .
$$

Let $G^{0}$ and $G^{1}$ denote the upper and lower half-planes, respectively. Consider in $G=G^{0} \cup G^{1}$ the analytic vector function

$$
\psi(z)= \begin{cases}\eta^{0}(z+i)^{-1}, & z \in D^{0} \\ \eta^{1}(z-i)^{-1}, & z \in D^{1}\end{cases}
$$

in terms of which the previous equality becomes

$$
\left.\operatorname{Re}\left(b^{0} \psi^{0}+b^{1} \psi^{1}\right)\right|_{\mathbb{R}}=\left.\operatorname{Re}\left(b^{0} \psi^{0}+b^{1} \psi^{1}\right)\right|_{\mathbb{R}}=0 .
$$

Let $\nu_{j}^{k}, j=1,2$, be the roots of the characteristic equation $p_{1}^{k} p_{2}^{k}-\left(p_{3}^{k}\right)^{2}=0$ that correspond to the matrix $\alpha^{k}$. Consider $J^{k}$-analytic functions $\phi^{k}(x, y)$ in the domain $D^{k}$ related to $\psi^{k}$ by $(2.32)$ and (2.33). Here we take into account the fact that for $\operatorname{Im} \nu>0$ the transform $x+i y \rightarrow x+\nu y$ takes the half-plane $D^{k}$ to itself and leaves fixed its boundary points $x \in \mathbb{R}$. Here $\phi^{k}(x)=\psi^{k}(x)$. Therefore, the relation (9.6) also holds for the functions $\phi^{k}$. But then for the function $u=\operatorname{Re} b \varphi$ satisfying the Lamé system in the domain $D^{k}$ with the elasticity matrix $\alpha^{k}$ and for its conjugate function $v=\operatorname{Re} c \phi$, the following contact relations hold:

$$
\left.\left(u^{0}+u^{1}\right)\right|_{\mathbb{R}}=\left.\left(v^{0}+v^{1}\right)\right|_{\mathbb{R}}=0
$$

on the axis $\mathbb{R}$. As above, hence we conclude that equality (9.4) holds for the function $u$ on the whole plane $D=\mathbb{C}$. In its turn, this is possible only for $u=0$, which leads to a contradiction. This proves assertion (9.5).

Consider the formulation of the Dirichlet problem (9.2), (9.3). Denote by a superscript $k$ the operators $P, Q$ and $P_{i j}$ from Section 6 with respect to the domain $D^{k}, k=0,1$, to the exterior normal $n^{k}$ on $\partial D^{k}$ and to the elasticity matrix $\alpha^{k}$. The notation $P^{k *}, Q^{k *}$ and $P_{i j}^{k *}$ for the boundary operators has a similar sense. According to Theorem 7.1 any solutions $u^{k} \in C\left(\overline{D^{k}}\right)$ of the Lamé system in the 
domain $D^{k}$ (such that for $k=1$ the conjugate function $v^{1}$ is single-valued) are representable in the form

$$
u^{0}=P_{11}^{0} \varphi^{0}, \quad u^{1}=P_{11}^{1} \varphi^{1}
$$

with some vector functions $\varphi^{0} \in C\left(\Gamma_{0}\right)$ and $\varphi^{1} \in C\left(\Gamma_{0} \cup \Gamma_{1}\right)$ so that $u^{0}=u^{1}=0$ in these representations implies

$$
\varphi^{0}=\left.\varphi^{1}\right|_{\Gamma_{1}}=0,\left.\quad \varphi^{1}\right|_{\Gamma_{0}} \in \mathbb{R}^{2} .
$$

For the sake of brevity, put $d=c b^{-1}$. Then by (6.11) we have

$$
v^{k}=-\operatorname{Im} d^{k} Q^{k} \varphi^{k}+P_{21}^{k} \varphi^{k}, \quad k=0,1 .
$$

Therefore due to Lemma 6.2 boundary conditions (9.2), (9.3) are reduced to an equivalent system of equations with respect to $\left(\varphi^{0}, \varphi^{1}, \xi\right)$ defined by the equalities

$$
\begin{gathered}
\varphi^{0}-\varphi^{1}+P_{11}^{0 *} \varphi^{0}-P_{11}^{1 *} \varphi^{1}=0 \quad \text { on } \Gamma_{0}, \\
-\left(\operatorname{Im} d^{0}\right) Q^{0 *} \varphi^{0}+\left(\operatorname{Im} d^{1}\right) Q^{1 *} \varphi^{1}+\left(\operatorname{Re} d^{0}\right) \varphi^{0}-\left(\operatorname{Re} d^{1}\right) \varphi^{1} \\
+P_{21}^{0 *} \varphi^{0}-P_{21}^{1 *} \varphi^{1}=\xi \quad \text { on } \Gamma_{0}, \\
\varphi^{1}+P_{11}^{1 *} \varphi^{1}=f \quad \text { on } \Gamma_{1} .
\end{gathered}
$$

From the viewpoint of the solvability it is convenient to consider instead of this system the one with respect to the pair $\left(\varphi^{0}, \varphi^{1}\right)$ only, which is defined by the equalities

$$
\begin{gathered}
\varphi^{0}-\varphi^{1}+P_{11}^{0 *} \varphi^{0}-P_{11}^{1 *} \varphi^{1}=0 \quad \text { on } \Gamma_{0} \\
-\left(\operatorname{Im} d^{0}\right) Q^{0 *} \varphi^{0}+\left(\operatorname{Im} d^{1}\right) Q^{1 *} \varphi^{1}+\left(\operatorname{Re} d^{0}\right) \varphi^{0}-\left(\operatorname{Re} d^{1}\right) \varphi^{1} \\
+P_{21}^{0 *} \varphi^{0}-P_{21}^{1 *} \varphi^{1}+\sum_{j=1,2} l_{j} \int_{\Gamma_{0}} \varphi^{1}(t) l_{j}|d t|=0 \quad \text { on } \Gamma_{0} \\
\varphi^{1}+P_{11}^{1 *} \varphi^{1}=f \quad \text { on } \quad \Gamma_{1},
\end{gathered}
$$

where $\varphi^{1}(t) l_{j}$ denotes the scalar product in $\mathbb{R}^{2}$ with basic elements $l_{1}=(1,0)$ and $l_{2}=(0,1)$.

Lemma 9.2. The system of equations (9.11) has only the zero solution in the class $\varphi^{0} \in C^{1, \mu}\left(\Gamma_{0}\right)$ and $\varphi^{1} \in C^{1, \mu}\left(\Gamma_{0} \cup \Gamma_{1}\right), \quad 0<\mu<\nu$.

Proof. The functions $\varphi^{0} \in C^{1, \mu}\left(\Gamma_{0}\right)$ and $\varphi^{1} \in C^{1, \mu}\left(\Gamma_{0} \cup \Gamma_{1}\right), \quad 0<\mu<\nu$, satisfy system (9.10) with $f=0$ if and only if the relations (9.8) and $\xi=0$ hold.

In fact, by Lemma 6.2 functions $u^{k}=P_{11}^{k} \varphi^{k}$ belong to $C^{1, \mu}\left(\overline{D^{k}}\right)$ and define the solution of the homogeneous Dirichlet problem in the domain $D$. Therefore, as it was shown above, these function equal zero. Therefore by Theorem 7.1 relations (9.8) hold. Due to Lemmas 7.1, 7.2 we also conclude hence that $v^{1}=P_{21} \varphi^{1}=0$. Therefore the constant $\xi$ in (9.10) also equals zero. The converse statement is obvious, since (9.8) implies $P_{11}^{k} \varphi^{k}=0$.

Now let the pair $\left(\varphi^{0}, \varphi^{1}\right)$ be a solution of the homogeneous system (9.11). Then by the previous proposition relations (9.8) and $\eta l_{1}=\eta l_{2}=0$ hold, where $\eta \in \mathbb{R}^{2}$ denotes the restriction of $\varphi^{1}$ to $\Gamma_{0}$. Consequently, $\varphi^{0}=\varphi^{1}=0$.

For definiteness, put $n=n^{0}=-n^{1}$ on $\Gamma_{0}$, and for the sake of brevity,

$$
p(n, \xi)=|\xi|^{-2} \operatorname{Re}(\bar{n} \xi), q(n, \xi)=|\xi|^{-2} \operatorname{Im}(\bar{n} \xi), \quad n, \xi \in \mathbb{C} .
$$


Then due to (6.1)-(6.3) system (9.11) can be represented in our notation in the following explicit form:

$$
\begin{gathered}
\varphi^{0}\left(t_{0}\right)-\varphi^{1}\left(t_{0}\right)+\frac{1}{\pi} \int_{\Gamma_{0}} p\left[n(t), t-t_{0}\right] \sum_{k=0,1} H_{11}^{k}\left(t-t_{0}\right) \varphi^{k}(t)|d t|- \\
-\frac{1}{\pi} \int_{\Gamma_{1}} p\left[n^{1}(t), t-t_{0}\right]\left[H_{11}^{1}\left(t-t_{0}\right) \varphi^{1}(t)|d t|=0, \quad t_{0} \in \Gamma_{0},\right. \\
\operatorname{Re}\left[d^{0} \varphi^{0}\left(t_{0}\right)-d^{1} \varphi^{1}\left(t_{0}\right)\right]-\frac{1}{\pi} \int_{\Gamma_{0}} q\left[n(t), t-t_{0}\right] \operatorname{Im}\left[d^{0} \varphi^{0}(t)+d^{1} \varphi^{1}(t)\right]|d t|+ \\
+\frac{1}{\pi} \int_{\Gamma_{0}} p\left[n(t), t-t_{0}\right] \sum_{k=0,1} H_{21}^{k}\left(t-t_{0}\right) \varphi^{k}(t)|d t|- \\
-\frac{1}{\pi} \int_{\Gamma_{1}}\left[p\left(n^{1}(t), t-t_{0}\right) H_{21}^{1}\left(t-t_{0}\right)-q\left(n^{1}(t), t-t_{0}\right)\right]\left(\operatorname{Im} d^{1}\right) \varphi^{1}(t)|d t|+ \\
+l_{1} \int_{\Gamma_{0}} \varphi^{1}(t) l_{1}|d t|+l_{2} \int_{\Gamma_{0}} \varphi^{1}(t) l_{2}|d t|=0, \quad t_{0} \in \Gamma_{0}, \\
\varphi^{1}\left(t_{0}\right)+\frac{1}{\pi} \int_{\Gamma_{1}} p\left[n^{1}(t), t-t_{0}\right] H_{11}^{1}\left(t-t_{0}\right) \varphi^{1}(t)|d t|- \\
-\frac{1}{\pi} \int_{\Gamma_{0}} p\left[n(t), t-t_{0}\right]\left[H_{11}^{1}\left(t-t_{0}\right) \varphi^{1}(t)|d t|=f\left(t_{0}\right), \quad t_{0} \in \Gamma_{1} .\right.
\end{gathered}
$$

This system can be briefly rewritten in the operator form. For this purpose, we put

$$
\varphi_{0}=\left.\left(\varphi^{0}, \varphi^{1}\right)\right|_{\Gamma_{0}}, \quad \varphi_{1}=\left.\varphi^{1}\right|_{\Gamma_{1}},
$$

and let operators $P_{0}^{*}$ and $Q_{0}^{*}$ be defined as in (6.1), (6.2) with respect to $\Gamma_{0}$, in the space of vector functions $\varphi_{0}$ they act component-wise. Thus similarly to (6.3) we can write

$$
P_{0}^{*}-i Q_{0}^{*}=S_{0}
$$

with the respective Cauchy singular operator $S_{0}$. Further we introduce integral operators $K_{0}, K_{1}$ and $K_{01}, K_{10}$ by the formulas

$$
\begin{aligned}
& \left(K_{0} \varphi_{0}\right)^{0}\left(t_{0}\right)=\frac{1}{\pi} \int_{\Gamma_{0}} p\left[n(t), t-t_{0}\right] \sum_{k=0,1} H_{11}^{k}\left(t-t_{0}\right) \varphi_{0}^{k}(t)|d t|, \\
& \left(K_{0} \varphi_{0}\right)^{1}\left(t_{0}\right)=\frac{1}{\pi} \int_{\Gamma_{0}} p\left[n(t), t-t_{0}\right] \sum_{k=0,1} H_{21}^{k}\left(t-t_{0}\right) \varphi_{0}^{k}(t)|d t|+ \\
& +l_{1} \int_{\Gamma_{0}} \varphi_{0}^{1}(t) l_{1}|d t|+l_{2} \int_{\Gamma_{0}} \varphi_{0}^{1}(t) l_{2}|d t|, \\
& \left(K_{01} \varphi_{1}\right)^{0}\left(t_{0}\right)=\frac{1}{\pi} \int_{\Gamma_{1}} p\left[n^{1}(t), t-t_{0}\right]\left[H_{11}^{1}\left(t-t_{0}\right)-q\left(n^{1}(t), t-t_{0}\right)\right]\left(\operatorname{Im} d^{1}\right) \varphi_{1}(t)|d t|, \\
& \left(K_{01} \varphi_{1}\right)^{1}\left(t_{0}\right)=\frac{1}{\pi} \int_{\Gamma_{1}} p\left[n^{1}(t), t-t_{0}\right] H_{21}^{1}\left(t-t_{0}\right) \varphi_{1}(t)|d t|, \quad t_{0} \in \Gamma_{0}
\end{aligned}
$$


and

$$
\begin{aligned}
& \left(K_{1} \varphi_{1}\right)\left(t_{0}\right)=\frac{1}{\pi} \int_{\Gamma_{1}} p\left[n^{1}(t), t-t_{0}\right] H_{11}^{1}\left(t-t_{0}\right) \varphi_{1}(t)|d t|, \\
& \left(K_{10} \varphi_{0}\right)\left(t_{0}\right)=\frac{1}{\pi} \int_{\Gamma_{0}} p\left[n(t), t-t_{0}\right]\left[H_{11}^{1}\left(t-t_{0}\right) \varphi_{0}^{1}(t)|d t|, \quad t_{0} \in \Gamma_{1} .\right.
\end{aligned}
$$

In this notation system (9.11) will take the form

$$
(\operatorname{Re} D) \varphi_{0}-(\operatorname{Im} D) Q_{0}^{*} \varphi_{0}+K_{0} \varphi_{0}-K_{01} \varphi_{1}=0, \quad \varphi_{1}+K_{1} \varphi_{1}-K_{10} \varphi_{0}=f
$$

with the matrix

$$
D=\left(\begin{array}{cc}
1 & -1 \\
d^{0} & -\overline{d^{1}}
\end{array}\right)=\left(\begin{array}{cc}
b^{0} & \overline{b^{1}} \\
c^{0} & \overline{c^{1}}
\end{array}\right)\left(\begin{array}{cc}
\left(b^{0}\right)^{-1} & 0 \\
0 & -\left(\overline{b^{1}}\right)^{-1}
\end{array}\right),
$$

which by Lemma 9.1 is invertible.

According to [13], the operator $S_{0}$ in $(9.12)$ possesses the property $S_{0}^{2}=1$. Since it commutes with the operator of multiplication by constant matrices, we conclude hence that the operator

$$
(\operatorname{Re} D) \varphi_{0}+i(\operatorname{Im} D) S_{0}=\left[D\left(1+S_{0}\right)+\bar{D}\left(1-S_{0}\right)\right] / 2
$$

is invertible, and its inverse is

$$
\left(\operatorname{Re} D^{-1}\right) \varphi_{0}+i\left(\operatorname{Im} D^{-1}\right) S_{0}=\left[D^{-1}\left(1+S_{0}\right)+\bar{D}^{-1}\left(1-S_{0}\right)\right] / 2 .
$$

Therefore, writing the first equation of system (9.15) in the form

$$
\left[(\operatorname{Re} D)+i(\operatorname{Im} D) S_{0}-i(\operatorname{Im} D) P_{0}^{*}+K_{0}\right] \varphi_{0}-K_{01} \varphi_{1}-\xi_{0}=0,
$$

we obtain

$$
\varphi_{0}+\left[\left(\operatorname{Re} D^{-1}\right)+i\left(\operatorname{Im} D^{-1}\right) S_{0}\right]\left[-i(\operatorname{Im} D) P_{0}^{*} \varphi_{0}+K_{0} \varphi_{0}-K_{01} \varphi_{1}\right]=0,
$$

or, after separating the real part,

$$
\varphi_{0}+\left[\left(\operatorname{Re} D^{-1}\right)-\left(\operatorname{Im} D^{-1}\right) Q_{0}^{*}\right]\left[K_{0} \varphi_{0}-K_{01} \varphi_{1}\right]+\left(\operatorname{Im} D^{-1}\right)(\operatorname{Im} D)\left(P_{0}^{*}\right)^{2} \varphi_{0}=0 .
$$

As a result, we arrive to the system of Fredholm equations equivalent to (9.14)

$$
\varphi_{0}+\widetilde{K}_{0} \varphi_{0}-\widetilde{K}_{01} \varphi_{1}=0, \quad \varphi_{1}+K_{1} \varphi_{1}-K_{10} \varphi_{0}=f
$$

with the operators

$$
\begin{aligned}
& \widetilde{K}_{0}=\left[\left(\operatorname{Re} D^{-1}\right)-\left(\operatorname{Im} D^{-1}\right) Q_{0}^{*}\right] K_{0}+\left(\operatorname{Im} D^{-1}\right)(\operatorname{Im} D)\left(P_{0}^{*}\right)^{2}, \\
& \widetilde{K}_{01}=\left[\left(\operatorname{Re} D^{-1}\right)-\left(\operatorname{Im} D^{-1}\right) Q_{0}^{*}\right] K_{01} .
\end{aligned}
$$

\section{Regularity of Matrix Kernels of Integral Operators}

Consider in more detail the structure of matrix kernels of operators in (9.16) including the dependence of their regularity upon that of the contours $\Gamma_{j}$. For this purpose, write the operators $P_{0}$ and $S_{0}$ in the complex form, setting

$$
\begin{array}{ll}
\left(P_{0}^{*} \varphi_{0}\right)\left(t_{0}\right)=\frac{1}{\pi i} \int_{\Gamma_{0}} \frac{p_{0}^{*}\left(t_{0}, t\right)}{t-t_{0}} \varphi_{0}(t) d t, & p_{0}^{*}\left(t_{0}, t\right)=\frac{\operatorname{Im}\left[n(t)\left(\bar{t}-\bar{t}_{0}\right)\right]}{n(t)\left(\bar{t}-\bar{t}_{0}\right)} \\
\left(Q_{0}^{*} \varphi_{0}\right)\left(t_{0}\right)=\frac{1}{\pi i} \int_{\Gamma_{0}} \frac{q_{0}^{*}\left(t_{0}, t\right)}{t-t_{0}} \varphi_{0}(t) d t, & q_{0}^{*}\left(t_{0}, t\right)=\frac{\operatorname{Im}\left[n(t)\left(\bar{t}-\bar{t}_{0}\right)\right]}{n(t)\left(\bar{t}-\bar{t}_{0}\right)}
\end{array}
$$


where $d t=i\left(n_{1}+i n_{2}\right)|d t|$ is the complex differential corresponding to anti-clockwise orientation of the contour, and we set $n=n_{1}+i n_{2}, \xi=\xi_{1}+i \xi_{2}$. We treat operators (9.13) similarly:

$$
\begin{aligned}
& \left(K_{0} \varphi_{0}\right)\left(t_{0}\right)=\frac{1}{\pi i} \int_{\Gamma_{0}} \frac{k_{0}\left(t_{0}, t\right)}{t-t_{0}} \varphi_{0}(t) d t, \quad k_{0}\left(t_{0}, t\right)= \\
& =\frac{\operatorname{Re}\left[n(t)\left(\bar{t}-\bar{t}_{0}\right)\right]}{n(t)\left(\bar{t}-\bar{t}_{0}\right)}\left(\begin{array}{ll}
H_{11}^{0}\left(t-t_{0}\right) & H_{11}^{1}\left(t-t_{0}\right) \\
H_{21}^{0}\left(t-t_{0}\right) & H_{21}^{1}\left(t-t_{0}\right)
\end{array}\right)+\frac{t-t_{0}}{n(t)}\left(\begin{array}{cc}
0 & 0 \\
E_{1} & E_{2}
\end{array}\right), \\
& \left(K_{1} \varphi_{1}\right)\left(t_{0}\right)=\frac{1}{\pi i} \int_{\Gamma_{1}} \frac{k_{1}\left(t_{0}, t\right)}{t-t_{0}} \varphi_{1}(t) d t \\
& k_{1}\left(t_{0}, t\right)=\frac{\operatorname{Re}\left[n(t)\left(\bar{t}-\bar{t}_{0}\right)\right]}{n(t)\left(\bar{t}-\bar{t}_{0}\right)} H_{11}^{1}\left(t-t_{0}\right), \quad t_{0}, t \in \Gamma_{1},
\end{aligned}
$$

where the matrices $E_{j} \in \mathbb{R}^{2 \times 2}$ are defined by equalities

$$
E_{1}=\left(\begin{array}{ll}
1 & 0 \\
0 & 1
\end{array}\right), \quad E_{2}=\left(\begin{array}{cc}
0 & 1 \\
1 & 0
\end{array}\right)
$$

and

$$
\begin{gathered}
\left(K_{01} \varphi_{1}\right)\left(t_{0}\right)=\frac{1}{\pi i} \int_{\Gamma_{1}} \frac{k_{01}\left(t_{0}, t\right)}{t-t_{0}} \varphi_{1}(t) d t, \\
k_{01}\left(t_{0}, t\right)=\frac{\operatorname{Re}\left[n(t)\left(\bar{t}-\bar{t}_{0}\right)\right]}{n(t)\left(\bar{t}-\bar{t}_{0}\right)}\left(\begin{array}{c}
H_{11}^{1}\left(t-t_{0}\right) \\
H_{21}^{1}\left(t-t_{0}\right)
\end{array}\right)-\frac{\operatorname{Im}\left[n(t)\left(\bar{t}-\bar{t}_{0}\right)\right]}{n(t)\left(\bar{t}-\bar{t}_{0}\right)}\left(\begin{array}{c}
\operatorname{Im} d^{1} \\
0
\end{array}\right), \\
\left(K_{10} \varphi_{0}\right)\left(t_{0}\right)=\frac{1}{\pi i} \int \frac{k_{10}\left(t_{0}, t\right)}{t-t_{0}} \varphi_{0}(t) d t, \\
k_{10}\left(t_{0}, t\right)=\frac{\operatorname{Re}\left[n(t)\left(\bar{t}-\bar{t}_{0}\right)\right]}{n(t)\left(\bar{t}-\bar{t}_{0}\right)} H_{11}^{1}\left(t-t_{0}\right), \quad t_{0} \in \Gamma_{1}, t \in \Gamma_{0} .
\end{gathered}
$$

Further we make use of the following auxiliary result.

Lemma 10.1. Let a function $h(\xi), \xi=\xi_{1}+i \xi_{2}$, be infinitely differentiable for $\xi \neq 0$, homogeneous of order zero and even. Then on the regular contour $\Gamma$ of the class $C^{m, \nu}$, where $m$ is a natural number and $0<\nu<1$, the function $k\left(t_{1}, t\right)=h\left(t_{1}-t\right)$ belongs to the class $C^{m-1, \nu}(\Gamma \times \Gamma)$ and for $t_{1}=t$ takes the value $h[i n(t)]$, where $n(t)=n_{1}(t)+i n_{2}(t)$ is the unit normal to $\Gamma$.

Proof. It suffices to prove the lemma in a neighborhood of a fixed point $\left(t_{0}, t_{0}\right) \in \Gamma \times \Gamma$. Parameterize the contour in this neighborhood in the form $z=z(s),|s| \leq \delta$, where $s$ is the arc length parameter measured from the point $t_{0}$. Then the function

$$
\alpha\left(s_{1}, s\right)=\frac{z(s)-z\left(s_{1}\right)}{s-s_{1}}=\int_{0}^{1} z^{\prime}\left[s \tau+s_{1}(1-\tau)\right] d \tau
$$

belongs to the class $C^{m-1, \nu}$ in the square $\left|s_{1}\right|,|s| \leq \delta$, and is separated from zero in absolute value and takes the value $z^{\prime}(s)$ for $s_{1}=s$. Therefore the function $k\left[z\left(s_{1}\right), z(s)\right]=h\left[\alpha\left(s_{1}, s\right)\right]$ also belongs to this class and its value at $s_{1}=s$ coincides with $h\left[z^{\prime}(s)\right]=h[i n(s)]$. This implies the assertion of the lemma for the function $k\left(t_{1}, t\right)$ in the neighborhood of the contour under consideration.

This Lemma and (10.1)-(10.3) imply that under the hypothesis $\Gamma_{j} \in C^{m, \nu}, m \geq 1$, the functions

$$
p_{0}^{*}, q_{0}^{*}, k_{0} \in C^{m-1, \nu}\left(\Gamma_{0} \times \Gamma_{0}\right), \quad k_{1} \in C^{m-1, \nu}\left(\Gamma_{1} \times \Gamma_{1}\right),
$$


and

$$
p_{0}^{*}(t, t)=0, \quad q_{0}^{*}(t, t)=-i, \quad k_{j}(t, t)=0, j=0,1 .
$$

As for the continuous functions $k_{0,1}\left(t_{0}, t\right)$ and $k_{1,0}\left(t_{0}, t\right)$, as functions of the variable $t_{0}$ they belong respectively to $C^{m, \nu}\left(\Gamma_{0}\right)$ and to $C^{m, \nu}\left(\Gamma_{1}\right)$ uniformly in $t$.

Now consider the operator products $Q_{0}^{*} K_{0}$ and $\left(P_{0}^{*}\right)^{2}$ that appear in expression (9.16) for the operator $\widetilde{K}_{0}$. According to $(10.1)$, (10.2) we have:

$$
\left(Q_{0}^{*} K_{0} \varphi\right)\left(t_{1}\right)=\frac{1}{\pi i} \int_{\Gamma_{0}} \frac{q_{0}^{*}\left(t_{1}, t_{0}\right) d t_{0}}{t_{0}-t_{1}} \frac{1}{\pi i} \int_{\Gamma_{0}} \frac{k_{0}\left(t_{0}, t\right) \varphi(t) d t}{t-t_{0}}, \quad t_{1} \in \Gamma_{0} .
$$

Due to (10.5) it is possible to change the order of the two integrations here, where the first one is singular [13], so that

$$
\left(Q_{0}^{*} K_{0} \varphi\right)\left(t_{1}\right)=\frac{1}{\pi i} \int_{\Gamma_{0}} \varphi(t) d t \frac{1}{\pi i} \int_{\Gamma_{0}} \frac{q_{0}^{*}\left(t_{1}, t_{0}\right) k_{0}\left(t_{0}, t\right) d t_{0}}{\left(t_{0}-t_{1}\right)\left(t-t_{0}\right)} .
$$

Since

this finally yields

$$
\frac{t-t_{1}}{\left(t_{0}-t_{1}\right)\left(t-t_{0}\right)}=\frac{1}{t_{0}-t_{1}}-\frac{1}{t_{0}-t}
$$

$$
\left(Q_{0}^{*} K_{0} \varphi\right)\left(t_{1}\right)=\frac{1}{\pi i} \int_{\Gamma_{0}} \frac{\left[k^{*}\left(t_{1}, t, t_{1}\right)-k^{*}\left(t_{1}, t, t\right)\right] \varphi(t) d t}{t-t_{1}}, \quad t_{1} \in \Gamma_{0},
$$

where

$$
k^{*}\left(t_{1}, t, t_{2}\right)=\frac{1}{\pi i} \int_{\Gamma_{0}} \frac{q_{0}^{*}\left(t_{1}, t_{0}\right) k_{0}\left(t_{0}, t\right) d t_{0}}{t_{0}-t_{2}} .
$$

Lemma 10.2. Let $\Gamma_{0} \in C^{m+1, \nu}$, where $m$ is a nonnegative integer and $0<\nu<1$. Then the function $k^{*}\left(t_{1}, t, t_{2}\right)$ in all three variables belongs to the class $C^{m, \nu-\varepsilon}$ for each $\varepsilon>0$, i.e.,

$$
k^{*}\left(t_{1}, t, t_{2}\right) \in C^{m, \nu-0}\left(\Gamma_{0} \times \Gamma_{0} \times \Gamma_{0}\right) .
$$

Proof. According to (10.4), the function $k\left(t_{1}, t, t_{0}\right)=q^{*}\left(t_{1}, t_{0}\right) k_{0}\left(t_{0}, t\right)$ belongs to the class $C^{m, \nu-0}\left(\Gamma_{0} \times\right.$ $\left.\Gamma_{0} \times \Gamma_{0}\right)$; therefore for $m=0$ the assertion of the Lemma follows from properties [13] of parameterdependent singular integrals.

In the general case $m \geq 1$ we make use of the formula of differentiation of the singular integral $k^{*}$ in all variables. It is convenient to differentiate the function $\varphi$ on $\Gamma_{0}$ in the complex parameter of the contour, i.e., as the limit

$$
\varphi^{\prime}\left(t_{0}\right)=\lim _{t \rightarrow t_{0}, t \in \Gamma_{0}} \frac{\varphi(t)-\varphi\left(t_{0}\right)}{t-t_{0}} .
$$

The partial derivatives are understood similarly. It is well known [13] that a singular integral $p^{*}$ can be differentiated in the parameter under the integration sign:

$$
\frac{\partial k^{*}}{\partial t_{1}}\left(t_{1}, t, t_{2}\right)=\frac{1}{\pi i} \int_{\Gamma_{0}} \frac{\partial k}{\partial t_{1}}\left(t_{1}, t, t_{0}\right) \frac{d t_{0}}{t_{0}-t_{2}}, \quad \frac{\partial k^{*}}{\partial t}\left(t_{1}, t, t_{2}\right)=\frac{1}{\pi i} \int_{\Gamma_{0}} \frac{\partial k}{\partial t}\left(t_{1}, t, t_{0}\right) \frac{d t_{0}}{t_{0}-t_{2}} .
$$

We assert that a similar formula holds in the last variable as well:

$$
\frac{\partial k^{*}}{\partial t_{2}}\left(t_{1}, t, t_{2}\right)=\frac{1}{\pi i} \int_{\Gamma_{0}} \frac{\partial k}{\partial t_{0}}\left(t_{1}, t, t_{0}\right) \frac{d t_{0}}{t_{0}-t_{2}} .
$$


In fact, consider inside the contour $\Gamma_{0}$ the analytic function in the variable $z$

$$
\phi\left(t_{1}, t, z\right)=\frac{1}{\pi i} \int_{\Gamma_{0}} \frac{k\left(t_{1}, t, t_{0}\right) d t_{0}}{t_{0}-z} .
$$

Partial integration shows that

$$
\frac{\partial \phi}{\partial z}\left(t_{1}, t, z\right)=\frac{1}{\pi i} \int_{\Gamma_{0}} \frac{\partial k}{\partial t_{0}}\left(t_{1}, t, t_{0}\right) \frac{d t_{0}}{t_{0}-z} .
$$

By the Sohocki-Plemelj Formula [13] for the boundary values of these functions we have

$$
\begin{gathered}
\phi^{+}\left(t_{1}, t, t_{2}\right)=k\left(t_{1}, t, t_{2}\right)+\frac{1}{\pi i} \int_{\Gamma_{0}} \frac{k\left(t_{1}, t, t_{0}\right) d t_{0}}{t_{0}-t_{2}} \\
\left(\frac{\partial \phi}{\partial z}\right)^{+}\left(t_{1}, t, t_{2}\right)=\frac{\partial k}{\partial t_{2}}\left(t_{1}, t, t_{2}\right)+\frac{1}{\pi i} \int_{\Gamma_{0}} \frac{\partial k}{\partial t_{0}}\left(t_{1}, t, t_{0}\right) \frac{d t_{0}}{t_{0}-t_{2}} .
\end{gathered}
$$

Differentiating the first equality in $t_{0}$ and comparing the result with the second equality, we arrive at the validity of (10.8).

The assertion (10.7) of the lemma follows now directly by induction in $m$ from the differentiation formulas.

In a completely similar way we establish an analogous result for the operator $\left(P_{0}^{*}\right)^{2}$ :

$$
\left[\left(P_{0}^{*}\right)^{2} \varphi\right]\left(t_{1}\right)=\frac{1}{\pi i} \int_{\Gamma_{0}} \frac{\left[k_{0}^{*}\left(t_{1}, t, t_{1}\right)-k_{0}^{*}\left(t_{1}, t, t\right)\right] \varphi(t) d t}{t-t_{1}}, \quad t_{1} \in \Gamma_{0},
$$

where by Lemma 10.2 the function

$$
k_{0}^{*}\left(t_{1}, t, t_{2}\right)=\frac{1}{\pi i} \int_{\Gamma_{0}} \frac{p_{0}^{*}\left(t_{1}, t_{0}\right) p_{0}^{*}\left(t_{0}, t\right) d t_{0}}{t_{0}-t_{2}}
$$

belongs to the class $C^{m, \nu-0}\left(\Gamma_{0} \times \Gamma_{0} \times \Gamma_{0}\right)$.

Therefore by (10.2) and (10.6), (10.9) the operator $\widetilde{K}_{0}$ in (9.16) acts according to the formula

$$
\left(\widetilde{K}_{0} \varphi\right)\left(t_{0}\right)=\frac{1}{\pi i} \int_{\Gamma_{0}} \frac{\widetilde{k}_{0}\left(t_{0}, t\right) \varphi(t) d t}{t-t_{0}}, \quad t_{0} \in \Gamma_{0},
$$

where under the assumption $\Gamma \in C^{m+1, \nu}$ the function

$$
\widetilde{k}_{0}\left(t_{0}, t\right) \in C^{m, \nu-0}\left(\Gamma_{0} \times \Gamma_{0}\right), \quad \widetilde{k}_{0}(t, t)=0 .
$$

The operator $K_{1}$ possesses a similar property with respect to the contour $\Gamma$. Thus (4.10) is a system of Fredholm equations. Before formulating the central result for it, consider the following typical situation related to this system for an integral operator similar to (10.10):

$$
(K \varphi)\left(t_{0}\right)=\frac{1}{\pi i} \int_{\Gamma_{0}} \frac{k\left(t_{0}, t\right) \varphi(t) d t}{t-t_{0}}, \quad t_{0} \in \Gamma .
$$

Lemma 10.3. Under the hypotheses of Lemma 3.1 let the operator $K$ be compact in the space $C^{m, \mu}(\Gamma)$, $m \geq 1$. Then any solution $\varphi \in C(\Gamma)$ of the equation $\varphi+K \varphi=f$ with the right-hand side $f \in C^{m, \mu}(\Gamma)$ also belongs to $f \in C^{m, \mu}(\Gamma)$. 
Proof. According to Lemma 3.1, the operator $K$ is compact in each space $C(\Gamma), C^{\mu}(\Gamma)$, and any solution $\varphi \in C(\Gamma)$ of the equation $\varphi+K \varphi=f$ with the right-hand side $f \in C^{\mu}(\Gamma)$ also belongs to $f \in C^{\mu}(\Gamma)$. In addition, the following Fredholm alternatives hold for this equation:

(1) the homogeneous equation $\varphi+K \varphi=0$ has a finite number $n$ of linearly independent solutions $\varphi_{1}, \ldots, \varphi_{n} \in C(\Gamma)$

(2) the homogeneous associated equation $\psi+K^{\prime} \psi=0$, where the operator $K^{\prime}$ is obtained from (10.10) by changing $k\left(t_{0}, t\right)$ with $-k\left(t, t_{0}\right)$ under the integration sign, has the same number $n$ of linearly independent solutions $\psi_{1}, \ldots, \psi_{n} \in C(\Gamma)$;

(3) the inhomogeneous equation $\varphi+K \varphi=f$ is solvable if and only if

$$
\int_{\Gamma} f(t) \psi_{j}(t)|d t|=0, \quad 1 \leq j \leq n .
$$

Note that in fact the functions $\varphi_{j}$ and $\psi_{j}$ belong to $C^{\mu}(\Gamma)$.

Now let the operator $K$ be compact in $C^{m \mu}(\Gamma)$. Then by the Riesz theorem the Fredholm alternatives also hold for the equation $\varphi+K \varphi=f$ in the class $C^{m, \mu}(\Gamma)$, i.e., the homogeneous equation has $n_{0} \leq n$ linearly independent solutions in this class and there exist such $n_{0}$ linearly independent functionals over $C^{m, \mu}(\Gamma)$ that the fact that they vanish at the function $f$ is necessary and sufficient for the solvability of the inhomogeneous equation $\varphi+K \varphi=f$. But, since conditions (10.13) are necessary for the solvability of this equation in the class $C^{m, \mu}(\Gamma)$ as well, this implies the inequality $n \leq n_{0}$. Thus we have $n_{0}=n$, so that assertions $1-3$ hold with respect to the class $C^{m, \mu}$ as well. In its turn, the second part of the lemma follows immediately.

Theorem 10.1. Let $\Gamma_{0}, \Gamma_{1} \in C^{1, \nu}$. Then system of equations (9.16) is uniquely solvable in the class $\varphi_{0} \in C\left(\Gamma_{0}\right) \varphi_{1} \in C\left(\Gamma_{1}\right)$, and $f \in C^{m, \mu}\left(\Gamma_{1}\right), \quad m=0,1,0<\mu<\nu$, implies $\varphi_{0} \in C^{m, \mu}\left(\Gamma_{0}\right) \varphi_{1} \in$ $C^{m, \mu}\left(\Gamma_{1}\right)$. If in addition $\Gamma_{j} \in C^{m+1, \nu}, m \geq 2$, then the previous assertion also holds for $m \geq 2$.

Proof. According to (10.10), the operator $\widetilde{K}_{0}$ satisfies the hypotheses of the first part of Lemma 10.3, and so does the operator $K_{1}$ on the contour $\Gamma_{1}$. In addition, by Lemma 6.2 these operators are also compact in the space $C^{1, \mu}\left(\Gamma_{1}\right)$. Therefore the first assertion of the Theorem follows from Lemma 10.3 applied to the system of equations on the contours $\Gamma_{j}$ and Lemma 9.1. Suppose further that $\Gamma_{j} \in$ $C^{m+1, \nu}$. Then the function $\widetilde{k}\left(t_{0}, t\right)$ that defines the operator $\widetilde{K}_{0}$ in (10.10) has property $(10.11)$. Therefore the operator $\widetilde{K}_{0}$ is compact in the space $C^{n, \mu}\left(\Gamma_{1}\right)$, which can be easily shown by induction in $m$ with the help of the differentiation formula obtained in the proof of Lemma 10.2. The situation with the operator $K_{1}$ is completely similar, and the claim follows now from Lemma 10.3.

\section{REFERENCES}

1. A. V. Aleksandrov and A. P. Soldatov, "Boundary properties of integrals of the Cauchy type," Differ. Uravn., 27, No. 1, 3-8 (1991).

2. H. Begehr and W. Lin, "A mixed-contact problem in orthotropic elasticity," In: Partial Differential Equations with Real Analysis, 219-239, Longman Scientific \& Technical, Harlow (1992).

3. H. Begehr and W. Lin, "A mixed-contact problem in orthotropic elasticity", In: Complex Analytic Methods for Partial Differential Equations. An Introductory Text, World Scientific, Singapore (1994).

4. A. Douglis, "A function-theoretical approach to elliptic systems of equations in two variables," Commun. Pure Appl. Math., 6, 259-289 (1953).

5. A. H. England, Complex Variable Methods in Elasticity, Wiley-Interscience, London (1971).

6. G. Fikera, Existence Theorems in the Elasticity Theory [in Russian], Mir, Moscow (1974).

7. R. P. Gilbert and W. L. Wendland, "Analytic, generalized, hyper-analytic function theory and an application to elasticity," Proc. Roy. Soc. Edinburgh Sect. A., 73A, 317-371 (1975). 
8. G. M. Goluzin, Geometric Theory of Functions of Complex Argument [in Russian], Nauka, Moscow (1972).

9. I. Ts. Gokhberg and N. I. Krupnik, Introduction to the Theory of One-Dimensional Singular Equations [in Russian], Shtiintsa, Kishinev (1973).

10. V. D. Kupradze, Methods of Potential in the Theory of Elasticity [in Russian], Fizmatgiz, Moscow (1963).

11. G. G. Lekhnitskiy, Elasticity Theory of Anisotropic Body [in Russian], GITTL, MoscowLeningrad (1950).

12. N. I. Muskhelishvili, Some General Problems of the Mathematical Theory of Elasticity [in Russian], Nauka, Moscow (1966).

13. N. I. Muskhelishvili, Singular Integral Equations [in Russian], Nauka, Moscow (1968).

14. R. Pale, Seminar on the Atiyah-Singer Index Theorem [in Russian], Mir, Moscow (1970).

15. U. Rudin, Functional Analysis [in Russian], Mir, Moscow (1991).

16. A. P. Soldatov, "Function theory method for boundary-value problems on the plane. I. Smooth case," Izv. AN SSSR. Ser. Mat., 55, No. 5, 1070-1100 (1991).

17. A. P. Soldatov, "Hyper-analytic functions and their applications," Sovrem. Mat. Prilozh., 15, 142-199 (2004).

18. A. P. Soldatov, "The Hardy space of solutions of first-order elliptic systems," Dokl. Akad. Nauk, 416, No. 1, 26-30 (2007).

19. A. P. Soldatov, "The Dirichlet problem for weakly connected elliptic systems on the plane," Differ. Uravn., 49, No. 6, 734-745 (2013).

20. A. P. Soldatov, "The Neumann problem for elliptic systems on the plane," Sovrem. Mat. Fundam. Napravl., 48, 120-133 (2013).

21. A. P. Soldatov and O. V. Chernova, "The Riemann-Hilbert problem for first-order elliptic system in the Hölder classes," Nauchn. Vedom. BelGU, 13, No. 17/2, 115-121 (2009).

A. P. Soldatov

National Research University "Belgorod State University," Belgorod, Russia

E-mail: soldatov48@gmail.com 